\title{
Direct detection of mirror matter in Twin Higgs models
}

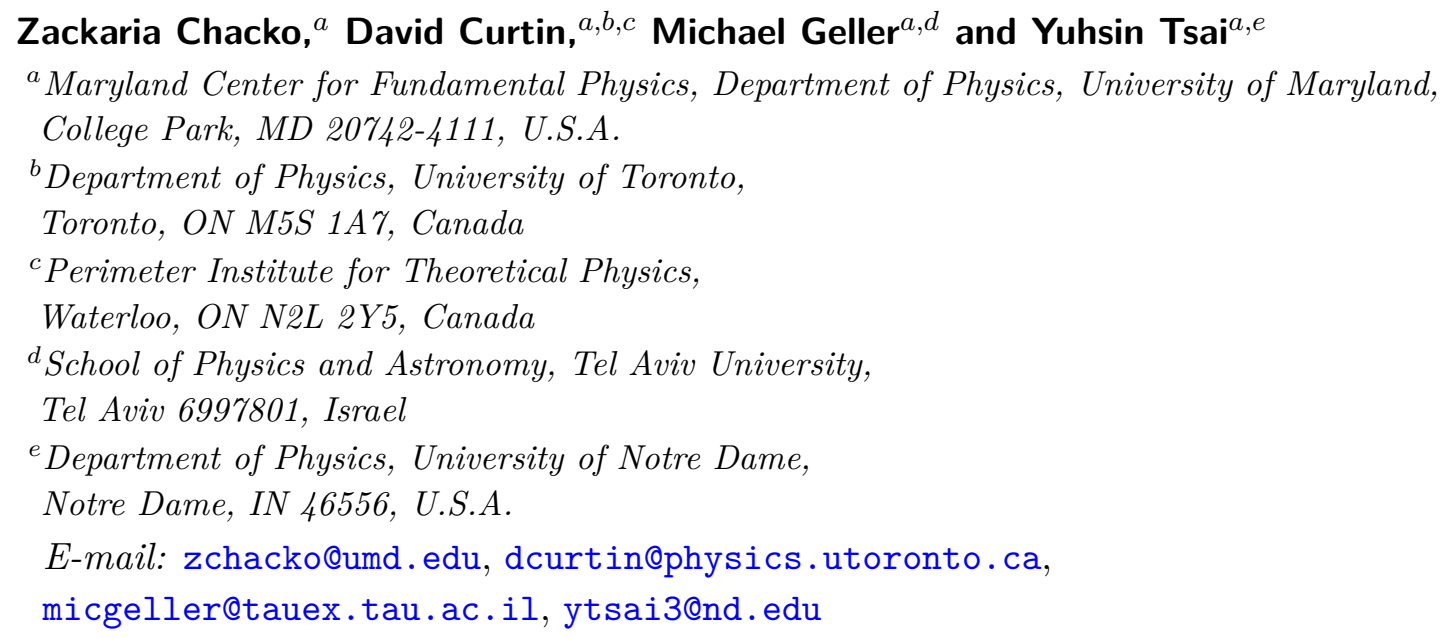

ABSTRACT: We explore the possibility of discovering the mirror baryons and electrons of the Mirror Twin Higgs model in direct detection experiments, in a scenario in which these particles constitute a subcomponent of the observed DM. We consider a framework in which the mirror fermions are sub-nano-charged, as a consequence of kinetic mixing between the photon and its mirror counterpart. We consider both nuclear recoil and electron recoil experiments. The event rates depend on the fraction of mirror DM that is ionized, and also on its distribution in the galaxy. Since mirror DM is dissipative, at the location of the Earth it may be in the form of a halo or may have collapsed into a disk, depending on the cooling rate. For a given mirror DM abundance we determine the expected event rates in direct detection experiments for the limiting cases of an ionized halo, an ionized disk, an atomic halo and an atomic disk. We find that by taking advantage of the complementarity of the different experiments, it may be possible to establish not just the multi-component nature of mirror dark matter, but also its distribution in the galaxy. In addition, a study of the recoil energies may be able to determine the masses and charges of the constituents of the mirror sector. By showing that the mass and charge of mirror helium are integer multiples of those of mirror hydrogen, these experiments have the potential to distinguish the mirror nature of the theory. We also carefully consider mirror plasma screening effects, showing that the capture of mirror dark matter particles in the Earth has at most a modest effect on direct detection signals.

Keywords: Beyond Standard Model, Cosmology of Theories beyond the SM

ArXIV EPRINT: 2104.02074 


\section{Contents}

1 Introduction 1

2 Parameters of the model 4

3 Mirror baryon distribution in the Milky Way 6

$\begin{array}{lll}3.1 & \text { Initial mirror baryon distribution } & 7\end{array}$

$\begin{array}{lll}3.1 .1 & \text { CDM profile } & 7\end{array}$

$\begin{array}{lll}3.1 .2 & \text { Mirror matter in hydrostatic equilibrium } & 8\end{array}$

$\begin{array}{lll}3.1 .3 & \text { Results } & 11\end{array}$

3.1.4 Mirror baryon ionization fractions 14

$\begin{array}{ll}3.2 & \text { Mirror baryon cooling } \\ & 15\end{array}$

$\begin{array}{lll}3.2 .1 & \text { Cooling timescales } & 16\end{array}$

$\begin{array}{lll}3.2 .2 & \text { Optical depth } & 18\end{array}$

$\begin{array}{lll}3.2 .3 & \text { Results } & 20\end{array}$

3.3 Resulting mirror baryon distribution today 21

4 Direct detection of sub-nano-charged mirror matter 24

4.1 Local mirror baryon ionization and velocity distribution 26

$\begin{array}{lll}4.2 & \text { Direct detection via nuclear recoils } & 29\end{array}$

$\begin{array}{lll}4.2 .1 & \text { Review } & 29\end{array}$

4.2.2 Nuclear recoils in the Mirror Twin Higgs 30

4.3 Direct detection via electron recoils 32

$\begin{array}{lll}4.3 .1 & \text { Review } & 32\end{array}$

4.3.2 Electron recoils in the Mirror Twin Higgs 35

$\begin{array}{lll}4.4 & \text { Characterization of the dark sector } & 37\end{array}$

5 Conclusions $\quad 39$

A Capture of mirror matter in the Earth 41

A.1 $\epsilon \lesssim 10^{-11} \quad 42$

A.1.1 Capture of mirror helium $\quad 42$

A.1.2 Capture and evaporation of mirror electrons 46

A.1.3 Screening and the escape velocity 49

$\begin{array}{lll}\text { A.1.4 Evaporation of mirror nuclei } & 55\end{array}$

$\begin{array}{ll}\text { A.1.5 Results } & 57\end{array}$

A.2 $10^{-10} \lesssim \epsilon \lesssim 10^{-9}$

$\begin{array}{lll}\text { A.2.1 Equilibrium in the large charge regime } & 58\end{array}$

$\begin{array}{ll}\text { A.2.2 Upper bound on the net captured charge } & 59\end{array}$

A.2.3 Upper bound on the total number of captured mirror particles $\quad 63$ 


\section{Introduction}

The Mirror Twin Higgs (MTH) framework [1-3] offers a simple and distinctive solution to the little hierarchy problem of the Standard Model (SM). In this class of theories the spectrum of light states includes a complete mirror ("twin") copy of the SM, with the same particle content and gauge groups. A discrete $\mathbb{Z}_{2}$ interchange symmetry relates the particles and interactions in the mirror sector to those in the SM. The Higgs emerges as the pseudoNambu Goldstone boson of an approximate global symmetry which is spontaneously broken. Quadratically divergent corrections to the Higgs mass are cancelled by a combination of the global symmetry and the discrete $\mathbb{Z}_{2}$ symmetry that relates the SM and twin sectors. MTH models stabilize the Higgs mass against radiative corrections up to scales of order 5-10 TeV, above which an ultraviolet completion [4-13] is required.

In the MTH construction, there are no new light states charged under the SM gauge groups. Therefore, this class of models is free from the strong constraints on top partner searches at the Large Hadron Collider (LHC) [14-20]. The only coupling between the SM and mirror sectors that is required by the construction is a Higgs portal interaction between the SM Higgs and its twin partner. Then, after electroweak symmetry breaking in the two sectors, the SM Higgs boson mixes with its twin counterpart. As a result of this mixing, the Higgs acquires couplings to twin fermions and gauge bosons. This allows twin particles to be produced at collider experiments through the Higgs portal. The twin particles are invisible and give rise to missing energy signals at colliders. However, the event rate is low, and missing energy searches at the LHC have only limited sensitivity to twin particle production.

The tightest collider constraints on MTH models are from precision Higgs measurements at the LHC. As a result of the mixing between the SM Higgs and its twin counterpart, the couplings of the Higgs to SM particles are suppressed. In addition, the Higgs acquires couplings to mirror fermions and gauge bosons, and can decay into them. Both these effects contribute to a reduction in the number of Higgs events at the LHC as compared to the SM prediction [21]. The fact that the number of Higgs events observed at the LHC is consistent with the expectation from the SM can be used to place constraints on MTH models. In order to satisfy this constraint, we require a mild hierarchy between the scale of electroweak symmetry breaking in the twin sector, denoted by $\hat{v}$, and the corresponding scale in the SM sector $v$. The constraint is satisfied provided $\hat{v} / v \gtrsim 3[22]$. We can realize this hierarchy by introducing a soft explicit breaking of the discrete $\mathbb{Z}_{2}$ symmetry that relates the two sectors, albeit at the expense of mild tuning. (This $\mathbb{Z}_{2}$ breaking can also occur spontaneously, with a possible reduction of tuning, see e.g. [23-28].) The dynamics of the associated phase transition has been studied in $[29,30]$. A phenomenologically important consequence of the difference in the scales of electroweak symmetry breaking is that the elementary fermions and gauge bosons in the twin sector are heavier by a factor of $\hat{v} / v$ than their SM counterparts.

The MTH framework is severely constrained by cosmology. The Higgs portal interaction keeps the SM and twin sectors in thermal equilibrium until temperatures of order a GeV [2]. Below this temperature, even though the two sectors are decoupled, mirror states continue to contribute almost half of the total energy density in the universe. This results in a large contribution to the energy density in dark radiation during the CMB epoch, 
$\Delta N_{\text {eff }}=5.7[31,32]$. An effect of this size is excluded by the current bounds, which require $\Delta N_{\text {eff }} \lesssim 0.25(2 \sigma)$ from Planck 2018+lensing+BAO, or $\Delta N_{\text {eff }} \lesssim 0.49(2 \sigma)$ [33] if including the $H_{0}$ measurement from ref. [34]. This problem can be solved if there is an additional source of breaking of the discrete $\mathbb{Z}_{2}$ symmetry. This would allow the number of degrees of freedom in the twin sector at the time when the two sectors decouple to be much less than in the SM, leading to a suppression of $\Delta N_{\text {eff }}[2,5,35-41]$. The same result can be achieved by making the mirror sector vector-like [42]. The most radical proposal in this regard is the Fraternal Twin Higgs (FTH) construction [43], in which the first two generations of twin fermions, which do not play a significant role in the solution of the little hierarchy problem, are simply removed from the theory. The FTH framework leads to distinctive collider signatures at the LHC involving displaced vertices [44-47].

An alternative approach to address the problem, which does not require additional breaking of the $\mathbb{Z}_{2}$ symmetry that relates the two sectors, is to introduce into the theory an asymmetric reheating process that preferentially heats up the SM sector [48-50]. The asymmetric reheating should occur at temperatures below $1 \mathrm{GeV}$, after the two sectors have decoupled, but before Big Bang nucleosynthesis (BBN). This reduces the fraction of energy density contained in the twin sector, allowing the bounds on $\Delta N_{\text {eff }}$ to be satisfied [31, 32]. Although the mirror sector contribution to $\Delta N_{\text {eff }}$ is suppressed in this scenario, it is expected to be large enough to be observed in future CMB experiments. Both the MTH and FTH frameworks contain several promising dark matter (DM) candidates [35, 51-61].

If there is a baryon asymmetry in the twin sector, the mirror baryons and electrons will constitute a subcomponent of the DM in the universe. If the discrete $\mathbb{Z}_{2}$ twin symmetry is only softly broken, so that the masses of mirror particles are fixed by the ratio of electroweak VEVs in the two sectors, $\hat{v} / v$, the mirror baryons are primarily composed of mirror hydrogen and helium. The relative abundances of these two species is determined by the dynamics of BBN in the twin sector. Together with the mirror photons and neutrinos, the mirror baryons and electrons can give rise to highly distinctive signals in large scale structure and in the cosmic microwave background [62]. Baryon acoustic oscillations in the mirror sector prior to recombination lead to a suppression of structure on large scales. Current limits on the size of this effect bound the mirror contribution to DM in the MTH framework to be less than $\mathcal{O}(10 \%)$.

Apart from the Higgs portal coupling, the gauge symmetries of the MTH construction allow only one other renormalizable interaction that connects the SM and twin sectors, a kinetic mixing between hypercharge and its twin counterpart,

$$
\frac{\epsilon}{2 \cos \theta_{W}} B_{\mu \nu} B^{\prime \mu \nu}
$$

If this operator is present, the twin fermions acquire a charge under electromagnetism proportional to $\epsilon$. Avoiding thermalization of the hidden and visible sector after asymmetric reheating constrains any such mixing to be very small, less than or of order $10^{-9}$ [63]. However, even such small values of the mixing are radiatively stable in the minimal MTH construction, since this mixing is not generated through 3-loop order [1]. If there is a baryon asymmetry in the twin sector, the mirror fermions will therefore constitute sub-nano-charged 
DM and can scatter off ordinary matter through processes involving the exchange of the photon. Interestingly, it has recently been shown that higher-order loop diagrams involving gravitons could generate a kinetic mixing between the visible and hidden sectors [64]. This contribution, which primarily arises from energies of order the Planck scale, can give rise to mixings of the order $\epsilon \sim 10^{-13}$. Tantalizingly, this tiny mixing is compatible with the asymmetric reheating mechanism while providing an achievable sensitivity goal for direct detection experiments.

In this paper we explore the possibility of discovering the twin baryons and electrons of the MTH scenario in current and next-generation direct detection experiments. We consider a framework in which mirror matter is sub-nano-charged, as a consequence of kinetic mixing between the hypercharge gauge boson of the SM and its massless mirror counterpart. For concreteness, we assume that the discrete $\mathbb{Z}_{2}$ twin symmetry is only softly broken, so that the masses of mirror particles are fixed by the ratio of electroweak VEVs in the two sectors, $\hat{v} / v$. The constraint on this scenario from $\Delta N_{\text {eff }}$ is assumed to be satisfied as a consequence of late-time asymmetric reheating. Since mirror matter, like visible matter, is dissipative, some fraction of the twin DM in the galaxy may have collapsed into a disk. The direct detection signal then depends in part on whether a twin disk is present and, if so, the fraction of mirror matter it contains, its alignment relative to the visible disk, and whether it extends out to the location of the Earth. The size of the signal also depends on whether the mirror matter in the galaxy is in ionic form or has condensed into atoms.

In order to understand the distribution of mirror matter in the galaxy, and whether it is in the form of ions or atoms, it is necessary to track how this subcomponent of DM evolved in time as the Milky Way was forming. When halo formation begins at redshifts of $\mathcal{O}(10)$, the shock wave induced by the in-falling twin atoms heats up and reionizes the mirror sector. The mirror sector can dissipate its energy through the emission of twin photons in processes involving the scattering of twin particles. The timescale of this cooling process depends on the abundance of mirror particles and is longer than in the SM. We find that this timescale can nevertheless be shorter than the age of the universe for sufficiently large abundances of mirror matter. This indicates that some fraction of the mirror halo may have collapsed into a disk.

In our analysis we consider experiments based on both nuclear recoil (NR) and electron recoil (ER) signals. For a given mirror DM abundance we determine the expected event rates in direct detection experiments for the limiting cases of an ionized halo, an ionized disk, an atomic halo and an atomic disk. We are careful to account for the effects of mirror matter capture in the Earth. We find that in most of the relevant parameter space, its effect on the direct detection signals we consider is negligible or at most modest. By taking advantage of the complementarity of the different experiments, we find that it may be possible to establish not just the multi-component nature of mirror DM, but also its distribution in the galaxy. In addition, a study of the recoil energies may be able to determine the masses of the mirror DM constituents. By establishing that the masses and charges of mirror hydrogen and helium are integer multiples of each other, these experiments may be able to diagnose the mirror nature of the theory. There is also an important complementarity between direct detection experiments and astrophysical probes of mirror matter. The reach 
of the former is best for mirror baryons arranged in a halo. On the other hand, dark disk scenarios can be probed very sensitively via white dwarf cooling bounds [65], and are also more likely to lead to the formation of mirror stars, which can be detected in optical and $X$-ray observations [66, 67], microlensing surveys [68], and gravitational wave observations of mirror neutron star mergers [69].

Although our focus is on MTH models with softly broken twin symmetry, the direct detection signatures we study are also features of the more general class of models in which mirror baryons and electrons constitute some or all of the observed DM. Earlier work on the distribution of mirror DM in the galaxy may be found, for example, in [70-72]. Direct detection of mirror matter has been considered, for example, in [73-77]. Reviews of mirror models and mirror DM, with many additional references, may be found in [78-81]. In detail, however, the direct detection signals depend sensitively on the masses of the mirror particles and their distribution in the galaxy. From this perspective, our paper represents a detailed study of the direct detection signals of generalized mirror-like models in the region of parameter space motivated by the little hierarchy problem. From an even more general perspective, mirror DM models in all their incarnations fall into the broader class of atomic DM models [82-95]. Since the motivations for the existence of a small dark photon kinetic mixing portal apply just as well to any atomic DM model, these theories could therefore give rise to similar signals in direct detection experiments. Almost all aspects of our analysis can be carried over to the study of the direct detection signals of this more general class of models. This includes our framework for determining the distribution of this form of DM in our galaxy and the consequent implications for the different types of direct detection experiments, and our study of the potentially large effects arising from capture of DM in the Earth. Our analysis lays the groundwork for future studies that will greatly enhance our understanding of general theories of atomic dark matter.

The outline of this paper is as follows. In the next section we give a quick review of the parameter space of the MTH model. In section 3 we study the distribution of mirror particles in the Milky Way, based on an estimate of the rate of twin particle cooling after the shock wave heating process. In section 4 , we estimate the signal rates in direct detection experiments, considering both nuclear and electron recoils. Our conclusions are in section 5 . Mirror matter capture in the Earth and its effects on direct detection are carefully analyzed in appendix A.

\section{Parameters of the model}

Our focus is on the direct detection signals of MTH models in which the mirror nuclei and electrons constitute a subcomponent of DM. We restrict our analysis to the case when the Yukawa couplings respect the discrete $Z_{2}$ symmetry that relates the two sectors. Then the elementary fermions in the twin sector are heavier than their visible counterparts by a factor of $\hat{v} / v$, the ratio of electroweak symmetry breaking scales in the two sectors. The energy density in twin radiation is assumed to be diluted by late time asymmetric reheating after the two sectors have decoupled, allowing the current CMB and BBN constraints on 
dark radiation to be satisfied. ${ }^{1}$ Then, in this framework, the direct detection signals depend on four parameters,

$$
\epsilon, \quad \hat{v} / v, \quad r_{\text {all }}=\Omega_{\text {all mirror baryons }} / \Omega_{\mathrm{DM}}, \quad \hat{Y}_{p}\left({ }^{4} \hat{\mathrm{He}}\right)=\frac{\rho_{4} \hat{\mathrm{He}}}{\rho_{\hat{\mathrm{H}}}+\rho_{4} \hat{\mathrm{He}}} .
$$

Here $\epsilon$ parametrizes the kinetic mixing between the hypercharge gauge bosons in the two sectors, while $r_{\text {all }}$ denotes the total asymmetric mirror baryon density relative to the total DM density today. Just as in the case of the SM, the contributions of the twin sector to the matter density are almost entirely from mirror hydrogen and helium,

$$
r_{\text {all }}=r_{\hat{\mathrm{H}}}+r_{\hat{\mathrm{He}}} \cdot
$$

The parameter $\hat{Y}_{p}\left({ }^{4} \hat{\mathrm{He}}\right)$ represents the mass fraction contributed by twin helium.

The masses of particles in the twin sector depend on the ratio $\hat{v} / v$. While Higgs coupling measurements at the LHC constrain $\hat{v} / v \gtrsim 3$, the requirement that the Higgs mass be only modestly tuned limits $\hat{v} / v \lesssim 5$. The mass of the twin electron is simply $\hat{v} / v$ times the corresponding value in the SM. Since the quark masses are also $\hat{v} / v$ times larger than in the $\mathrm{SM}$, the different running of the mirror QCD gauge coupling leads to a larger confinement scale in the mirror sector than in the SM by about $30-50 \%$ in the range $\hat{v} / v=3-5$. This makes twin baryons heavier than SM baryons by about 30-50\% [62].

Since mirror particles constitute an acoustic subcomponent of DM, they lead to a suppression of large scale structure on scales that enter the horizon prior to recombination in the twin sector. This can be used to place limits on the contribution of mirror matter to the observed density of DM, $r_{\text {all }} \lesssim 10 \%$ [62].

The relative fractions of mirror hydrogen and helium in the early universe are determined by the dynamics of BBN in the twin sector. This in turn depends on the masses of the mirror baryons and also on the energy density in mirror radiation at the time of BBN. In [62], the Boltzmann equations for the number changing process $\hat{n} \hat{\nu} \leftrightarrow \hat{p} \hat{e}$ were solved for the MTH model, and the timescale for mirror deuterium formation was determined. It was found that $\hat{Y}_{p}\left({ }^{4} \hat{\mathrm{He}}\right) \approx 75 \%$ for $\hat{v} / v$ in the range we consider and realistic values of $\Delta N_{\text {eff }}$. However, in our analysis we also consider the cases in which the twin baryons are composed entirely of mirror hydrogen or mirror helium, corresponding to $\hat{Y}_{p}\left({ }^{4} \hat{\mathrm{He}}\right)=0$ and $\hat{Y}_{p}\left({ }^{4} \hat{\mathrm{He}}\right)=1$. These provide some insight into the direct detection signals of MTH models in which the Yukawa couplings of the light quarks exhibit hard breaking of the discrete $Z_{2}$ symmetry, so that the spectrum of mirror nuclei is composed of only a single species, either hydrogen or helium. This allows us to explore the interesting scenario in which the mirror neutron is lighter than the mirror proton and constitutes the primary component of DM [2], while any remaining mirror protons are contained in a subcomponent of DM composed entirely of twin helium. In this framework, the signals we discuss arise entirely from the mirror helium subcomponent. This scenario is captured in our $\hat{Y}_{p}\left({ }^{4} \hat{\mathrm{He}}\right)=1$ analysis with the understanding that $r_{\text {all }}$ is to be interpreted, not as the fractional contribution of mirror baryons to the DM density, but as the fractional contribution of twin helium alone. Mirror baryons could therefore account for all of the dark matter in our universe today, opening an avenue to explaining the comparable relic densities of DM and baryons [96, 97].

\footnotetext{
${ }^{1}$ We assume the masses of both visible and hidden sector neutrinos can be neglected.
} 


\section{Mirror baryon distribution in the Milky Way}

Structure formation reaches the regime of nonlinear halo formation at redshifts $z \sim \mathcal{O}(10)$ [98]. At these redshifts, DM and the SM particles, which include nuclei, electrons, and photons, undergo complicated collective dynamics that gives rise to the structure of the Milky Way and the other galaxies that we observe today. Collisionless DM particles clump under the action of gravity, eventually giving rise to cold DM (CDM) distributions such as the NFW or Burkert profiles (see e.g. [99]). The SM baryons, which are initially bound in atoms following recombination, fall into the overdense regions and collide with each other, leading to the formation of a shock wave that expands outwards to heat the baryonic medium. The maximum temperature of the baryons is dictated by the virial theorem and the available gravitational energy, which is dominated by the CDM halo. The immediate aftermath of shock heating is a fully ionized baryon distribution that is in hydrostatic equilibrium. This distribution initially satisfies the adiabatic equation of state, but quickly evolves to reach thermal equilibrium. Subsequently, processes such as bremsstrahlung and ionization cooling lower the temperature of the baryons, leading to a loss of pressure support and the eventual onset of catastrophic collapse. If the halo has sufficient angular momentum and a quiet merger history, this collapse eventually gives rise to a disk such as the one in our own Milky Way galaxy. Even after the disk has formed, a significant fraction of the baryonic gas remains outside the disk at large distances from the galactic core [100].

If mirror baryons make up a small $\lesssim \mathcal{O}(10 \%)$ fraction of DM, we expect that, during halo formation, they will undergo broadly similar dynamics to SM baryons. Mirror atoms will also fall into overdense regions, undergo shock heating and ionization, and reach adiabatic equilibrium before eventually settling into thermal equilibrium. They then cool, potentially leading to collapse and the formation of a mirror disk. This kind of dynamics for a DM component has been studied in the past, primarily in the context of exact mirror DM models that are perfect hidden sector copies of the SM, though there have been some early studies of how cosmology and galactic evolution might have proceeded for mirror matter with a larger mirror Higgs vev than in the SM [70]. More recently, this scenario has also been explored in the more general context of dissipative DM models that could form a so-called 'dark disk' [87, 88, 101, 102], though without the presence of 'dark nuclear physics' which, as we describe below, can significantly complicate galactic evolution in mirror models.

In spite of this general understanding, it is not possible to make precise predictions about the distribution of mirror DM in the galaxy in the MTH scenario. Even in the case of ordinary baryonic matter, collapse and disk formation is a highly nonlinear process that depends sensitively on various radiative and mechanical feedback mechanisms including star formation, stellar winds, and heating from supernovae, as well as the influence of the central supermassive black hole. Even for visible matter, modelling these processes requires detailed $N$-body simulations incorporating magnetohydrodynamics and stellar feedback [103-114]. While the formation of our Milky Way disk is beginning to be better understood, the simulations are not yet fine-grained enough to make direct contact with the astrophysics of individual stars, let alone fundamental physics parameters. Instead, 
these simulations rely on large-scale parameterizations of processes like star formation and heating from supernovae explosions to reproduce the known Milky Way structure. Given that the particle spectrum and dynamics of the MTH are different enough from the SM that detailed analogies break down, it is not feasible to robustly predict the distribution of mirror baryons in the galaxy.

We therefore take a more modest approach. In this section, we compute the mirror baryon distributions resulting from hydrostatic equilibrium in the gravitational background of the CDM halo (neglecting the effects of halo angular momentum). These distributions can be interpreted as the rough starting point for the nonlinear processes of collapse. By examining the cooling rates arising from various processes and comparing to the predictions for SM-like baryons computed under the same assumptions, we can obtain some insight into how the mirror baryon distribution might be expected to evolve in our Milky Way. The most important question is whether the mirror matter collapses to form its own disk. We find that in a large part of the parameter range, because of the large uncertainties, the answer is ambiguous. For this reason, in our study of direct detection in section 4, we consider both halo and disk distributions of mirror matter.

\subsection{Initial mirror baryon distribution}

In this subsection we compute the initial mirror matter distribution in our Milky Way prior to the onset of cooling, assuming a standard NFW or Burkert CDM distribution for the primary DM component and hydrostatic equilibrium. This will allow us to estimate the cooling timescale in section 3.2. We pay particular attention to how the distribution of mirror baryons compares to that of SM baryons computed under the same assumptions. This will give us some insight into how the cooling timescale of mirror baryons, and consequently their current distribution, might be expected to differ from that of the SM baryons in our Milky Way.

\subsubsection{CDM profile}

We assume that the mirror particles contribute only a small component of the total energy density in DM, which is dominated by standard CDM with distribution $\rho_{\mathrm{CDM}}(r)$. Then the contribution of mirror particles to the gravitational potential can be neglected. To examine the sensitivity of our results on the CDM distribution, we consider two possibilities, an NFW profile and a Burkert profile,

$$
\begin{aligned}
\rho_{\mathrm{NFW}}(r) & =\frac{\rho_{H}}{\frac{r}{R_{H}}\left(1+\frac{r}{R_{H}}\right)^{2}}, \\
\rho_{\mathrm{BUR}}(r) & =\frac{\rho_{H}}{\left(1+\frac{r}{R_{H}}\right)\left(1+\frac{r^{2}}{R_{H}^{2}}\right)} .
\end{aligned}
$$

The benchmark parameters we assume are based on the studies in [99], and are summarized in table 1. To facilitate comparison, we assume common values for $R_{\odot}$, the distance of the Sun from the Milky Way center, and the local CDM density $\rho_{\odot}=\rho\left(R_{\odot}\right)$. The profiles are 


\begin{tabular}{|l|l|l|l|l|l|}
\hline Profile & $R_{\odot}$ & $\rho(\odot)$ & $R_{H}$ & $R_{\text {vir }}$ & $T_{\text {vir }} / \bar{m}_{\text {halo }}$ \\
\hline NFW & 8 & 0.5 & 16 & 235 & $1.35 \times 10^{-7}$ \\
\hline Burkert & 8 & 0.5 & 9 & 209 & $1.21 \times 10^{-7}$ \\
\hline
\end{tabular}

Table 1. Parameters of CDM distributions, which dominate the gravitational potential of the Milky Way. All distances in $\mathrm{kpc}, \rho$ in $\mathrm{GeV} / \mathrm{cm}^{3} \cdot \bar{m}_{\text {halo }}$ is the halo-averaged mass of a virialized sub-population of the halo, such as the MTH mirror baryons.

then completely fixed by specifying the remaining parameter $R_{H} \cdot{ }^{2}$ For $R>R_{\odot}$, the NFW and Burkert profiles are very similar, but the NFW profile predicts much higher densities closer to the core. These profiles are shown as black curves in figure 1 (top).

Let $M_{\mathrm{CDM}}(R)$ represent the total mass of DM enclosed within radius $R$. We define the total size of the halo by the virial radius $R_{\mathrm{vir}}$. This is determined from the standard overdensity criterion, $R_{\mathrm{vir}} \equiv R_{200}$, as

$$
M_{\mathrm{CDM}}\left(R_{200}\right)=\Delta \cdot \rho_{\text {crit }} \cdot \frac{4 \pi}{3} R_{200}^{3},
$$

where $\Delta=200$ and $\rho_{\text {crit }} \approx 4.8 \times 10^{-6} \mathrm{GeV} / \mathrm{cm}^{3}$ is the critical density.

The virial theorem allows us to relate the average kinetic and potential energy of CDM particles, $\frac{1}{2}\left|U_{\text {tot }}\right|=\mathrm{KE}_{\text {tot }}=\frac{1}{2} M\left(R_{\text {vir }}\right) v_{0}^{2}$. We can then determine the average velocity-squared of the particles that constitute the primary component of DM,

$$
v_{0}^{2}=\frac{\int_{0}^{R_{\mathrm{vir}}} G M_{\mathrm{CDM}}(r) \rho_{\mathrm{CDM}}(r) r d r}{\int_{0}^{R_{\mathrm{vir}}} \rho_{\mathrm{CDM}}(r) r^{2} d r} .
$$

If mirror matter were collisionless, the mirror atoms would exhibit the same distribution as the primary DM component, with the same root-mean-square velocity. On timescales short compared to the cooling timescale, the only effect of collisions is to redistribute the energy of the mirror particles amongst themselves, leaving their total energy unchanged. In what follows, we use this fact, together with the condition of hydrostatic balance, to determine the distribution of mirror matter in adiabatic and thermal equilibrium prior to the onset of cooling.

\subsubsection{Mirror matter in hydrostatic equilibrium}

The mirror baryons, like the SM baryons, are shock heated and ionized as they fall into the collapsing CDM halo. During this process, and immediately afterwards, the mirror baryons remain well mixed. Matter is churned around in the profile at the convection timescale,

$$
t_{\text {convection }}(r) \sim \sqrt{\frac{1}{G \rho_{\mathrm{CDM}}(r)}} \sim 10^{8} \mathrm{yrs} \quad \text { at } r=R_{\odot} .
$$

See also figures 2 and 3. This is comparable to the time scale when the non-linear halo formation sets in at $z=\mathcal{O}(10)$, so convection quickly establishes the mirror baryons in an

\footnotetext{
${ }^{2}$ We have explored the effect of the uncertainties on the fitted parameters of a given CDM profile on our results and found them to be negligible compared to the difference between these two profiles.
} 
adiabatic distribution. In this configuration, the mirror matter forms a dark plasma that is pressure supported while it remains hot enough to stay ionized. However, as we shall see, the distribution quickly evolves to become isothermal, on a timescale dictated by the diffusion timescale in the adiabatic profile.

In computing the mirror halo profiles, we will assume that mirror hydrogen and helium are fully ionized. The fraction of partially or fully recombined mirror baryons is far too small to affect the calculation of mirror density and temperature profiles. It does however have an important impact on the various cooling mechanisms considered in section 3.2.1. We will therefore self-consistently determine the actual degree of ionization consistent with the computed mirror baryon profiles in section 3.1.4. We define the average mass of the mirror matter particles at any location in the halo as

$$
\bar{m}(\vec{r}) \equiv \frac{\sum_{i} n_{i}(\vec{r}) m_{i}}{\sum_{i} n_{i}(\vec{r})}
$$

where $i$ runs over mirror hydrogen and helium, and also over the electrons. Approximating $m_{\hat{\mathrm{He}}}=4 m_{\hat{\mathrm{H}}}$, the resulting local average mass of mirror particles in the limit of full ionization is given by,

$$
\bar{m}(\vec{r})=m_{\hat{\mathrm{H}}} \frac{4}{8-5 \hat{Y}(\vec{r})},
$$

where $\hat{Y}(\vec{r})$ is the local mirror helium mass fraction.

Initially, the mirror baryons and electrons are well-mixed, so that $\bar{m}$ has the same value $\bar{m}_{\text {halo }}$ everywhere in the halo. The value of $\bar{m}_{\text {halo }}$, the average mirror particle mass over the whole halo, can be obtained from eq. (3.7) by assuming that the total mirror helium mass fraction follows the cosmological average $\hat{Y}_{p}\left({ }^{4} \hat{\mathrm{He}}\right)$,

$$
\bar{m}_{\text {halo }}=m_{\hat{\mathrm{H}}} \frac{4}{8-5 \hat{Y}_{p}\left({ }^{4} \hat{\mathrm{H}} \mathrm{e}\right)} .
$$

We now determine the distribution of mirror particles in the adiabatic configuration. We start from the observation that in both the adiabatic and isothermal configurations the mirror baryons satisfy the condition of hydrostatic equilibrium in the gravitational background of the CDM distribution,

$$
\frac{d P}{d r}=-\frac{G M_{\mathrm{CDM}}(r) \rho(r)}{r^{2}} .
$$

Here $P(r)$ and $\rho(r)$ are the local pressure and density of the mirror particles, which are related by their equation of state. The density $\rho(r)$ can be determined from the local number densities of the mirror particles.

The next step is to determine the average energy of a mirror particle prior to cooling. Initially, mirror DM and the primary component of DM are well-mixed. Since the primary component of DM dominates the gravitational potential of the halo, a small density of mirror matter would arrange itself in exactly the same distribution as the primary component if the mirror particles were collisionless. In this scenario, the average energy of a mirror particle would be $-(3 / 2) T_{\text {vir }}$, with $T_{\text {vir }} / \bar{m}_{\text {halo }} \sim 10^{-7}$, see table 1 . The dominant effect of collisions 
between the mirror particles is just to redistribute their energy amongst themselves, so that their total energy is conserved, up to the small fraction of energy that is used to ionize the mirror atoms. The cooling timescale is long compared to the timescales for convection or diffusion. This means that for mirror particles in an adiabatic or isothermal halo, the average energy per particle (the sum of kinetic and potential energy) is still given by $-(3 / 2) T_{\text {vir }}$ minus the average ionization energy. We now use this fact, together with the condition of hydrostatic equilibrium, eq. (3.9), to determine the distribution of mirror particles in an adiabatic halo prior to cooling.

Initially the mirror baryons are uniformly mixed, so that $\hat{Y}(r)$ is equal to the cosmic value $\hat{Y}_{p}\left({ }^{4} \hat{\mathrm{He}}\right)$ and $\bar{m}$ constant throughout and equal to $\bar{m}_{\text {halo }}$. In the adiabatic regime, the mirror baryons obey the equation of state $P=A \rho^{\gamma}$ with $\gamma=5 / 3$ for a monoatomic gas, where $A$ is a constant independent of position. The total number density $n(r)$ is related to the local density $\rho(r)$ as $n(r)=\rho(r) / \bar{m}_{\text {halo }}$. The ideal gas law $\bar{m}_{\text {halo }} P(r)=\rho(r) T(r)$ then yields,

$$
\rho(r)=\left(\frac{A \bar{m}_{\text {halo }}}{T(r)}\right)^{\frac{1}{1-\gamma}},
$$

which relates the density profile to the temperature. Hydrostatic equilibrium then allows us to relate the temperature at an arbitrary point in the halo to the temperature at the center,

$$
T(r)=T(0)-G \bar{m}_{\text {halo }}\left(\frac{\gamma-1}{\gamma}\right) \int_{0}^{r} \frac{M_{\mathrm{CDM}}(\tilde{r})}{\tilde{r}^{2}} d \tilde{r} .
$$

The temperature at the center of the distribution $T(0)$ is obtained by requiring that the average total energy of a mirror particle in the halo is $-(3 / 2) T_{\text {vir }}$, up to small corrections arising from the ionization energies of the atoms.

The adiabatic distribution quickly becomes isothermal, on timescales dictated by the diffusion process in the adiabatic profile. We can estimate the diffusion timescale by focusing on scattering between ionized $\hat{X}=\hat{\mathrm{H}}^{+}, \hat{\mathrm{He}}^{+,++}$, which can transfer heat more efficiently between ions than $\hat{X} \hat{e}$ scattering. The scattering cross section between $\hat{X}$ 's can be estimated as $\sigma \sim \alpha^{2} / T^{2}$. For diffusion with a free streaming length $\lambda_{F S} \sim\left(\rho_{\text {mirror }} \sigma / \bar{m}_{\text {halo }}\right)^{-1}$, the number of scatterings involved in moving in a random walk across a distance $L$ can be estimated as $N \sim\left(L / \lambda_{F S}\right)^{2}$. Since the time it takes to undergo $N$ scatterings is of order $t \sim N \lambda_{\mathrm{FS}} / v_{\hat{X}}$, the characteristic timescale for diffusing through a distance $L$ is given by,

$$
t_{\text {diffusion }}(r, L) \sim \frac{\rho_{\text {mirror }} \sigma}{\bar{m}_{\text {halo }} v_{\hat{X}}} L^{2} .
$$

The diffusion timescale in the adiabatic profile depends only modestly on $r$ and is in the range $\sim 10^{6}-10^{8}$ years for $L \sim 10 \mathrm{kpc}$. It follows that the adiabatic gas quickly reaches thermal equilibrium and arranges itself in an isothermal distribution. We will therefore use the isothermal mirror halo distributions in our discussion of cooling in the next section.

We now determine the distribution of mirror matter in an isothermal halo prior to the onset of cooling. The isothermal halo is at a constant temperature $T=T_{\text {iso }}$, but $\hat{Y}(r)$ and $\bar{m}(r)$ are now spatially dependent since constituents with different atomic weights settle at different distances from the center. 
For an isothermal distribution, the hydrostatic equilibrium condition eq. (3.9) applies separately for different gas components $X$. Charge separation due to the different masses of mirror nuclei and electrons occurs on scales of the Debye length, which is negligible on galactic scales. It is therefore an excellent approximation to define the two dominant components of the mirror baryon distribution to be $\hat{X}=\left\{\hat{\mathrm{H}}^{+}+\hat{\mathrm{e}}^{-}, \hat{\mathrm{H}} \mathrm{e}^{++}+2 \hat{e}^{-}\right\}$, which allows us to only consider gravitational forces when solving for hydrostatic equilibrium. Applying the ideal gas law for each of these two components gives a partial pressure $P_{X}$ that is $\left(1+Q_{\hat{X}}\right)$ times higher than the pressure for neutral mirror hydrogen or helium atoms, where $Q_{\hat{X}}=1$ (2) for $\hat{\mathrm{H}}^{+}\left(\hat{\mathrm{H}} \mathrm{e}^{++}\right)$[115]. The solution to eq. (3.9) for each component is therefore

$$
n_{\hat{X}}(r)=n_{\hat{X}}(0) \exp \left[-\frac{G m_{\hat{X}}}{T_{\text {iso }}} \frac{1}{1+Q_{\hat{X}}} \int_{0}^{r} \frac{M_{\mathrm{CDM}}(\tilde{r})}{\tilde{r}^{2}} d \tilde{r}\right] .
$$

The number densities $n_{X}(0)$ at the center of the halo and the temperature $T_{\text {iso }}$ are determined from $r_{\text {all }}$ and $\hat{Y}$, and the condition that the average energy of mirror particles in the halo is $-(3 / 2) T_{\text {vir }}$ minus the energy used to ionize the mirror atoms. The isothermal distribution has a greater density of matter near the center of the halo than the corresponding CDM distribution. The corresponding reduction in the gravitational potential energy results in an increase in temperature, so that $T_{\text {iso }}$ is a factor of about 2 larger than the virial temperature $T_{\text {vir }}$.

\subsubsection{Results}

As we have seen, the gas of mirror particles quickly evolves to reach thermal equilibrium, and arranges itself in an isothermal distribution. We will therefore use the isothermal mirror halo distributions as the basis for our discussion of cooling. In general, the form of the isothermal distribution depends on $r_{\text {all }}, \hat{v} / v$ and $\hat{Y}_{p}\left({ }^{4} \hat{\mathrm{He}}\right)$. However, the dependence of the mirror baryon profile on $r_{\text {all }}$ is simple; the density scales proportionally with $r_{\text {all }}$ as long as the mirror sector contribution to the gravitational potential can be neglected. Similarly, the effect of $\hat{v} / v$ on the distribution is very minor for values in the range of interest. However, the dependence on the mirror helium fraction $\hat{Y}_{p}\left({ }^{4} \hat{\mathrm{He}}\right)$ is non-trivial, and we therefore focus on illustrating this. To this end, we show the profiles for three different MTH benchmark points. For each benchmark point the values of $\hat{v} / v=3$ and $r_{\text {all }}=0.01$ are held fixed, but we consider three different values of the helium fraction, $\hat{Y}_{p}\left({ }^{4} \hat{\mathrm{He}}\right)=0.75$ (close to the BBN prediction for the asymmetrically reheated MTH scenario [62]), and the limiting cases of $\hat{Y}_{p}\left({ }^{4} \hat{\mathrm{He}}\right)=0$ and $\hat{Y}_{p}\left({ }^{4} \hat{\mathrm{He}}\right)=1$ (pure mirror hydrogen and helium). To serve as a basis for comparison, we also compute a profile representative of SM baryons, corresponding to the MTH parameters $\hat{Y}_{p}\left({ }^{4} \hat{\mathrm{He}}\right)=0.25, r_{\text {all }}=0.2$ and $\hat{v} / v=1$.

Table 2 summarizes the isothermal mirror baryon halo parameters at the location of our Sun, while spatial distributions are shown in figure 1 . We define

$$
\begin{aligned}
& \hat{Y}_{\odot}=\hat{Y}\left(R_{\odot}\right) \\
& v_{\odot}=v_{0}\left(R_{\odot}\right)
\end{aligned}
$$

to be the local values of the mirror helium fraction and mirror baryon velocity dispersion respectively. Temperature and ionization are constant throughout the halo, and both 


\begin{tabular}{|c|c|c|c|c|c|c|c|}
\hline Scenario (NFW) & $\frac{T_{\text {iso }}}{\mathrm{eV}}$ & $\frac{v_{\odot}}{\mathrm{km} / \mathrm{s}}$ & $\left(\chi_{\mathrm{H}^{+}}, \chi_{\mathrm{He}^{+}}, \chi_{\mathrm{He}^{++}}\right)$ & $\frac{\rho_{\mathrm{CDM}}}{\mathrm{GeV} / \mathrm{cm}^{3}}$ & $\frac{\rho_{\text {mirror }}}{\mathrm{GeV} / \mathrm{cm}^{3}}$ & $\frac{1}{r_{\text {all }}} \frac{\rho_{\text {mirror }}}{\rho_{\mathrm{CDM}}}$ & $\hat{Y}_{\odot}$ \\
\hline $\begin{array}{l}\text { SM Baryons } \\
r_{\text {all }}=0.01\end{array}$ & 173 & 197 & $\left(1,1.7 \times 10^{-7}, 1\right)$ & 0.5 & 0.12 & 1.29 & 0.96 \\
\hline $\begin{array}{l}\frac{\hat{v}}{v}=3, r_{\text {all }}=0.01 \\
\hat{Y}_{p}\left({ }^{4} \hat{\mathrm{He}}\right)=0.75\end{array}$ & 309 & 230 & $\left(1,4 \times 10^{-7}, 1\right)$ & 0.5 & 0.004 & 0.77 & 0.992 \\
\hline $\begin{array}{l}\frac{\hat{v}}{v}=3, r_{\text {all }}=0.01 \\
\hat{Y}_{p}\left({ }^{4} \hat{\mathrm{He}}\right)=0\end{array}$ & 142 & 253 & $(1,-,-)$ & 0.5 & 0.0019 & 0.39 & 0 \\
\hline $\begin{array}{l}\frac{\hat{v}}{v}=3, r_{\text {all }}=0.01 \\
\hat{Y}_{p}\left({ }^{4} \hat{\mathrm{He}}\right)=1\end{array}$ & 369 & 249 & $\left(-, 3 \times 10^{-7}, 1\right)$ & 0.5 & 0.0021 & 0.42 & 1 \\
\hline
\end{tabular}

\begin{tabular}{|c|c|c|c|c|c|c|c|}
\hline Scenario (Burkert) & $\frac{T_{\text {iso }}}{\mathrm{eV}}$ & $\frac{v_{\odot}}{\mathrm{km} / \mathrm{s}}$ & $\left(\chi_{\mathrm{H}^{+}}, \chi_{\mathrm{He}^{+}}, \chi_{\mathrm{He}^{++}}\right)$ & $\frac{\rho_{\mathrm{CDM}}}{\mathrm{GeV} / \mathrm{cm}^{3}}$ & $\frac{\rho_{\text {mirror }}}{\mathrm{GeV} / \mathrm{cm}^{3}}$ & $\frac{1}{r_{\text {all }}} \frac{\rho_{\text {mirror }}}{\rho_{\mathrm{CDM}}}$ & $\hat{Y}_{\odot}$ \\
\hline $\begin{array}{l}\text { SM Baryons } \\
r_{\text {all }}=0.01\end{array}$ & 145 & 181 & $\left(1,2 \times 10^{-7}, 1\right)$ & 0.5 & 0.12 & 1.22 & 0.95 \\
\hline $\begin{array}{l}\frac{\hat{v}}{v}=3, r_{\text {all }}=0.01 \\
\hat{Y}_{p}\left({ }^{4} \hat{\mathrm{He}}\right)=0.75\end{array}$ & 263 & 212 & $\left(1,5 \times 10^{-7}, 1\right)$ & 0.5 & 0.0039 & 0.78 & 0.99 \\
\hline $\begin{array}{l}\frac{\hat{v}}{v}=3, r_{\text {all }}=0.01 \\
\hat{Y}_{p}\left({ }^{4} \hat{\mathrm{He}}\right)=0\end{array}$ & 119 & 231 & $(1,-,-)$ & 0.5 & 0.0021 & 0.42 & 0 \\
\hline $\begin{array}{l}\frac{\hat{v}}{v}=3, r_{\text {all }}=0.01 \\
\hat{Y}_{p}\left({ }^{4} \hat{\mathrm{He}}\right)=1\end{array}$ & 311 & 229 & $\left(-, 4 \times 10^{-7}, 1\right)$ & 0.5 & 0.0023 & 0.46 & 1 \\
\hline
\end{tabular}

Table 2. Isothermal halo parameters at $r=R_{\odot}=8 \mathrm{kpc}$ for some benchmark mirror baryon scenarios. $v_{\odot}$ is the mean velocity of mirror baryon constituents following a thermal distribution of temperature $T_{\text {iso }}$. Temperature and ionization is constant throughout the halo. For each $\hat{Y}_{p}\left({ }^{4} \mathrm{He}\right)$ separately, the local mirror helium fraction $\hat{Y}_{\odot}$, local mean velocity $v_{\odot}$, and the local $\frac{1}{r_{\text {all }}} \frac{\rho_{\text {mirror }}}{\rho_{\mathrm{CDM}}}$ are almost independent of $\hat{v} / v$ and $r_{\text {all }}$, see text for discussion. As the halo cools, $\hat{Y}_{\odot}$ will be reduced as mirror helium sinks to the bottom of the gravity well. The SM comparison scenario corresponds to evaluating the mirror baryon profile for $\hat{v} / v=1, r_{\text {all }}=0.2, \hat{Y}_{p}\left({ }^{4} \hat{\mathrm{He}}\right)=0.25$. This is presented to compare mirror halo parameters to a SM-like halo evaluated under the same assumptions, but does not represent the actual SM baryon distribution today. 

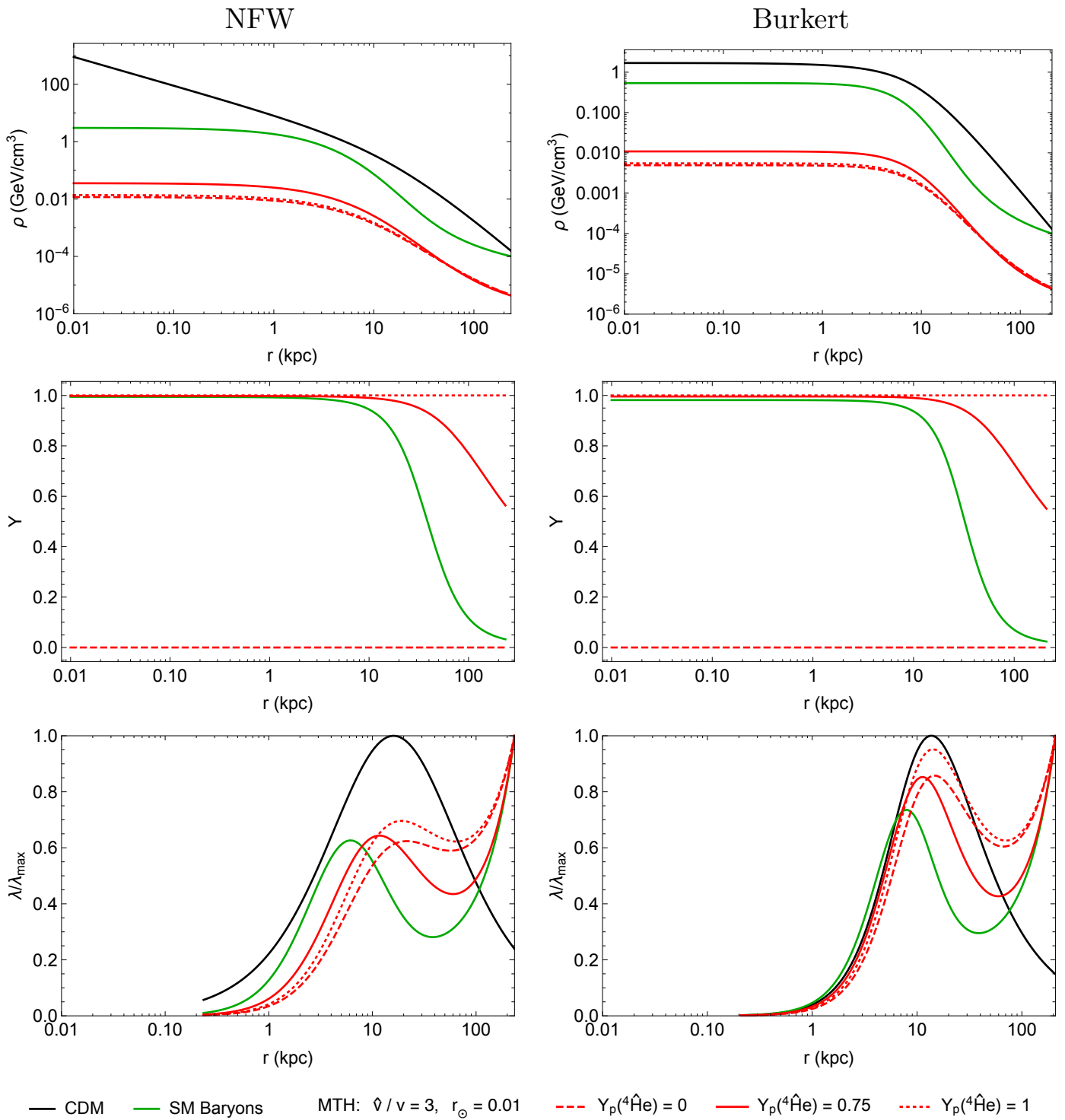

Figure 1. Top: density profile for the mirror halo benchmarks in table 2, evaluated in the background of the NFW or Burkert CDM Profile. Middle: mirror helium mass fraction $\hat{Y}(r)$ as a function of $r$. Bottom: mass density per spherical shell $\lambda=4 \pi r^{2} \rho(r)$, normalized to its maximum value for each profile. This shows the distance where most of the constituent atoms are situated. The SM comparison scenario corresponds to evaluating the mirror baryon profile for $\hat{v} / v=1, r_{\text {all }}=0.2, Y=0.25$. This is presented to compare mirror halo parameters to a SM-like halo evaluated under the same assumptions, but does not represent the actual SM baryon distribution today. 
mirror hydrogen and helium are almost completely ionized. The local mean velocity $v_{\odot}$ is comparable to, although somewhat higher than, the canonical CDM halo expectation of $\sim 220 \mathrm{~km} / \mathrm{s}$. The local density of mirror baryons differs from $r_{\text {all }} \cdot \rho_{\mathrm{CDM}}$ by an $\mathcal{O}(1)$ factor. For $\hat{Y}_{p}\left({ }^{4} \hat{\mathrm{He}}\right) \neq 0,1$, the local mirror helium fraction $\hat{Y}_{\odot}$ is highly temperature dependent. A reduction in the halo temperature by a factor of two could reduce $\hat{Y}_{\odot}$ to almost zero as the region of helium-dominance, see figure 1 (middle), retreats towards the center of the gravity well. Therefore, any variation in the halo results in a large change in the local value of $\hat{Y}_{\odot}$.

The four chosen benchmarks are representative of the behavior in the MTH framework. For each $\hat{Y}_{p}\left({ }^{4} \hat{\mathrm{He}}\right)$ separately, the local values of $\hat{Y}_{\odot}, v_{\odot}$ and $\left(1 / r_{\text {all }}\right)\left(\rho_{\text {mirror }} / \rho_{\mathrm{CDM}}\right)$, as well as the halo temperature $T_{\text {iso }}$, are relatively insensitive to the values of $\hat{v} / v$ and $r_{\text {all }} .{ }^{3}$ On the other hand, the dependence of the profiles on $\hat{Y}_{p}\left({ }^{4} \hat{\mathrm{He}}\right)$ is nontrivial. In particular, the SM-like value, $\hat{Y}_{p}\left({ }^{4} \hat{\mathrm{He}}\right)=0.25$, is close to optimal for concentrating mirror baryons near the galactic center, with larger or smaller values leading to puffier profiles. As we shall see, this interesting coincidence has important implications for the cooling rates.

\subsubsection{Mirror baryon ionization fractions}

Before the onset of cooling, the mirror halo is sufficently hot that the mirror baryons are very close to fully ionized. ${ }^{4}$ However, the small fraction of partially or fully recombined mirror helium and hydrogen is important for non-bremsstrahlung cooling processes. We therefore now discuss how to determine the degree of ionization for the computed mirror baryon profiles.

In general, we wish to determine the local ionization fractions assuming some local temperature and number densities of mirror hydrogen and helium. If the conditions of detailed balance are satisfied, as in the early universe, these ionization fractions can be obtained from Saha's equation [98]. However, mirror halos are usually optically thin, meaning that photons emitted from bremsstrahlung or atomic cooling processes escape the galaxy. In such a scenario, the ionization fractions are determined, not from Saha's equation, but from the ratios of ionization and recombination rates.

The local ionization fractions are defined as ratios of number densities for individual atom species,

$$
\begin{aligned}
& \chi_{\hat{\mathrm{H}}^{+}}(r)=\frac{n_{\hat{\mathrm{H}}^{+}}}{n_{\hat{\mathrm{H}}^{0}}+n_{\hat{\mathrm{H}}^{+}}} \\
& \chi_{\hat{\mathrm{He}}^{+}}(r)=\frac{n_{\hat{\mathrm{He}}^{+}}}{n_{\hat{\mathrm{He}}{ }^{0}}+n_{\hat{\mathrm{He}}^{+}}+n_{\hat{\mathrm{He}}}{ }^{++}} \\
& \chi_{\hat{\mathrm{He}}}{ }^{++}(r)=\frac{n_{\hat{\mathrm{He}}}{ }^{++}}{n_{\hat{\mathrm{He}} \mathrm{e}^{0}}+n_{\hat{\mathrm{He}}}{ }^{+}+n_{\hat{\mathrm{He}}}{ }^{++}} .
\end{aligned}
$$

\footnotetext{
${ }^{3}$ Small differences arise due to the increased ionization energies corresponding to higher values of $\hat{v} / v$, resulting in slightly lower temperatures for higher values of the mirror Higgs vev. However, this effect is too minor to affect our discussion.

${ }^{4}$ If this were not the case, the dependence of the mirror plasma heat capacity on ionization would have to be taken into account.
} 
Solving the equation $d n_{\hat{\mathrm{H}}^{+}} / d t=0$, assuming no photoionization and neglecting doubleionization and double-recombination processes yields,

$$
\chi_{\hat{\mathrm{H}}^{+}}=\frac{\left\langle\sigma_{\text {ion }\left(\hat{\mathrm{H}}^{0}\right)} v\right\rangle}{\left\langle\sigma_{\operatorname{ion}\left(\hat{\mathrm{H}}^{0}\right)} v\right\rangle+\left\langle\sigma_{\operatorname{rec}\left(\hat{\mathrm{H}}^{+}\right)} v\right\rangle},
$$

where $\sigma_{\operatorname{ion}\left(\hat{\mathrm{H}}^{0}\right)}$ and $\sigma_{\operatorname{rec}\left(\hat{\mathrm{H}}^{+}\right)}$are the relevant thermally averaged ionization and recombination cross sections. Similarly, solving $d n_{\hat{\mathrm{He}}}{ }^{+} / d t=d n_{\hat{\mathrm{He}}^{++}} / d t=0$ for helium leads to,

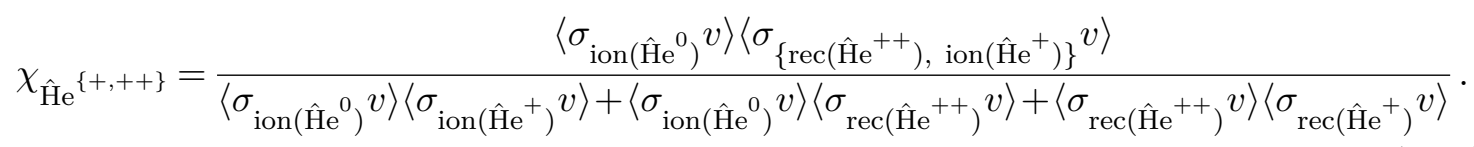

The averaged cross sections $\langle\sigma v\rangle$ are computed assuming a thermal distribution of the initial state electrons and neglecting the motion of the relatively slow atoms. Ref. [91] recently summarized the various ionization, recombination and cooling cross sections and rates for dissipative DM with one nucleus-like and one electron-like constituent. These expressions can be directly applied to mirror hydrogen, with the mirror Rydberg energy given by $\operatorname{Ryd}=(13.54 \mathrm{eV}) \times(\hat{v} / v)$. For mirror helium, it is a reasonable approximation to treat the participating electron as if it were bound in a hydrogen-like atom with some effective charge $Z_{\text {eff }}$. We therefore employ the same expressions for the cross sections as for the hydrogen-like atom, but with the substitution $\mathrm{Ryd}=(24.48 \mathrm{eV}) \times(\hat{v} / v)$ for $\mathrm{He}^{0}$ and $\operatorname{Ryd}=(54.17 \mathrm{eV}) \times(\hat{v} / v)$ for $\mathrm{He}^{+}$. For the isothermal profile in the optically thin regime, ionization is trivially constant throughout the halo, since the thermally averaged cross sections do not depend on density.

We discuss the optical depth of the mirror halo in section 3.2.2 to verify that Rydberg energy photons escape and hence detailed balance does not apply. This justifies our use of ionization and recombination cross section ratios to determine the ionization fractions $\chi_{i}$. These cross sections also give ionization and recombination timescales for the different mirror atomic species, shown as magenta and cyan lines in figures 2 and 3 . The high degree of ionization in the halo is reflected by the very short ionization timescale compared to the other timescales in the system (including the recombination timescale) in regions where most of the mirror matter is concentrated.

\subsection{Mirror baryon cooling}

We now discuss various cooling mechanisms that can cause the mirror halo to loose pressure support and collapse, potentially leading to disk formation. This would have a dramatic effect on the prospects for direct and indirect detection of the twin subcomponent of DM [116-119]. Cooling occurs through the emission of mirror photons produced in the scattering of mirror particles. In section 3.2.1 we discuss the most important cooling processes, which include bremsstrahlung and various atomic processes such as ionization, recombination and collisional excitation. If the cooling timescale is shorter than the age of the universe, which we take to be 14 Gyr, there is a possibility that the twin particles have 
condensed into a disk. In section 3.2.2 we discuss the optical depth of the mirror halo, and verify that the photons produced in these cooling processes escape from the galaxy.

The cooling timescale $t_{\text {cool }}$ that we evaluate is to be compared to the other two relevant timescales; the current age of the universe $t_{\text {universe }}$ and the dynamical or convection timescale

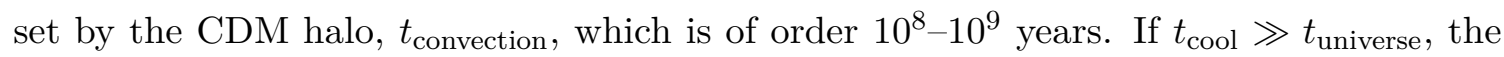
mirror baryon halo has not yet had time to cool significantly since the formation of the Milky Way, and is likely to still be close to its original halo distribution. At the other extreme, if $t_{\text {cool }} \ll t_{\text {convection }}$ within some radius $r<r_{\text {collapse, }}$, the halo will lose pressure support inside that radius and start to undergo catastrophic collapse, which may result in the formation of a disk. The size of such a disk would be expected to be of the same order as $r_{\text {collapse, }}$, but as we discuss in section 3.3 , it is very difficult to extrapolate this result directly to the mirror baryon distribution today. Finally, if $t_{\text {convection }} \ll t_{\text {cool }} \ll t_{\text {universe }}$, the outcome is even more uncertain. The halo initially cools gradually without loss of pressure support. As the temperature drops, the cooling timescale decreases and the halo may eventually reach the aforementioned regime where $t_{\text {cool }} \ll t_{\text {convection }}$ within some radius. Whether this happens depends in part on how efficiently the halo maintains an isothermal profile during the cooling process, since cooling occurs predominantly via photon emission from the inner regions. These complications make quantitative predictions about the mirror baryon distribution today challenging. Nevertheless, our analysis will still reveal important quantitative and qualitative information that serves to illuminate the range of possibilities we must consider for direct detection.

\subsubsection{Cooling timescales}

We first consider the cooling through the emission of massless mirror photons that are produced through Compton scattering of mirror electrons off the background mirror CMB photons, $\hat{e} \hat{\gamma} \rightarrow \hat{e} \hat{\gamma}$, or through bremsstrahlung emission, $\hat{e} \hat{X}_{i} \rightarrow \hat{e} \hat{X}_{i} \hat{\gamma}$, where $\hat{X}_{i}=\left(\hat{\mathrm{H}}^{+}, \hat{\mathrm{He}} \mathrm{e}^{+}, \hat{\mathrm{H}} \mathrm{e}^{++}\right)$. Both processes lead to energy loss of the mirror electron. If the mirror photon escapes the halo without being reabsorbed, and if $\hat{e}$ and $\hat{X}_{i}$ remain in thermal equilibrium, then $\hat{X}_{i}$ and $\hat{e}$ cool adiabatically together.

We first determine if the equilibrium condition is satisfied. The time for $\hat{X}_{i}$ and $\hat{e}$ to reach thermal equilibrium, $t_{e q}^{i}$, has been estimated as [87, 120],

$$
\begin{aligned}
t_{e q}^{i} & \approx \frac{m_{\hat{X}_{i}} m_{\hat{e}}}{2 \sqrt{3 \pi} \hat{\alpha}_{e m}^{2}}\left(\frac{3 T_{\text {iso }}}{m_{\hat{e}}}\right)^{\frac{3}{2}}\left[n_{\hat{e}} \log \left(1+\frac{v_{\hat{e}}^{4} m_{\hat{e}}^{2}}{\hat{\alpha}_{e m}^{2} n_{\hat{e}}^{2 / 3}}\right)\right]^{-1} \\
& \approx 6 \times 10^{7} \mathrm{yr}\left(\frac{m_{\hat{X}_{i}}}{1 \mathrm{GeV}}\right)\left(\frac{T_{\text {iso }}}{100 \mathrm{eV}}\right)^{\frac{3}{2}} \sqrt{\frac{m_{e}}{m_{\hat{e}}}}\left(\frac{10^{-3} \mathrm{~cm}^{-3}}{n_{\hat{e}}}\right)\left[\log \left(1+\frac{9 T_{\mathrm{iso}}^{2}}{\hat{\alpha}_{e m}^{2} n_{\hat{e}}^{2 / 3}}\right)\right]^{-1} .
\end{aligned}
$$

We find that for the parameter range of interest, the characteristic equilibration times for all $\hat{X}_{i}$ are much less than the age of the universe. As we shall see, the time scale of equilibration is also much shorter than the characteristic cooling timescales. 
We can obtain an expression for the ratio of the rate of energy loss per unit volume through Compton cooling to the initial energy density in mirror baryons and electrons [121],

$$
\begin{aligned}
-\frac{d U / d t_{\text {Compton }}}{\frac{3}{2} T_{\text {iso }}\left(\sum n_{\hat{X}_{i}}+n_{\hat{e}}\right)} & \approx \frac{64 \pi^{2} \hat{\alpha}_{e m}^{2}}{135} \frac{n_{\hat{e}}}{\sum n_{\hat{X}_{i}}+n_{\hat{e}}} \frac{\hat{T}^{4}(z)}{m_{\hat{e}}^{3}} \\
& \approx \frac{1}{1.6 \times 10^{13} \mathrm{yr}}\left(\frac{\hat{T}(z)}{4 \mathrm{~K}}\right)^{4}\left(\frac{m_{e}}{m_{\hat{e}}}\right)^{3} .
\end{aligned}
$$

Here $\hat{T}(z)$ denotes the temperature of the mirror CMB photons at redshift $z$. The summation is over the various ions, $\hat{X}=\left(\hat{\mathrm{H}}^{+}, \hat{\mathrm{H}} \mathrm{e}^{+}, \hat{\mathrm{H}}^{2+}\right)$. The ratio of number densities is determined in terms of $\hat{Y}_{p}\left({ }^{4} \hat{\mathrm{He}}\right)$ in the limit that the mirror halo is fully ionized. We find from this that for the relevant range of redshifts, $z \lesssim \mathcal{O}(10)$, the time scale of Compton cooling is greater than the age of the universe, and we do not consider this process further.

Cooling via bremsstrahlung emission is much more efficient. The corresponding time scale is given by [91]

$$
t_{\mathrm{brem}}(r) \approx \frac{3^{5 / 2}}{2^{9 / 2} \sqrt{\pi}} \frac{\sum n_{\hat{X}_{i}}+n_{\hat{e}}}{n_{\hat{e}} \sum Z_{i}^{2} n_{\hat{X}_{i}}} \frac{m_{\hat{e}}^{3 / 2} T_{\text {iso }}^{1 / 2}}{\hat{\alpha}_{e m}^{3}}
$$

(see also $[121,122]$ ). The relevant number densities are obtained from the profiles computed in the previous section. We find that in the parameter range of interest, the timescale associated with cooling through bremsstrahlung emission can be less than the age of the universe in the dense inner regions of the galaxy.

In addition to energy loss via bremsstrahlung, mirror electrons in the halo can also lose energy through atomic processes that involve $\mathcal{O}\left(\right.$ Ryd) energies, such as ionization ${ }^{5}$ $\left(\hat{e}^{-}+\hat{X}_{i} \rightarrow \hat{X}_{i}^{+}+2 \hat{e}^{-}\right)$, recombination $\left(\hat{e}^{-}+\hat{X}_{i}^{+} \rightarrow \hat{X}_{i}+\hat{\gamma}\right)$, and collisional excitation $\left(\hat{e}^{-}+\hat{X}_{i} \rightarrow \hat{e}^{-}+\hat{X}_{i}^{*} \rightarrow \hat{e}^{-}+\hat{X}_{i}+\hat{\gamma}\right)$. For all these processes, recent estimates of the relevant cross sections and energy loss rates can be found in [91], and we adapt them for use with singly- or doubly-ionized helium as described in section 3.1.4. We find that in the inner regions of the galaxy, the cooling timescale from atomic processes can be less than the age of the universe. In principle molecular cooling processes can also play a role [92], but given the high degree of ionization in the mirror halo, we neglect them in our simple analysis.

Based on this discussion, we can make some observations about the dependence of the cooling rate on the parameters of the MTH.

- We find that the dependence of $t_{\text {cool }}$ on the electroweak VEV in the twin sector is quite modest in the $\hat{v} / v \sim 1-5$ range of interest. Near the SM value of 1 , bremsstrahlung cooling dominates. For MTH-like values of $3-5$, the larger mirror electron mass and ionization energies give rise to a small but important neutral atom population, which makes collisional cooling processes dominant. This compensating behavior explains the insensitivity of cooling time scales on the (mirror-) Higgs vev, and at our level of precision $\hat{v} / v$ is not important to our discussion.

\footnotetext{
${ }^{5}$ The collisional ionization comes from a free electron impact that ionizes a formerly bound electron, taking energy from the free electron. The temperature of the particles is therefore lower after the ionization.
} 
- The cooling time scale scales inversely with the number density of mirror particles, $t_{\text {cool }} \sim r_{\text {all }}^{-1}$, so that lower mirror baryon densities are associated with slower cooling. This is because the cooling processes arise from the collisions of mirror particles, and their rates go down if the number density of mirror particles is reduced.

- Cooling depends nontrivially on the mirror helium fraction $\hat{Y}_{p}\left({ }^{4} \hat{\mathrm{He}}\right)$, with the SM-like value of $\hat{Y}_{p}\left({ }^{4} \hat{\mathrm{He}}\right)$ being near-optimal for cooling, and other values near 0 or 1 cooling much less efficiently. This can be traced back to our finding that SM-like values of $\hat{Y}_{p}\left({ }^{4} \hat{H e}\right)$ lead to the most tightly packed profiles.

Since the SM particles are much more abundant than their mirror counterparts, and because $\hat{Y}_{p}\left({ }^{4} \hat{\mathrm{He}}\right)$ is near the optimum value for cooling, we can immediately conclude that mirror baryons cool much slower than the SM baryons. However, determining how the cooling rate compares to the convection timescale requires explicit calculation for each profile. We discuss these results in section 3.2.3.

\subsubsection{Optical depth}

In the discussion of cooling and ionization above, we assumed the mirror halo was optically thin, so that mirror photons produced in the various cooling processes escape the galaxy without being recaptured. We now justify this assumption. The two most important processes that lead to absorption or scattering of mirror photons in the halo are Thomson scattering, $\hat{e}^{-} \hat{\gamma} \rightarrow \hat{e}^{-} \hat{\gamma}$, and photoionization, $\hat{X}_{i} \hat{\gamma} \rightarrow \hat{X}_{i}^{+} \hat{e}^{-}$. The mean free path of a photon with respect to Thomson scattering is given by,

$$
\ell_{T}=\frac{1}{\sigma_{T} n_{\hat{e}}}=\frac{3 m_{\hat{e}}^{2}}{8 \pi \hat{\alpha}_{e m}^{2} n_{\hat{e}}}
$$

The corresponding expression for photoionization takes the form,

$$
\ell_{\hat{X}}=\frac{1}{\sigma_{\operatorname{photo}_{\hat{X}} n_{\hat{X}}}},
$$

where $\hat{X}=\hat{\mathrm{H}}^{0}, \hat{\mathrm{H}} \mathrm{e}^{0,+}$. The photoionization cross section is given by [91],

$$
\sigma_{\operatorname{photo}_{\hat{X}}}(\omega)=\frac{2^{5} \pi^{2} \hat{\alpha}_{e m}^{7} m_{\hat{e}}^{2}}{3 \omega^{4}} \frac{e^{-4(\arctan \tau) / \tau}}{1-e^{-2 \pi / \tau}}
$$

where $\omega$ is the mirror photon energy and $\tau \equiv\left(\omega / \omega_{0}-1\right)^{1 / 2}$, where $\omega_{0}$ is the equivalent of the Rydberg energy for the atom, as discussed in section 3.1.4.

The mean free path for photoionization, $\ell_{\hat{X}}$, is much larger than the size of the galaxy. This allows us to neglect photoionization for the remainder of the discussion. The optical depth from Thomson scattering, however, can be smaller than the halo size in the dense inner regions. However, this does not significantly impede the cooling efficiency. The average fractional energy loss of a mirror photon with energy $\omega$ scattering once with a mirror electron at rest (a good approximation since $\omega$ is typically of order $T$ or of order Ryd, 

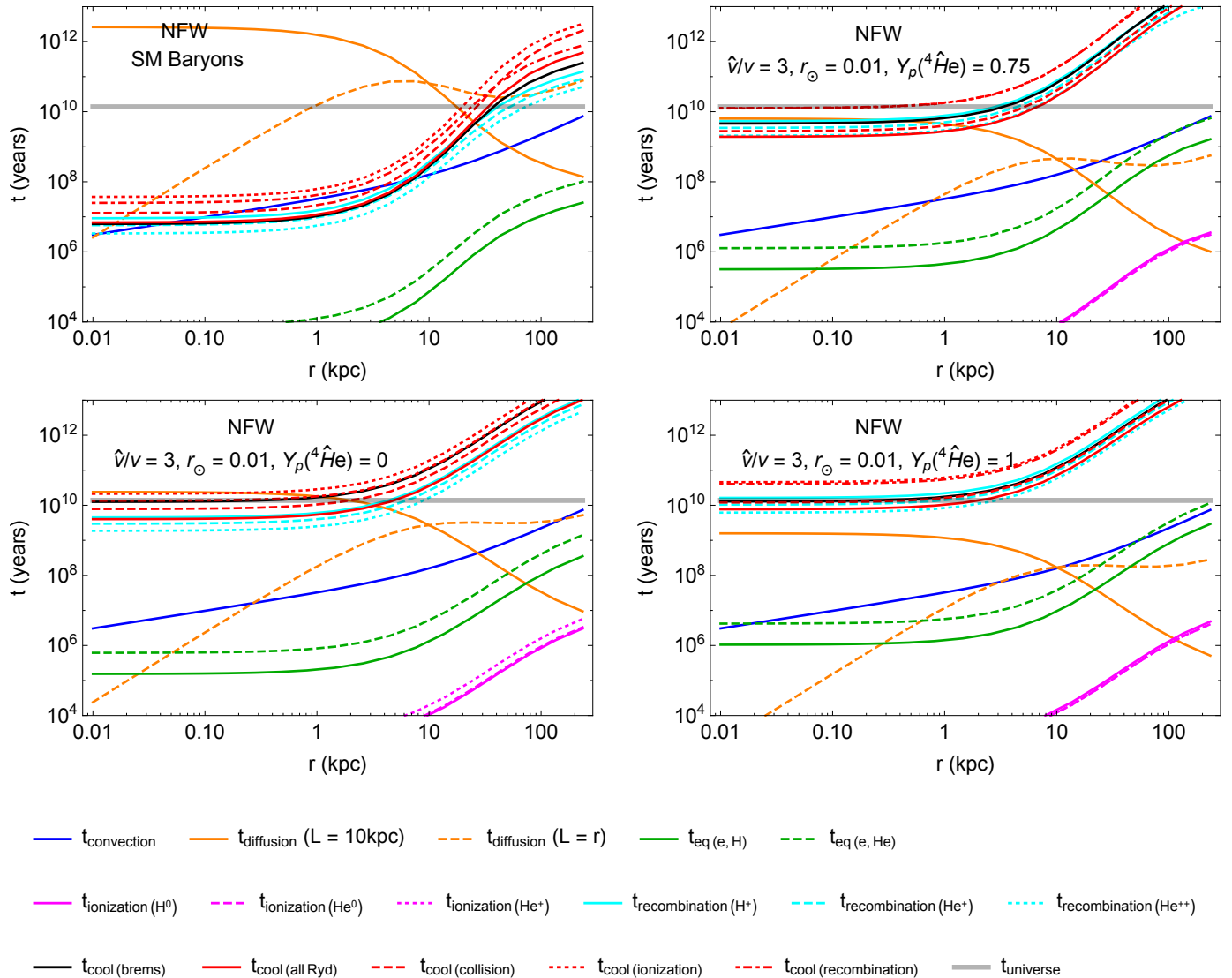

Figure 2. Timescales in the mirror halo for equilibration between mirror electrons and atoms (green), ionization (magenta), recombination (cyan), as well as bremsstrahlung cooling (black) and collision, ionization and recombination cooling (solid red total, various dashings individual), compared to the convection timescale dictated by the CDM halo (blue) and the age of the universe (thick grey line). The diffusion timescale over two length scales $(L=r$ and $L=10 \mathrm{kpc}$ ) is also shown (orange), but this scale is not required to be small for thermal equilibrium in the already isothermal halo. The SM-like isothermal comparison halo is compared to our three MTH benchmark points from table 2 assuming an NFW CDM profile.

both of which are much smaller than $\left.m_{\hat{e}}\right)$ is $\omega / m_{\hat{e}}$. After $n$ scatters, the average energy of a mirror photon that began with initial energy $\omega_{0}$ is given by,

$$
\omega_{n} \approx \frac{m_{\hat{e}} \omega_{0}}{m_{\hat{e}}+n \omega_{0}}
$$

The number of times the photon scatters before traveling the distance $d$ required to leave the halo can be estimated from a random walk, $d \sim \sqrt{n} \ell_{T}$, assuming the photon has an initial energy $\sim T \lesssim$ Ryd.

We find that for the range of MTH parameters of interest, $\hat{v} / v \geq 3$ and $r_{\text {all }} \leq 0.1$, the mirror photons lose very little of their energy before leaving the halo. This is true for both the NFW and Burkert CDM profiles. Therefore, this has no significant effect on our discussion of cooling. The mirror halo is therefore optically thin, justifying our derivation of the ionization fractions in section 3.1.4. 

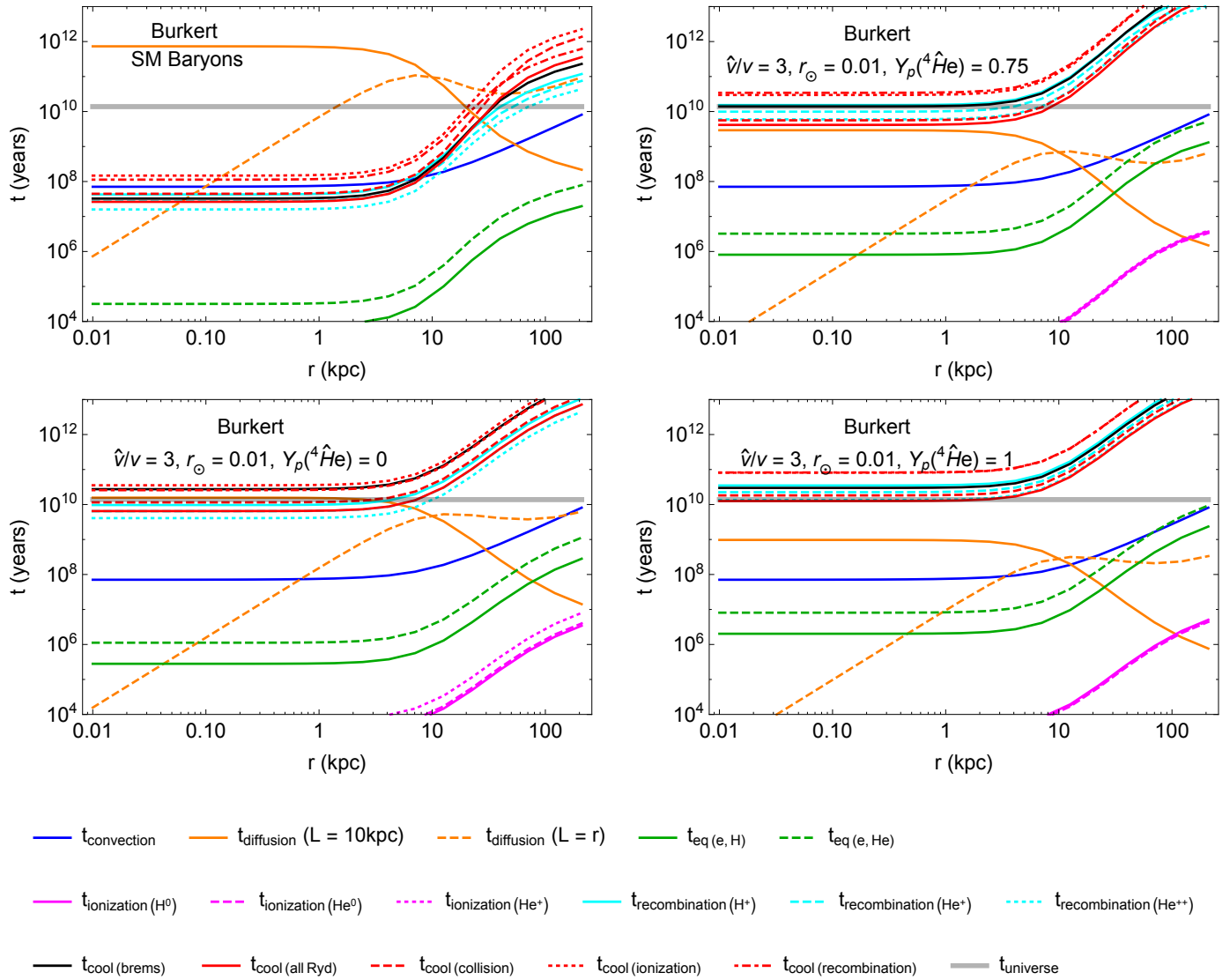

Figure 3. Same as figure 2, but assuming the Burkert CDM profile.

Even for SM-like densities, the attenuation is at most $\sim 10 \%$ for photons emerging from the innermost regions of the halo, which does not significantly affect the cooling timescales. We also find that the attenuation of photons leaving the galaxy remains insignificant if the isothermal halo temperature is lower by a factor of a few compared to $T_{\text {iso }}$, the initial temperature of the adiabatic halo. Therefore, the halo continues to remain optically thin even as cooling progresses.

\subsubsection{Results}

Figures 2 and 3 show the cooling rates from bremsstrahling and $\mathcal{O}$ (Ryd) processes (collision, ionization and recombination) as a function of distance from the galactic center for the NFW and Burkert profiles. These cooling rates are to be compared to the convection timescale and to the age of the universe.

We begin by noting that the SM-like profile cools much faster than the mirror benchmark scenarios. This is primarily due to the fact that $\hat{Y}_{p}\left({ }^{4} \hat{\mathrm{He}}\right)=0.25$, the near-optimal value for cooling, and also the relatively high density compared to our $r_{\text {all }}=0.01 \mathrm{MTH}$ benchmarks. The fastest cooling timescale $t_{\mathrm{cool}}=t_{\text {cool(collision) }}$ drops below the convection timescale for $r<r_{\text {collapse }} \approx 10 \mathrm{kpc}$. Baryons within this radius lose pressure support and start collapsing. The size of $r_{\text {collapse }}$ is roughly consistent with the observed size of the Milky Way visible disk. 
The mirror baryon profiles with $r_{\text {all }}=0.01$ all cool significantly less efficiently than the SM profile, owing to their lower density and different value of $\hat{Y}_{p}\left({ }^{4} \hat{\mathrm{He}}\right)$. Initially, the cooling timescale is much longer than the convection timescale, but still lower than (or close to) the age of the universe. This means that the mirror halo cools gradually without loss of pressure support. However, if the average temperature in the entire halo (or just the inner $10 \mathrm{kpc}$ or so) drops by a factor of $\sim 2$ compared to its initial value, the cooling time scale in the inner region will drop below the convection timescale for $r<r_{\text {collapse }} \sim$ few kpc. We have determined this via direct computation of the isothermal profile at various temperatures. This suggests that if the mirror halo eventually collapses, $r_{\text {collapse }}$ would only be about half as large as the corresponding value for the SM profile. This leads us to posit that if the mirror baryons do form a disk, it is likely to be significantly smaller and younger than the visible baryonic disk of the Milky Way.

The cooling time scale scales inversely with the number density of mirror particles,

$t_{\text {cool }} \sim r_{\text {all }}^{-1}$, so that lower mirror baryon densities are associated with slower cooling. This means that if the mirror baryon mass fraction $r_{\text {all }}$ lies below some critical value, the mirror particles in the Milky Way do not undergo significant cooling, so that their distribution today remains in the form of an ionized halo. With our assumptions, we find this critical value to be $r_{\text {all }} \sim 10^{-2}$. However, varying $T_{\text {iso }}$ by a factor of 2 in either direction alters the critical value of $r_{\text {all }}$ by 2 orders of magnitude, and so there are large uncertainties.

These observations hold for both NFW and Burkert CDM halos. It seems clear that although cooling is significantly less effective in the mirror halo than for the SM, there is still the possibility of forming a dark disk. However, extrapolating these results to the mirror baryon distribution today is not simple. We discuss this in the next section.

\subsection{Resulting mirror baryon distribution today}

The above analysis gives some idea of how a mirror matter component in our Milky Way might have behaved during the early stages of galaxy formation. This simple approach can yield useful quantitative information in the limit that the cooling rate is slow compared to the lifetime of the universe, or if complicated astrophysical processes can be neglected, as would be the case in a dissipative DM model that does not possess an analogue of nuclear physics [92]. In the MTH model with very small mirror DM fraction $r_{\text {all }} \lesssim 10^{-2}$, the cooling rate is so low that the mirror matter distribution would still be close to its original isothermal profile today. Unfortunately, for larger mirror DM fractions, cooling is significant and we expect that feedback from astrophysical processes in the mirror sector cannot be neglected. In this case, our only firm conclusion is that it is not possible to quantitatively predict the mirror matter distribution at the present time. Even so, we can organize the possible outcomes for the distribution of the mirror component in a useful manner. This will enable us to study the prospects for direct detection in section 4 .

Naively, sufficient cooling in the halo should lead to the formation of an accretion disk. We have shown that cooling in the mirror sector, while significantly less efficient than in the visible sector, can still lead to a loss of pressure support. The existence of the visible disk implies sufficient angular momentum for disk formation, and also a relatively quiet merger history so that the creation of a dark disk would not be disrupted. However, in 
the visible sector, disk formation cannot be quantitatively reproduced without detailed magnetohydrodynamic (MHD) $N$-body simulations [103-107]. The size of the disk and the density profile of the SM baryons depend sensitively on astrophysical feedback processes.

The mirror sector in the MTH model, although similar to the SM in many respects, is expected to have its own version of nuclear physics. Mirror protons and neutrons will form elements up to mirror helium [62] and possibly heavier elements as well, but obtaining precise predictions about nuclear spectra, binding energies and reaction rates is extremely difficult. While it therefore seems quite likely that "mirror stars" [123-127] would form, possibly giving rise to spectacular observational signatures [66-69], their distribution and characteristics, challenging to predict even if the microphysics were fully understood, is presently unknown. The details of mirror-baryonic feedback are therefore expected to be very different from the SM, making it very challenging to perform reliable MHD $N$-body simulations of the mirror sector. ${ }^{6}$ For appreciable DM fractions $r_{\text {all }} \gtrsim 10^{-2}$, we therefore have to consider a range of possibilities for how the mirror matter distribution could look like today.

Our aim is to parametrize the possible distributions of mirror matter in our stellar neighborhood. This will be the most useful approach when considering direct detection signatures in the next section. To this end, we define the parameter,

$$
r_{\odot}=\left.\frac{\rho_{\text {mirror }}\left(R_{\odot}\right)}{\rho_{\mathrm{CDM}}\left(R_{\odot}\right)}\right|_{\text {today }}
$$

which parametrizes the fractional contribution of mirror matter to the total density of DM in our local neighborhood. Below, we define two benchmark local mirror matter distributions, one optimistic and one pessimistic for direct detection, which bracket the range of possibilities and allow us to make quantitative statements about the prospects for discovering mirror matter. For each benchmark, we look to express the direct detection limits in terms of a bound on $r_{\odot}$.

1. Halo-like. This assumes that mirror matter either

(a) does not collapse into a disk due to inefficient cooling, or

(b) does not collapse into a disk due to strong heating processes that keep the halo hot, or

(c) does collapse into a disk, but that the Sun is outside of the disk radius. The outer portions of the mirror matter distribution could still have a diffuse halo-like form, which has an analogue in the SM. Star formation causes the Milky Way disk to be baryon-depleted, but recently those missing baryons have been discovered at large distances from the center of the Milky Way [100], (see also [130]).

To represent this possibility, we choose a benchmark mirror DM distribution that is essentially that of conventional CDM, with local velocity dispersion $v_{\odot} \approx 220 \mathrm{~km} / \mathrm{s}$,

\footnotetext{
${ }^{6}$ This is in marked contrast to mirror DM models with an exact $\mathbb{Z}_{2}$ symmetry $[77,80,81,128]$, for which SM astrophysics can be more directly applied to the dark sector. Dissipative DM was recently studied in simulations [129], but in a very different scenario featuring two nearly degenerate DM states, and without the kinds of feedback processes that arise from mirror star formation.
} 
the same as that of the CDM halo at the Earth's location [131]. This is not exactly the same as the velocity dispersion obtained from the local temperature in our isothermal mirror halo solutions (see table 2), but we choose the standard CDM value of $v_{\odot}=v_{0}$ for ease of reach comparison with other DM scenarios. Unlike CDM, we do not cut off the distribution at the galactic escape velocity $v_{\mathrm{esc}}^{\mathrm{gal}} \approx 544 \mathrm{~km} / \mathrm{s}$ in the galactic frame [132], since mirror matter is not collisionless. The velocity of the Earth relative to the halo is taken to be $v_{\mathrm{E}} \approx 233 \mathrm{~km} / \mathrm{s}$. The local mirror matter density is not fixed, but it seems reasonable that $r_{\odot}$ is of the same order as the cosmological value $r_{\text {all }}$. We consider the limiting cases that the local distribution of mirror baryons is either fully ionized or fully neutral, though the former is more likely if the mirror matter is in a hot halo.

2. Disk-like. This assumes that the mirror matter collapses into a disk, and that the Sun is inside that disk. Assuming that the dark disk is spatially aligned with our own, a pessimistic assumption is that the DM velocity dispersion is the same as that of stars and gas in our local stellar neighborhood, leading to $v_{\odot}=20 \mathrm{~km} / \mathrm{s}[131,133]$. Assuming no relative motion of the dark disk with respect to the Sun, the only motion of the Earth relative to the mirror matter would be due to the Earth's rotation around the Sun with velocity $v_{\mathrm{E}}=30 \mathrm{~km} / \mathrm{s}$. The local DM density is very difficult to estimate. On the one hand, collapse into a disk could concentrate the DM and could lead to $r_{\odot} \gg r_{\text {all }}$. On the other hand, mirror star formation and shedding of angular momentum during disk formation could deplete free mirror baryons from the disk. We will state direct detection constraints in terms of the unknown $r_{\odot}$ in the next section. However, as long as the local DM fraction is within two orders of magnitude of the cosmic average value, the direct detection bounds on the couplings of DM to the SM derived under the assumption $r_{\odot}=r_{\text {all }}$ will be accurate at the level of an order of magnitude. Just as for the halo, we will consider two possibilities for local ionization, either fully ionized or fully neutral.

For simplicity we assume that even in the disk-like configuration, the local mirror matter distribution is approximately thermal. Of course, local mirror astrophysics could violate this assumption while introducing correlations between local ionization and temperature (which is certainly the case in the SM). However, the naive assumption of a thermal distribution, while also allowing for various possible states of ionization, will be sufficient to demonstrate the range of possible outcomes for direct detection.

Studies of star motion in the Milky Way can be used to constrain the amount of dissipative DM that forms a disk [101, 134, 135]. Recently, results from the Gaia survey $[134,135]$ have set an upper bound on disk DM to be at most a few percent of DM in the Milky Way. This limit assumes that the dark disk has thickness less than about $100 \mathrm{pc}$ and exhibits a radial profile similar to the SM disk. It also assumes that most of the DM component of which the disk is composed resides inside the disk. If the disk is thicker than about $200 \mathrm{pc}$, the GAIA constraints lose sensitivity. In our mirror sector, we do not know the radial profile or the height of the disk, 
if any. ${ }^{7}$ Furthermore, even if $r_{\text {all }}=1 \%$, it is unlikely that all of the mirror matter would end up in the disk, just as all the SM baryons do not end up in the visible disk. Therefore the Gaia constraints do not impose a robust constraint on $r_{\text {all }}$, or even on $r_{\odot}$, the fractional contribution of mirror matter to the local DM density, in the MTH framework.

3. Nothing in our neighborhood. One could in principle imagine that the entire mirror halo collapses into the center of the galaxy, and that no appreciable abundance is left in our stellar neighborhood. However, the fact that a significant fraction of SM baryons reside outside the disk makes this a somewhat implausible scenario. Nevertheless, it is worth keeping in mind that even a local mirror matter abundance that is significantly reduced compared to the galactic average could still lead to a direct detection signal.

Even so, it is amusing to consider that the central black hole of our Milky Way has a mass of $\sim 10^{6} M_{\odot}$, which is of order $10^{-6}$ of the mass of the full DM halo. Given the approximate nature of our cooling estimates, one might envisage the possibility that the central black hole was formed from a mirror matter halo that collapsed relatively slowly and adiabatically within about a billion years or so, without significant mirror star formation or loss of pressure support. (A subdominant dissipative DM component that could seed central black hole production via gravithermal collapse has previously been considered, for example in [94, 136-138].) However, it is far beyond the scope of this paper to carefully examine this possibility.

With the maximally pessimistic option (3) being somewhat unlikely, the information at our disposal does not allow us to say whether the halo-like option (1) or the disk-like option (2) is more favored. We therefore examine direct detection in both of these scenarios, noting that they represent an optimistic and pessimistic scenario respectively from the point of view of direct detection rates. We expect that the true direct detection prospects are likely to lie somewhere between these two extremes.

\section{Direct detection of sub-nano-charged mirror matter}

Current cosmological constraints allow up to about $10 \%$ of the DM density to consist of mirror matter in the MTH framework [62]. In the near future, improved measurements of large scale structure are expected to be able to constrain this fraction to the sub-percent level. In this section we discuss how an even more subdominant component can still give rise to distinctive signals in direct detection experiments, which may allow this class of theories to be distinguished from other models.

Given the large uncertainties involved in the cooling of the halo discussed in the previous section, we focus on the limiting cases of a mirror DM distribution that is either halo-like or disk-like, and either fully ionized or fully atomic. Our analysis finds that by combining the results of different direct detection experiments, it may be possible to differentiate between

\footnotetext{
${ }^{7}$ One might try and estimate the disk height as was done in [87], but this is not appropriate given the likely importance of feedback in our mirror sector.
} 
these different possibilities for the mirror matter distribution and ionization. Furthermore, it may be possible to discern the multi-component nature of mirror DM, and thereby distinguish this class of models from other theories.

In the MTH, the SM and mirror sectors interact through the Higgs portal. Mirror DM can therefore scatter off SM particles through Higgs exchange. Unfortunately, this interaction is far too small to allow for direct detection of mirror hydrogen or mirror helium nuclei. This can be easily seen by comparison to the FTH scenario [43], in which the mirror tau is a potential candidate for weakly interacting massive particle (WIMP) DM [51, 52]. For masses below $10 \mathrm{GeV}$, the mirror tau-nucleon direct detection cross section is $\lesssim 10^{-45} \mathrm{~cm}^{2}$, which is below the neutrino floor and already very challenging to detect. The coupling of mirror protons to the Higgs is an order of magnitude smaller than that of the mirror tau. This, together with the lower mirror baryon relic density, makes it clear that Higgs exchange is not expected to generate an observable direct detection signal in the foreseeable future. Similarly, possible contributions to the scattering via scalar twin-bottomonium exchange [53] do not increase the cross section above the neutrino floor.

The only other renormalizable interaction between the two sectors allowed by the gauge symmetries is a kinetic mixing term between the hypercharge gauge boson of the SM and its mirror counterpart,

$$
\frac{\epsilon}{2 \cos \theta_{W}} B_{\mu \nu} B^{\prime \mu \nu}
$$

At low energies this leads to kinetic mixing between the SM photon and its mirror counterpart (for a review, see e.g. [139]). Since both U(1) gauge groups are unbroken, mirror baryons acquire an electric charge proportional to $\epsilon$, and can be detected in electron recoil (ER) and nuclear recoil (NR) direct detection experiments through photon exchange.

It is crucial for the viability of the MTH framework that the hidden and visible sectors remain out of equilibrium with each other after asymmetric reheating has taken place. Avoiding recoupling of the mirror sector at temperatures of order a few $\mathrm{MeV}$ via eê scattering leads to an upper bound on the kinetic mixing parameter [63],

$$
\epsilon \lesssim 10^{-9}
$$

In the MTH model, no kinetic mixing is generated through 3-loop order [1], and therefore even such small values of $\epsilon$ are radiatively stable. Therefore, this bound can naturally be satisfied provided that the contributions to $\epsilon$ from UV physics are also small ${ }^{8}$.

In general, the size of $\epsilon$ depends on details of the UV completion of the MTH model, but in the asymmetrically reheated scenario, it has to satisfy eq. (4.2). Encouragingly, gravity-mediated interactions between the two sectors at the Planck scale are expected to generate $\epsilon \sim 10^{-13}$ [64]. DM with a tiny electric charge, of order $10^{-9}$ or less, may therefore constitute a key feature of MTH models. Such sub-nano-charged DM cannot be probed at colliders or fixed-target experiments [139], but provides a natural sensitivity goal

\footnotetext{
${ }^{8}$ There is also a constraint on $\epsilon$ arising from the distortions in the CMB that result from energy transfer between the two sectors at temperatures below $\mathcal{O}(100) \mathrm{eV}$, through scattering of the residual $e$ and $\hat{e}$. However, this effect is suppressed by the temperature asymmetry between the SM and mirror sectors, resulting in a weaker bound on $\epsilon$ than the one in eq. (4.2).
} 
for direct detection experiments, in particular if the expected size of the gravity-mediated contributions to $\epsilon$ is realized.

It is worth emphasizing that several constraints that have been applied to millicharged DM in the past are not applicable to the MTH scenario. It has been argued that any subcomponent of DM with a detectable electric charge will be expelled from the disk by galactic magnetic fields $[140,141]$, and so cannot give rise to a direct detection signal. However, this argument does not apply in the case of the MTH because, just like SM ions, the twin ions radiate and interact with each other with large cross sections and quickly dissipate the energy they obtain from the magnetic field. ${ }^{9}$ DM carrying a sizable unbroken dark charge might also be expected to be severely constrained by Bullet Cluster measurements, since the long-range Coulomb interaction induces instabilities in the DM plasma [143]. However, the small DM mass fractions we consider, $r_{\text {all }} \lesssim 10 \%$, means the MTH framework is not affected by these constraints.

\subsection{Local mirror baryon ionization and velocity distribution}

We will follow the road map laid out in section 3.3 to study the prospects for direct detection of mirror DM in the MTH framework. We focus on the limiting cases when the mirror matter is either in a halo-like distribution or has collapsed into a disk. The local mirror DM fraction $r_{\odot}$, defined as per eq. (3.26), is the parameter that direct detection searches constrain or measure. For the local DM density we take $\rho_{\mathrm{CDM}}\left(R_{\odot}\right) \approx 0.3 \mathrm{GeV} / \mathrm{cm}^{3}$. Note that this is slightly different from the value of $\rho_{\mathrm{CDM}}\left(R_{\odot}\right) \approx 0.5 \mathrm{GeV} / \mathrm{cm}^{3}$ assumed in the simulated CDM profiles used in the previous section. The value of $0.3 \mathrm{GeV} / \mathrm{cm}$ is used for ease of comparison with various existing direct detection limits, but its precise value does not meaningfully affect our discussion.

The signal also depends on the local mirror helium mass fraction $\hat{Y}_{\odot}$. Note that, in general, this can be very different from the cosmic value of the helium fraction $\hat{Y}_{p}\left({ }^{4} \hat{\mathrm{He}}\right)$ (for $\left.\hat{Y}_{p}\left({ }^{4} \hat{\mathrm{He}}\right) \neq 0,1\right)$, as can be seen in table 2 . It is hence necessary to consider different possibilities for $\hat{Y}_{\odot}$ even if the cosmic value of $\hat{Y}_{p}\left({ }^{4} \hat{\mathrm{He}}\right)$ is close to the asymmetrically reheated MTH expectation of $\sim 0.75$. Therefore, we consider three benchmark values for our sensitivity estimates; $\hat{Y}_{\odot}=0,1$, and 0.75 . For each case, we derive sensitivities for the local $r_{\odot}$ in the natural range of MTH parameters, $\hat{v} / v \sim 3-5$.

We focus on the limiting cases that the mirror matter in our local neighborhood is either fully ionized or fully atomic. Intermediate ionizations, although possible, do not significantly change our conclusions regarding the range of sensitivity. The mass fractions of mirror hydrogen and helium in our local neighborhood are

$$
r_{\hat{\mathrm{H}}, \odot}=\left(1-\hat{Y}_{\odot}\right) r_{\odot}, \quad r_{\hat{\mathrm{He}}, \odot}=\hat{Y}_{\odot} r_{\odot}
$$

In the limit of complete ionization the mass fraction contributed by free mirror electrons is given by,

$$
r_{\hat{e}, \odot}=r_{\odot}\left(\frac{m_{\hat{e}}}{m_{\hat{\mathrm{H}}}}\right)\left[1-\frac{\hat{Y}_{\odot}}{2}\right] .
$$

\footnotetext{
${ }^{9}$ From eq. (3.2) of [140], the relaxation time of twin baryon scattering is only of order 100 years, which is much shorter than the time scale $\tau_{a c c}$ for the momentum increase due to the galactic magnetic field. Also see the discussion in ref. [142]
} 
The direct detection signal from mirror matter can then be determined once the local mirror baryon velocity dispersion $v_{\odot}$ and the velocity of the Earth relative to the mirror baryons $v_{\mathrm{E}}$ are specified. Since, in general, mirror particles scatter many times with each other before they have a chance to escape the galaxy, this distribution is not cut off at the galactic escape velocity $v_{\text {esc }}$.

An important feature of multi-component DM whose subcomponents are in thermal equilibrium with each other is that the individual components $\hat{\mathrm{H}}, \hat{\mathrm{H}} \mathrm{e}$ and $\hat{e}$ have very different velocity dispersions that depend on their masses,

$$
v_{\odot i}=v_{\odot} \sqrt{\frac{\bar{m}_{\odot}}{m_{i}}}=v_{\odot} \sqrt{\frac{\bar{m}_{\odot}}{m_{\hat{\mathrm{H}}}}} \sqrt{\frac{m_{\hat{\mathrm{H}}}}{m_{i}}}, \quad i=\hat{\mathrm{H}}, \hat{\mathrm{H}} \mathrm{e}, \hat{e} .
$$

Here $\bar{m}_{\odot} \equiv \bar{m}\left(R_{\odot}\right) \sim \mathcal{O}\left(m_{\hat{\mathrm{H}}}\right)$ is the average mass per mirror particle near the location of the Earth, and is fully determined in terms of $\hat{Y}_{\odot}$ and the local ionization. For the $\hat{Y}_{\odot}=0.75$ benchmark the mirror hydrogen velocity dispersion is close to the standard $v_{\odot}$, but only half that big for mirror helium and enhanced by a factor of 20-30 for free mirror electrons (if present). As we shall see, these fast mirror electrons are a promising target for direct detection.

With these ideas in mind we now specify the four scenarios for which we will determine the direct detection signal.

1. Ionized halo: $\chi_{\hat{\mathrm{H}}^{+}}=\chi_{\hat{\mathrm{H}}^{++}}=1, v_{\odot}=220 \mathrm{~km} / \mathrm{s}, v_{\mathrm{E}}=233 \mathrm{~km} / \mathrm{s}$.

In this scenario, mirror electrons will be very fast, $v_{0 \hat{e}} \sim 6000 \mathrm{~km} / \mathrm{s}$, and can lead to spectacular signals at ER detectors. Mirror hydrogen and helium can both show up in ER and NR detectors, and can potentially be distinguished from each other.

2. Ionized disk: $\chi_{\hat{\mathrm{H}}^{+}}=\chi_{\hat{\mathrm{He}}^{++}}=1, v_{\odot}=20 \mathrm{~km} / \mathrm{s}, v_{\mathrm{E}}=30 \mathrm{~km} / \mathrm{s}$.

This case is similar to the halo, but with much lower $v_{\odot}$ and $v_{\mathrm{E}}$. As a result, all recoil energies are reduced, and mirror baryons become invisible to NR detectors. Mirror electrons now have a velocity distribution similar to that of standard CDM in a halo. While mirror electrons can still be detected in ER experiments, ER detection of the slow mirror nuclei would require detectors with much lower threshold than what is likely to be available in the near future.

3. Atomic halo: $\chi_{i}=0, v_{\odot}=220 \mathrm{~km} / \mathrm{s}, v_{\mathrm{E}}=233 \mathrm{~km} / \mathrm{s}$.

As compared to the ionized halo, the velocity dispersions of the mirror baryons are only slightly different (due to the absence of free electrons), and so NR detection of $\hat{\mathrm{H}}, \hat{\mathrm{H} e}$ proceeds almost identically. ER detection of mirror baryons is suppressed by a mirror atomic form factor, and there is no separate signal from mirror electrons. Note that a mirror matter distribution that survives in the halo-like state until today is expected to be very hot and therefore fully ionized. Although we believe that this distribution is rather unlikely, we include it for completeness. 
4. Atomic disk: $\chi_{i}=0, v_{\odot}=20 \mathrm{~km} / \mathrm{s}, v_{\mathrm{E}}=30 \mathrm{~km} / \mathrm{s}$.

A very challenging scenario without NR signals (just as in the case of the ionized disk), and also without an ER signal from free electrons. Mirror baryons only show up in ER detectors with very low recoil, with a rate that is further suppressed by the mirror atomic form factor. Direct detection of this scenario may require ultra-low-threshold ER experiments, and may not be possible in the near future.

We see that the ionized halo offers the most promise for direct detection, while the atomic disk is by far the most pessimistic. The true sensitivity is expected to lie somewhere between these different limiting cases. Fortunately, the sensitivity of direct detection is highly complementary to astrophysical probes of dark mirror baryons. In particular, dark disk scenarios are most likely to lead to the formation of mirror stars, which may provide an alternative discovery channel [66-69]. White dwarf cooling bounds are also most sensitive for disk-like mirror baryon distributions, probing $\epsilon$ as low as $10^{-12}-10^{-11}$ for a DM fraction of $10 \%$ [65].

The plasma-like nature of this DM component and its coupling to ordinary matter via the photon portal, which causes cross section enhancements at low momentum transfer, give rise to several complications. The most important of these is capture of mirror particles in the Earth. This can potentially affect the direct detection signal in two distinct ways. Firstly, a captured population of mirror nuclei could collisionally shield direct detection experiments from incoming mirror particles [144]. Secondly, in the case of the accumulation of a net mirror charge in the Earth, the resulting electrostatic repulsion could result in a suppression of the flux of incoming mirror particles that carry same charge, along with a reduction in their velocities. The sizes of these effects depends on the number of captured mirror particles of various species. This in turn depends, not just on the various capture processes, but also on the evaporation of captured mirror particles from the Earth and the Debye screening of the accumulated mirror charge by the ambient mirror plasma.

We perform a detailed study of the effects of mirror matter capture on direct detection in appendix $\mathrm{A}$, for kinetic mixings in the range of interest, $\epsilon \lesssim 10^{-9}$. We find that the captured mirror nuclei are primarily composed of $\hat{H}$, since the evaporation of $\hat{H}$ is much more efficient. Mirror electrons are ejected extremely efficiently from the Earth, and so the Earth eventually acquires a net positive mirror electric charge from the excess $\hat{H e}^{++}$. This net charge is efficiently screened by the ambient mirror plasma within a few $100 \mathrm{~km}$ of the Earth's surface. Our investigation is, to the best of our knowledge, the first such study to take the effects of this Debye screening into account. Our analysis shows that collisional shielding never plays a significant role in suppressing direct detection of mirror matter in our framework. The effects of electrostatic shielding are also negligible for $\epsilon \lesssim 10^{-11}$. For larger values of the kinetic mixing parameter, $\epsilon \gtrsim 10^{-10}$, electrostatic shielding can only modestly weaken the projected bounds on the kinetic mixing parameter $\epsilon$ by less than $50 \%$ (25\%) for mirror helium (hydrogen), while the mirror electron signal is unaffected or slightly enhanced. For $r_{\odot} \sim 0.01$, we find that future experiments probe values of $\epsilon$ much smaller than $10^{-10}$ and are therefore unaffected. Our sensitivity projections for $\epsilon \sqrt{r_{\odot}}$ from mirror nuclear recoils will therefore have at most a factor of 2 uncertainty, and only if $r_{\odot}$ is so small that 
the sensitivity boundary lies near or above $\epsilon \sim 10^{-10}$. The mirror-plasma effects of capture warrant further study, particularly in the context of more general dissipative DM models. However, since they do not affect our conclusions for MTH models, we neglect them in our analysis of direct detection below.

It is also possible for the self-interaction between two mirror particles in the solar system to result in one of the two particles becoming gravitationally bound in the Sun's gravitational well. However, for $r_{\odot} \lesssim 0.1$, the scattering length for two mirror baryons is so much larger than the size of the solar system that this effect is unlikely to be important. Finally, focusing of mirror DM in the gravitational wells of the Sun and Earth has the effect of increasing its local velocity at the Earth's surface. The low-velocity tails of distributions dominate capture, and so we take the speed gain when falling into the Earth's gravitational well into account in appendix A, but neglect all such effects in our direct detection calculations. Therefore, our sensitivity estimates below are somewhat conservative with regard to this effect.

\subsection{Direct detection via nuclear recoils}

We first consider the direct detection of mirror DM via nuclear recoils. We begin by reviewing the basic kinematics involved in the scattering of sub-nano-charged DM off nuclei. We then determine the reach of nuclear recoil experiments in the MTH framework.

\subsubsection{Review}

We begin by computing the cross section for a DM particle $X$ of mass $m_{X}$, mirror electric charge $Q_{X}$ and kinetic mixing parameter $\epsilon$ scattering off a single SM proton of mass $m_{p}$. Since the particles being scattered are nonrelativistic, the velocity of the incoming DM particle satisfies $v_{X} \ll 1$ in the lab frame. This then implies $E_{r} \ll m_{X}$, where $E_{r}$ is the recoil kinetic energy of the target particle after the collision. The matrix element for the $X p \rightarrow X p$ process, averaged over initial and summed over final particle spins is given by

$$
|\overline{\mathcal{M}}|^{2}=4 e^{4} \epsilon^{2} Q_{X}^{2} \frac{m_{X}^{2}}{E_{r}^{2}}
$$

(This formula is applicable to both electron and nuclear scattering.) The corresponding differential scattering cross section ${ }^{10}$ is given by,

$$
\frac{d \sigma_{p}}{d E_{r}}=\frac{2 \pi \alpha_{e m}^{2} \epsilon^{2} Q_{X}^{2}}{m_{p} v_{X}^{2} E_{r}^{2}} .
$$

This is to be contrasted with the case of WIMP DM, where nuclear scattering via a contact operator leads to a differential cross section that is independent of the recoil energy $E_{r}$. A typical collision with sub-nano-charged DM therefore produces much less recoil than a typical WIMP collision. The maximum nuclear recoil energy for a given DM velocity in the target rest frame is given by,

$$
E_{r}^{\max }=\frac{2 m_{p} m_{X}^{2} v_{X}^{2}}{\left(m_{p}+m_{X}\right)^{2}}
$$

\footnotetext{
${ }^{10}$ Note that the total integrated cross section $\int_{0}^{E_{r}^{\max }} d \sigma_{p} / d E_{r}$ is divergent, reflecting the infrared singularity expected in Rutherford scattering. In direct detection experiments this divergence is regulated by the minimum detectable recoil energy.
} 
Eqs. (4.6) and (4.7) can be applied to scattering off nuclei $N$ in the usual way, by replacing $m_{p}$ with $m_{N}$ and multiplying the l.h.s. of eq. (4.6) by $Z^{2} F^{2}\left(E_{r}\right)$, with $F$ being the Helm form factor [145]. The mass range of interest for mirror hydrogen and mirror helium DM in the MTH model is from about 1-5 GeV. Mirror electrons are too light to be detected via NR but can show up in ER experiments.

For completeness and ease of comparison to previously computed limits, we first consider the case of the standard collisionless single-component DM halo. For DM masses in the GeV range, the largest possible recoil energies are $\mathcal{O}(100 \mathrm{eV})$ on silicon, germanium or xenon targets. Most direct detection experiments sensitive to nuclear recoil search for WIMPs with masses above $\sim 10 \mathrm{GeV}$ and have energy thresholds in the keV range, severely limiting their sensitivity to sub-nano-charged DM. The next-generation SuperCDMS SNOLAB detectors [149] are the exception, with nuclear recoil energy thresholds as low as $40 \mathrm{eV} .{ }^{11}$ Using the information provided in [149] on minimum thresholds, signal efficiency and nuclear recoil spectra of backgrounds after imposing selection criteria, it is straightforward to compute exclusion limit projections for sub-nano-charged DM from the SuperCDMS SNOLAB Si/Ge HV/iZIP detectors. ${ }^{12}$ The Ge HV detector has the best sensitivity due to its low $40 \mathrm{eV}$ threshold, and we show that limit projection as the black curve in figure 4 . We stress, however, that the limits shown in figure 4 cannot be directly applied to the MTH model even in the case of an ionized halo, because they do not take into account the fact that the velocity distributions of the mirror particles are different from that of a standard collisionless CDM species.

\subsubsection{Nuclear recoils in the Mirror Twin Higgs}

It is straightforward to determine the limits on the MTH in the cases of ionized or atomic halos or disks, after taking into account their different velocity distributions as explained in section 4.1. The projected sensitivity of SuperCDMS HV Ge to mirror hydrogen and helium is shown in figure 5, for the ionized halo and ionized disk scenarios. For local $r_{\odot} \approx 0.01$, mixings as small as $\epsilon \sim 10^{-11}$ can be probed in the ionized halo case. Note that these experiments have greater sensitivity to mirror helium than to mirror hydrogen. For the ionized disk, mirror baryon recoil energies are two orders of magnitude lower than for the halo, since velocities go down by roughly a factor of ten. As a result, there is no NR signal. The momentum transfer for a NR collision with $\hat{\mathrm{H}}$ or $\hat{\mathrm{He}}$ is $q \approx \sqrt{2 m_{N} E_{r}} \sim \mathcal{O}(1-10 \mathrm{MeV})$, which is much larger than the energy scale corresponding to the size of a mirror atom $\hat{a}_{0}=\left(\alpha_{\mathrm{em}} m_{\hat{e}}\right)^{-1} \sim(\hat{v} / v \times 4 \mathrm{keV})^{-1}$. Therefore, the NR sensitivities for atomic disk and halo scenarios are very similar to the corresponding ionized cases.

\footnotetext{
${ }^{11}$ Both the HV and iZIP detectors are also sensitive to electron recoil, but present thresholds are too high to be useful for mirror DM detection. A possible exception are the fast mirror electrons in the ionized disk case, but even in that scenario the detectors have considerable background near the lower limit of their recoil sensitivity. We discuss dedicated semiconductor-based ER detectors in the next subsection.

${ }^{12}$ We derive these limits by assuming the given background distributions are accurate and optimizing, for each DM mass, the choice of a single $E_{r}$ interval which maximizes signal significance over the background. This gives a cross section limit that is a factor of a few better than the conservative "optimum interval method" used by [149], which does not make use of background subtraction. Our method is appropriate for a projection of the best possible reach.
} 


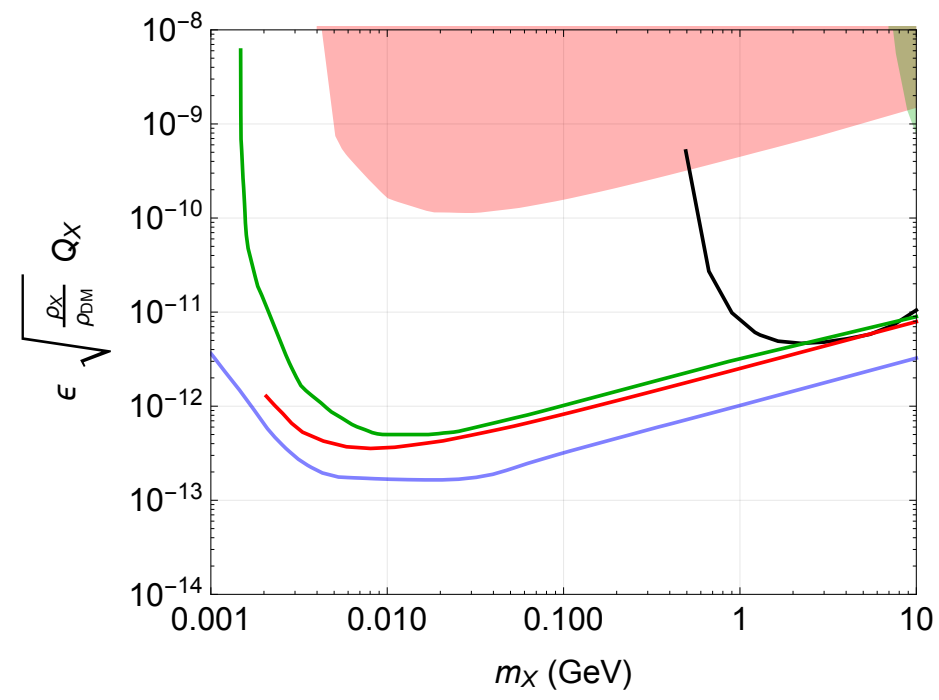

Existing ER limit,

Xenon 10, $\Delta \mathrm{E}_{e}>12.4 \mathrm{eV}$

$\square$ Existing NR limit,

LUX

- NR, SuperCDMS HV, Ge

$44 \mathrm{~kg} \cdot \mathrm{yr}, E_{\mathrm{nr}}>40 \mathrm{eV}$

— ER, SENSEI (Si),

$0.1 \mathrm{~kg} \cdot \mathrm{yr}, Q_{\mathrm{th}}=2 e^{-1}, \Delta \mathrm{E}_{e}>4.7 \mathrm{eV}$

- ER, Aluminium SC,

$1 \mathrm{~kg} \cdot \mathrm{yr}, \Delta \mathrm{E}_{e} \in(10 \mathrm{meV}, 10 \mathrm{eV})$

- ER, Graphene,

$1 \mathrm{~kg} \cdot \mathrm{yr}, \Delta \mathrm{E}_{e} \approx \mathrm{eV}$

Figure 4. Overview of current direct detection exclusion limits (shaded regions for Xenon10 and LUX) and the most relevant limit projections (lines) on sub-nano-charged DM, assuming the DM is distributed in a fully ionized, single-component standard halo. These limits do not directly apply to the MTH model due to the different velocity distribution and ionization of mirror baryons. $\rho_{X} / \rho_{\text {DM }}$ refers to the fraction of DM that is made up by the particular constituent $X$. Current Xenon10 limits from [146]. LUX [147] limits from [148]. We derived SuperCDMS NR limit projections using information from [149]. ER limit projections: SENSEI [150] limits from [148]. Superconducting aluminum target with $1 \mathrm{~kg} \cdot$ year exposure from [151], Graphene target with $1 \mathrm{~kg} \cdot y e a r$ exposure from [152].

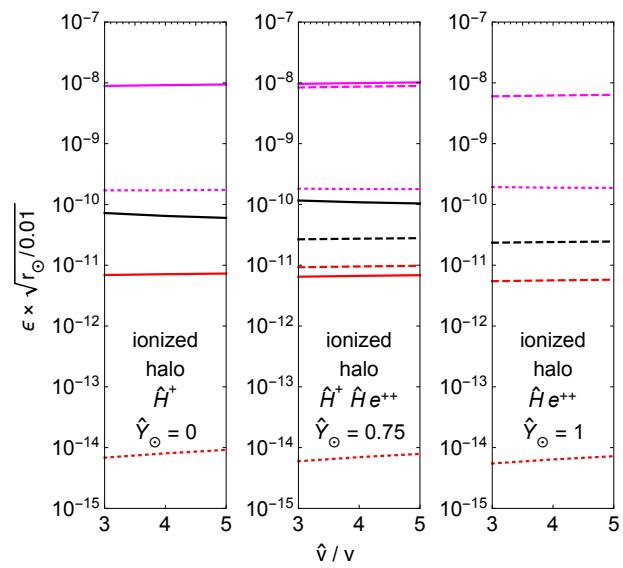

(a)
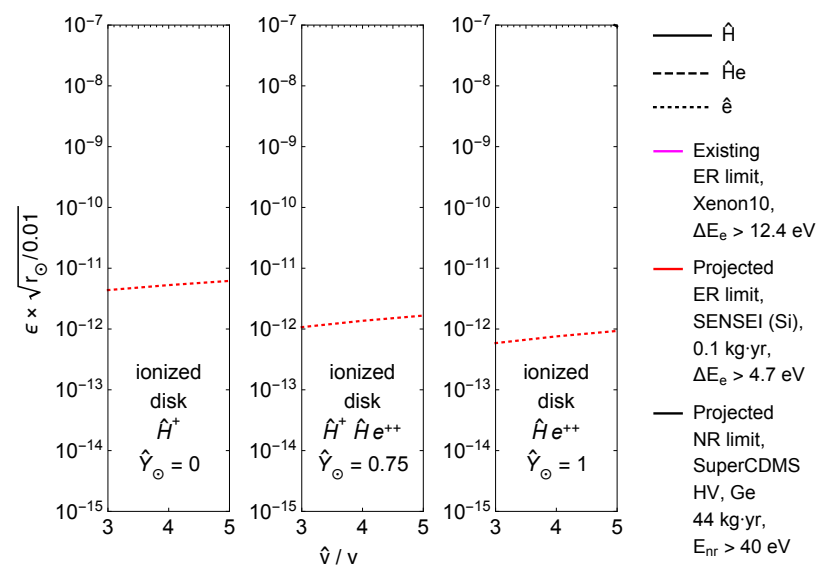

(b)

Figure 5. Constraints on photon kinetic mixing $\epsilon$ from direct detection of mirror $\mathrm{H}$, He and electrons at Xenon10 (ER, existing constraint) or SuperCDMS and SENSEI (NR and ER, projected constraints) for the ionized halo (a) and the ionized disk (b) distributions. The mirror particle masses and velocity distributions depend on $\hat{v} / v$ and the mirror helium fraction $\hat{Y}$, see section 4.1 for details. The limits for partial ionization are very similar to the shown cases of full ionization. Note that the disk limits do not take the increased local DM density into account. 


\subsection{Direct detection via electron recoils}

We now turn our attention to the direct detection of mirror DM via electron recoils. We first review the basic kinematics and existing detector technologies before computing the reach of these experiments in the MTH framework.

\subsubsection{Review}

DM direct detection via electron recoil has been studied in a variety of detector materials, including noble gases [146, 153, 154], semiconductors [148, 150, 153, 155, 156], scintillators [157], graphene [152], and superconductors [151, 158]. ER experiments have much lower energy thresholds than NR detectors and therefore have the potential to set very stringent limits on sub-nano-charged DM. Sensitivity projections for light vector mediators are typically expressed in terms of an effective interaction cross section $\bar{\sigma}_{e}$, which is related to the photon mixing parameter via the relation (see e.g. [148]),

$$
\bar{\sigma}_{e} \equiv \frac{\mu_{X e}^{2}}{16 \pi m_{X}^{2} m_{e}^{2}}|\overline{\mathcal{M}}|_{q^{2}=\alpha^{2} m_{e}^{2}}^{2}=\frac{16 \pi m_{X}^{2} Q_{X}^{2} \epsilon^{2}}{m_{e}^{2}\left(m_{e}+m_{X}\right)^{2} \alpha_{\mathrm{em}}^{2}},
$$

where we have made use of eq. (4.5). Here $m_{e}$ is the elctron mass while $\mu_{X e}$ represents the reduced mass of the DM-electron system. Existing ER limits from Xenon10, as well as projected sensitivities of future experiments that have been proposed, are shown in figure 4 . Note that these limits assume a standard single-component DM halo. To understand these limits, and how they are changed by the different mirror matter distributions in the MTH framework, some discussion of ER kinematics and detector technology is required.

The kinematics of DM-electron scattering is very different from DM-nuclear scattering, since for a standard halo, the electron is both the fastest and the lightest particle in the problem. Consequently, the typical momentum transfer in a collision is much smaller than in the case of DM-nucleus scattering, and so the fact that the electron forms a bound state in an atom or a bulk material must be taken into account. In particular, the electron does not have a definite momentum and very large momentum transfers $q$ are possible. Following the discussion in [148], the energy imparted to a bound electron can be obtained from energy conservation as

$$
\Delta E_{e}=\vec{q} \cdot \vec{v}_{X}-\frac{q^{2}}{2 \mu_{X N}},
$$

where $\mu_{X N}$ is the reduced mass of the DM and the nucleus. The likelihood of a given $q$ depends, in general, on a (possibly very complicated and material-dependent) form factor, but is typically of the order

$$
q_{\mathrm{typ}} \sim \mu_{X e} v_{\mathrm{rel}} \sim m_{e} v_{e} \sim \mathcal{O}(\mathrm{few}-10 \mathrm{keV})
$$

where we have assumed $m_{X} \gg m_{e}$. The relative velocity between the DM particle and the electron, $v_{\text {rel }}$, is dominated by the velocity of the bound state electron $v_{e} \sim Z_{\text {eff }} \alpha_{\text {em }} \sim$ $10^{-2} Z_{\text {eff }}$ in atoms (relevant for semiconductors, graphene and noble gases) or $v_{e} \sim v_{F} \sim 10^{-2}$ in Fermi-degenerate materials. (The effective charge $Z_{\text {eff }}$ is 1 for outer shell electrons and larger for inner shells.) This is much larger than the DM velocity $v_{X} \sim 10^{-3}$. 
For GeV-scale sub-nano-charged DM the second term in eq. (4.9) can be neglected, and the typical energy of a scattered electron depends linearly on the DM velocity. Assuming a standard halo profile with $v_{X} \sim 10^{-3}$, the typical energy imparted to the SM electron from a collision with mirror $\mathrm{H}$ or $\mathrm{He}$ is in the few $\mathrm{eV}$ range. For sub-nano-charged $\mathrm{DM}$ in a standard halo with mass closer to that of the electron, the second term in eq. (4.9) can no longer be neglected, and eq. (4.10) is no longer valid. Instead the typical electron recoil energy now scales quadratically with DM velocity, $\Delta E_{e}^{\max }=\frac{1}{2} \mu_{X N} v_{X}^{2}$. Then the typical energy imparted to a SM electron from a collision with a DM particle with the mass of a mirror electron in a standard halo is only of order $0.1 \mathrm{eV}$.

With this parametric understanding of ER kinematics we now consider the various experiments in turn and discuss their differences.

- Ionization in noble gases $[146,153,154]$ (shaded red region in figure 4). In detectors based on noble gases such as xenon, NR from DM collisions is detected via prompt scintillation as the excited atom returns to its ground state ("S1" signal) as well as ionization, where the liberated electrons are accelerated by a strong external electric field, escape the liquid phase of the detector and release scintillation light as they traverse the gaseous phase ("S2" signal). These detectors can also be sensitive to electron recoil if the $\mathrm{S} 1$ signal requirement is dropped. The best current limits on sub-nano-charged DM for masses below $10 \mathrm{GeV}$ were derived in [154] (refined in [146]) using an S2-only Xenon10 dataset with a single-electron ionization threshold [159]. This allows ER events to be detected as long as the collision imparts at least $12.4 \mathrm{eV}$ of energy to the electron and liberates it from the outer shell. Higher energy recoils can be distinguished by higher levels of ionization. The relatively high levels of detector specific background limit sensitivity. Current limits were obtained without a background model, assuming all the observed events to arise from DM scattering. ${ }^{13}$

- Ionization in semiconductors $[148,153,155,156]$ (red curve in figure 4). In silicon (germanium), the minimum electron energy required to eject an electron is $1.11 \mathrm{eV}$ $(0.67 \mathrm{eV})$. As in noble gas detectors, the resulting ionization(s) are picked up by accelerating the liberated electrons in an external electric field. Detecting a single ionization is very challenging, but the SENSEI collaboration [150, 162] was recently funded to build a 100-gram silicon detector capable of detecting ER, with an ionization threshold that could be as low as $Q_{\text {th }}=2$, corresponding to $\Delta E_{e}>4.7 \mathrm{eV}$. The resulting sensitivity [148] is shown as the red curve in figure 4 for the standard single-component halo. Note that the sensitivity extends down to DM masses in the mirror electron range, relying on the tail of the mirror DM velocity distribution to achieve an electron recoil above threshold.

\footnotetext{
${ }^{13}$ While this paper was in preparation, LUX [160] and Xenon1T [161] published analyses based on searches for electron recoils in mirror models. The LUX analysis relied on assumptions about collisional shielding that do not apply in our case (see appendix A). Nevertheless, it may have better sensitivity than Xenon10 to the halo case. The Xenon1T analysis improves the reach in $\epsilon$ by one order of magnitude for GeV DM masses compared to Xenon10, but the projected reach of SuperCDMS via nuclear recoil that we compute is still more sensitive. For mirror electrons in the MeV range, Xenon10 is more sensitive than Xenon1T.
} 
- Scintillators [157]. An alternative path to low-threshold ER detection makes use of scintillators. Here the experimental observable is the scintillation light emitted as the excited atoms relax to their ground state. While this has slightly worse sensitivity than ionization in semiconductors due to the $\sim 6 \mathrm{eV}$ thresholds of the readily available scintillation materials such as NaI, it may allow for lower backgrounds since no electric field is required to manipulate the liberated electron.

- Graphene [152] (green curve in figure 4). Graphene is a very attractive target for ER DM direct detection. It has an energy threshold $\Delta E_{e} \gtrsim \mathrm{eV}$ comparable to semiconductors, and the momentum of the ejected electron can be directly determined without relying on secondary excitations, allowing for directional DM detection as well as a very precise measurement of the ER spectrum. Furthermore, this proposal could be realized in the near future by running the PTOLEMY experiment [163] with bare rather than tritium-holding graphene surfaces. Achievable sensitivity, calculated by [152] assuming backgrounds can be rejected, is shown as the green curve in figure 4 .

- Superconductors $[151,158]$ (blue curve in figure 4). A DM collision with electrons in a superconductor could disrupt a Cooper pair and create two propagating quasiparticle excitations above the Fermi sea. The band gap for this transition is tiny, of order $10^{-3} \mathrm{eV}$, allowing in principle for DM detection with extremely low thresholds. Once these excitations are produced in a large volume superconducting substrate, they must be concentrated and collected in a small volume absorber, and read out with sensors like Transition Edge Sensors (TES) or Microwave Kinetic Inductance Devices (MKID). One promising approach discussed in [151], (see also [164]), is the use of a single aluminum crystal in the superconducting state, allowing for efficient propagation and collection of the produced excitations. Estimated reach for sub-nano-charged DM with $1 \mathrm{~kg}$ year of exposure is shown as the blue curve in figure 4 for a readout sensor dynamic range of $10 \mathrm{meV}-10 \mathrm{eV}$. The solar neutrino background has been included in this sensitivity estimate, which scales linearly with ER energy in almost all of the relevant energy range. The time scale for implementing superconductors as ER DM detectors is probably longer than for the other technologies discussed here. The required $\mathcal{O}(1 \mathrm{meV})$ sensitivities have not yet been achieved, though they are theoretically possible in TES and MKID sensors, and various engineering approaches for improving sensitivity have been proposed.

Recent ideas like polar target materials $[165,166]$ could play an important role similar to superconductors due to their very low thresholds.

Figure 4 makes it clear that superconductors, graphene and semiconductors have comparable sensitivities to sub-nano-charged DM in a standard single-component halo. We now discuss the role each of these detection technologies could play in the detection of mirror baryons and electrons. 


\subsubsection{Electron recoils in the Mirror Twin Higgs}

We now compute the sensitivities of ER experiments to mirror baryons and electrons for the different benchmark distributions defined in section 4.1. For our quantitative analysis we focus on Xenon10, on which the best current constraints are based, and SENSEI (ionization in silicon), which serves as an example of the sensitivity achievable in the near future. We also give a qualitative discussion of the role that other technologies, such as graphene and superconductors, can play.

For the Xenon10 ER constraint, we compute the limits in the same manner as in ref. [146], but taking into account the different velocity distributions of the individual mirror electron and baryon components. ${ }^{14}$ As is clear from the magenta curves in figure 5 , Xenon10 already constrains the nano-charged regime in the ionized halo scenario. The signals from mirror hydrogen and helium in this case are not very different from those of a standard halo, leading to a bound of $\epsilon \lesssim 10^{-8}$ for $r_{\odot} \sim 0.01$, as expected from figure 4 . For mirror electrons, the situation is quite different. They would not be detectable at Xenon10 if they exhibited a standard halo velocity distribution, due to the high single ionization threshold of $12.4 \mathrm{eV}$ compared to the typical recoil energy. However, in the ionized halo, their increased speed allows them to easily liberate electrons from the outer shells of xenon. It is worth noting that the kinematics of this collision are quite different from the discussion following eq. (4.10). Since the mirror electron is now the fastest particle in the problem, we might naively expect $q_{\mathrm{typ}} \sim m_{e} v_{\hat{e}}$. However, the atomic form factor of xenon, as well as the $1 / q^{4}$ suppression in the scattering cross section, still favor momentum transfers at or below $\alpha m_{e}$. Instead, the increased mirror electron speed allows sizable $\Delta E_{e}$ to be generated from collisions with very low momentum transfer, at or much below $\alpha m_{e}$. The same $1 / q^{4}$ cross section dependence then leads to a huge rate enhancement for fast mirror electrons, allowing Xenon10 to set limits on $\epsilon$ of order $10^{-10}$ for the ionized halo with $r_{\odot} \sim 0.01$.

Xenon10 does not set limits on the ionized disk scenario; mirror electrons now have a speed comparable to that expected from a standard halo, and as shown in figure 4, this is not sufficient to ionize xenon. Mirror baryons are slower by an order of magnitude, leading to a corresponding decrease in recoil energy, which is also insufficient to ionize xenon.

We repeat this calculation for the ionization of silicon in the SENSEI experiment [150], using the public QEDark code made available by the authors of [148] to compute the signal rate, which includes the fully pre-computed crystal form factor. The corresponding background-free ER limit projections (4 expected events) are shown as red lines in figure 5. The discussion of kinematics and rate enhancement for fast mirror electrons is very similar to the case of xenon. An important difference is that, due to the lower ionization threshold in silicon, the signal from fast mirror electrons in the ionized halo is even more enhanced than in xenon, making it possible to see them even in the ionized disk scenario.

Mirror baryons can be discovered in SENSEI for $\epsilon \gtrsim 10^{-11}$ in the ionized halo with $r_{\odot} \sim 0.01$, while mirror electrons give rise to a detectable signal even for tiny $\epsilon \sim 10^{-14}$, a truly remarkable sensitivity that can probe mixings even smaller than the expected

\footnotetext{
${ }^{14}$ We are very grateful to Tien-Tien Yu for supplying us with the necessary code, which includes the atomic form factors for xenon.
} 
gravity-mediated contributions [64]. In the ionized disk scenario, mirror electrons are detectable for $\epsilon \gtrsim 10^{-12}$. This will allow a very effective probe of the sub-nano-charged regime. Mirror baryons in the ionized disk are very challenging to detect due to their low recoil, both in NR and ER experiments. This represents a great opportunity for a future superconductor or polar material based ER detector, which would be able to probe this scenario very effectively due to its tiny $\mathrm{meV}$ thresholds.

Future graphene-based detectors [152] are likely to have a sensitivity comparable to SENSEI for the same exposure due to their similar ionization energies. However, graphene has the unique ability to detect the direction of the DM impact, which could provide another useful handle for diagnosing the mirror baryon distribution. For example, in the ionized disk scenario as described in section 4.1, we do not expect significant annual modulation in the strength of the signal, but to the small extent that directional bias exists in the impact of mirror electrons, events would be sensitive to the direction the Earth is currently heading around its orbit. This would constitute a striking signal of a mirror baryonic disk.

What if the mirror particles form atoms either in a disk or a halo? Since the typical momentum transfer in the collision of a mirror atom with a nucleus is much larger than the binding energy of a mirror atom, the NR signal is expected to be very similar to that in the ionized case. However, the situation with regard to ER signals is very different. As discussed above, the typical momentum transfer in collisions between mirror baryons and a visible bound electron is $q_{\mathrm{typ}} \sim$ few $-10 \mathrm{keV}$, see eq. (4.10). The characteristic size of mirror atoms is given by the mirror Bohr radius, $\hat{a}_{0}=\left(\alpha_{\mathrm{em}} m_{\hat{e}}\right)^{-1} \sim(\hat{v} / v \times 4 \mathrm{keV})^{-1}$. Since the momentum transfer corresponds to length scales similar to or larger than a mirror atom, the total scattering rate will be suppressed by mirror atomic form factors. (The velocity distributions would also change slightly due to the absence of free mirror electrons but, just as for NR, this is a less important effect.) For $q_{\text {typ }} \ll \hat{a}_{0}^{-1}$, this cross section suppression is $\sim \hat{a}_{0}^{4} q_{\text {typ }}^{4}$ [85]. Compared to the ionized case, the corresponding reduction in sensitivity to $\epsilon$ is roughly

$$
\hat{a}_{0}^{2} q_{\mathrm{typ}}^{2} \sim\left(\frac{v}{\hat{v}}\right)^{2}\left(\frac{v_{e}}{\alpha_{\mathrm{em}}}\right)^{2} .
$$

The first term is $\sim \mathcal{O}(0.1)$ for our parameters of interest, while $v_{e} / \alpha_{\mathrm{em}}$ is $\sim \mathcal{O}(1)$ for outer ionization electrons and in superconductors. Therefore, the $\epsilon$ sensitivities for the atomic disk/halo are at most an order of magnitude or so weaker than the corresponding sensitivity for the ionized disk/halo. Compared to the ionized case, the ER spectrum will also be modified. Since the scattering proceeds via the dipole moment of the mirror atom, there is no separate mirror electron signal.

The atomic halo would therefore be clearly discoverable via NR and ER detection of mirror baryons, though with reduced ER sensitivity. Both the different signal rate and recoil spectrum shape can be used to distinguish this scenario from the ionized halo or disk. The atomic disk is an extremely challenging case. The absence of free and fast mirror electrons means that only a future superconductor or polar material based detector with very low threshold has a chance to detect mirror atoms. Even then the rate would be suppressed by DM atomic form factors. That being said, the absence of events in all other detectors would make a discovery at such a low-threshold detector a striking signal of an atomic disk. 


\begin{tabular}{|l|l|l|l|l|}
\hline & ionized halo & ionized disk & atomic halo & atomic disk \\
\hline ER $\hat{H}, \hat{H e}$ & $\epsilon \sim 10^{-8}$ & no signal & AFF: $\epsilon \sim 10^{-7}$ & no signal \\
\hline ER $\hat{e}$ & $\epsilon \sim 10^{-10}$ & no signal & no signal & no signal \\
\hline
\end{tabular}

Table 3. Summary of existing constraints on dark photon mixing $\epsilon$ from direct detection of mirror baryons and electrons via electron recoil at Xenon10. Here we assume $r_{\odot} \sim 1 \%$ for all cases. Limits scale with $r_{\odot}^{-1 / 2}$. Note that for a given cosmic mirror baryon abundance $r_{\text {all }}$, the local density $r_{\odot}$ is likely higher in the disk cases compared to the halo. In the left three columns, the reason for the sensitivity reduction compared to the ionized halo is given. AFF $=$ Atomic Form Factor, RR $=$ Reduced Recoil, see text for details.

\begin{tabular}{|l|l|l|l|l|}
\hline & ionized halo & ionized disk & atomic halo & atomic disk \\
\hline NR $\hat{H}, \hat{H e}$ & $\epsilon \sim 10^{-11}$ & RR: no signal & $\epsilon \sim 10^{-11}$ & RR: no signal \\
\hline ER ĤH $\hat{H e}$ & $\epsilon \sim 10^{-11}$ & RR: SC only? & AFF: $\epsilon \sim 10^{-10}$ & RR and AFF: SC only? \\
\hline ER $\hat{e}$ & $\epsilon \sim 10^{-14}$ & $\epsilon \sim 10^{-12}$ & no signal & no signal \\
\hline
\end{tabular}

Table 4. Summary of projected sensitivities to dark photon mixing $\epsilon$ from direct detection of mirror baryons and electrons via nuclear recoil at SuperCDMS HV Ge, and via electron recoil at SENSEI (or a hypothetical superconductor detector). Here we assume $r_{\odot} \sim 1 \%$ for all cases. Limits scale with $r_{\odot}^{-1 / 2}$. Note that for a given cosmic mirror baryon abundance $r_{\text {all }}$, the local density $r_{\odot}$ is likely higher in the disk cases compared to the halo. In the left three columns, the reason for the sensitivity reduction compared to the ionized halo is given. AFF $=$ Atomic Form Factor, RR = Reduced Recoil (meaning detection may require a superconductor or polar material based detector), see text for details.

\subsection{Characterization of the dark sector}

In tables 3 and 4 we summarize the present limits and projected future sensitivities of NR and ER direct detection experiments to mirror dark matter. In the case of an ionized halo, the existing Xenon10 constraints on ER already probe some of the nano-charged regime, but the other scenarios are presently unconstrained. With future experiments, the ionized halo, ionized disk and atomic halo distributions can all be effectively probed, with clearly distinct patterns of detection. This may allow these different distributions to be distinguished. The atomic disk scenario is however very challenging, and will rely on the future development of superconductor-based ER detectors with extremely low thresholds. ${ }^{15}$ Once that capability exists, it can also be discovered, and distinguished from the other mirror matter distributions. Directional detection in graphene-based ER detectors, as well as the characteristic annual modulation of any detected signal at different detectors, would provide additional information that could help resolve any remaining degeneracy between different distributions of the sub-nano-charged DM component.

We close this section with the argument that a detailed study of the distribution of recoil energies in signal events can be used to further characterize the dark sector. In particular,

\footnotetext{
${ }^{15}$ Direct searches of mirror stars [66-69], which are more likely to form if the mirror baryons have collapsed into a cold disk, may provide a more immediate probe of this scenario.
} 


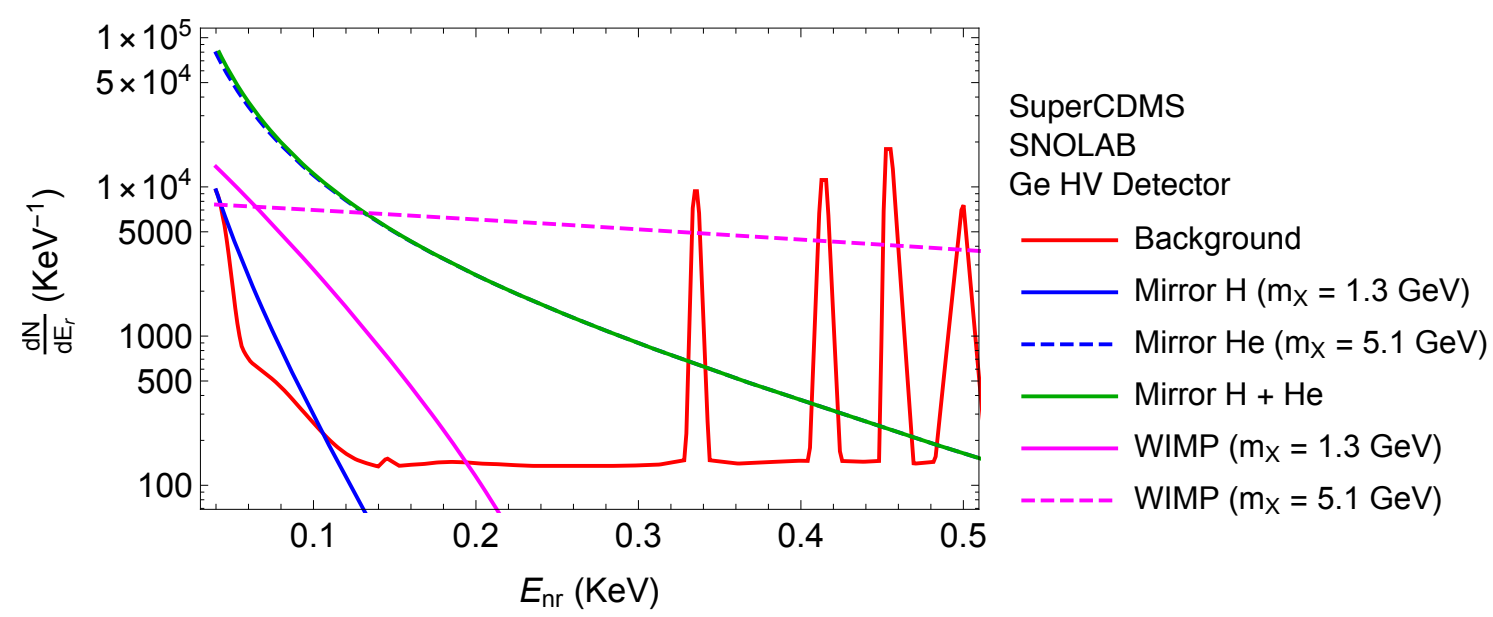

Figure 6. Recoil spectrum in the SuperCDMS SNOLAB Ge HV detector assuming ionized halo $\mathrm{DM}$ distribution. For mirror $\mathrm{H}$ and $\mathrm{He}$, assume local values $r_{\odot}=0.01, \hat{Y}_{\odot}=0.75, \hat{v} / v=4$, and $\epsilon=3 \times 10^{-10}$. We compare to a WIMP with $m_{X}=1.3(5.1) \mathrm{GeV}$ and $\sigma_{n X}=0.6(1.1) \times 10^{-42} \mathrm{~cm}^{-2}$.

once the nature of the mirror baryon distribution has been determined by correlating data from different detectors as shown in table 4, the detailed recoil energy spectra can potentially be used to establish that DM is multi-component, and also to distinguish the signal from that of a primary WIMP DM component. As a demonstration we consider mirror baryons distributed in an ionized halo giving rise to a signal in a NR detector, such as SuperCDMS SNOLAB Ge HV. Figure 6 shows the nuclear recoil spectra for $\hat{\mathrm{H}}$ and $\hat{H} e$ for $\epsilon=3 \times 10^{-10}$, as well as WIMP signals of comparable statistical significance and identical masses. This corresponds to of order a thousand signal events from mirror baryons. The recoil spectra from $\hat{\mathrm{H}}$ and $\hat{\mathrm{H} e}$ are clearly very different. Even though the mirror hydrogen signal is much smaller than the mirror helium signal, their combined recoil spectrum can be distinguished from either individual component. This can be used to establish that the mirror DM consists of more than one type of nucleus, and allows for direct measurement of the local mirror helium fraction $\hat{Y}_{\odot}$. Furthermore, by establishing that the masses and charges of mirror hydrogen and helium are integer multiples of each other, these experiments may be able to distinguish the mirror nature of the theory.

For comparison, we show the distribution of recoil energies that would be expected from WIMPs of the same masses ( 1.3 or $5.1 \mathrm{GeV}$ in this case). It is clear that the WIMP and mirror baryon signals can also be distinguished, since the distribution of WIMP events goes out to much larger $E_{r}$ than the sub-nano-charged DM component. We have further verified that given a few hundred signal events, the nuclear recoil spectra of mirror hydrogen and mirror helium can be reliably distinguished from that of a WIMP without any prior assumptions about the WIMP mass.

Combining and correlating data from different detectors can reveal additional information that could help distinguish mirror DM from WIMPs. For example, in an ionized halo the fast mirror electron signal in ER detectors would stand out because of its high recoil energies. By contrast, a dominant WIMP-like DM component may not even produce a 
signal at ER experiments. Shape analysis of the spectrum of signal events could also reveal, for example, whether the sub-nano-charged DM is atomic or ionized.

This study demonstrates the extraordinary power of direct detection experiments in discovering and probing a rich dark sector. In the future, a determination of the distribution, ionization, and multi-component nature of sub-nano-charged matter at direct detection experiments could provide a multi-pronged verification of the mirror nature of the MTH model.

\section{Conclusions}

The MTH framework connects the solution of the Higgs hierarchy problem to striking cosmological signatures from the early universe. A crucial aspect of this scenario is the likely existence of an asymmetric mirror matter component that constitutes a subdominant but dynamically rich fraction of DM. In this work we have studied the behavior of these relic mirror particles during galaxy formation, and their resulting unique multi-component signatures in DM direct detection searches. Apart from being the first such detailed study of mirror baryons, several of the effects we analyze in detail for the first time, such as dark plasma screening due to DM capture in the Earth, can be important for more general models of atomic dark matter. Our analysis lays the groundwork for future studies that will greatly enhance our understanding of this broad class of theories.

In contrast to conventional mirror matter models, the requirement of solving the Higgs hierarchy problem and satisfying cosmological constraints places limits on the mass and temperature of the mirror particles. Although detailed $N$-body simulations incorporating magnetohydrodynamic effects would be necessary to obtain a precise time evolution of the mirror plasma distribution, we can still use the known properties of twin particles to estimate their cooling rates and obtain a qualitative understanding the current distribution of this DM component. We find that MTH baryons forming a sub-percent DM fraction $r_{\text {all }} \lesssim 0.01$ are unlikely to have had enough time since the formation of the Milky Way to collapse into a dark disk. For greater DM fractions, the mirror matter distribution today is right near the threshold of being either halo- or disk-like. We therefore consider the possibility that local mirror baryons are either fully ionized or fully atomic due to unknown mirror astrophysics. These distinct possibilities for twin profiles today generate very distinct signals in different types of DM detectors, assuming the twin photon mixes with the SM photon at the levels expected from gravitational effects [64]. Measurements at various experiments can then establish a unique fingerprint of the twin sector, allowing us to probe its multi-component nature, ionization, local distribution, and also MTH model parameters such as $\hat{v} / v$. Our analysis shows that the relic MTH mirror particles in the universe can give rise to distinctive signatures that are sensitive to the detailed properties of the twin sector. Our analysis is by design robust with respect to the large astrophysical uncertainties, demonstrating the presence of distinctive direct detection signals for atomic or ionized dark halos or disks. In fact, any observed signal can help understand the unknown mirror astrophysics. 
Direct detection is especially sensitive in scenarios in which the mirror matter remains hot and distributed in the form of a halo down to the present day. This makes direct detection complementary to signals from white dwarf cooling [65] and mirror stars [66-69]. These astrophysical searches for mirror baryons are particularly sensitive in dark disk scenarios, since this leads to more accumulation of dark matter in SM stars and favors the formation of mirror stars. In this case, direct detection searches are more difficult but still have significant sensitivity.

Clearly, the combination of direct detection experiments and astrophysical searches greatly enhances our chances of discovering or excluding the asymmetrically reheated MTH and other mirror matter scenarios. If any or several of these signals were observed and correlated with the expected Higgs decay signal $\operatorname{Br}(h \rightarrow$ invisible $) \sim(v / \hat{v})^{2}$ at the LHC or a future collider, the night sky would illuminate a picture of naturalness that establishes the existence of the twin universe.

Note added. While this work was being completed, the Xenon1T experiment reported an excess of a few-keV electronic recoil events [167], which admits a variety of DM interpretations. It is interesting to note that mirror electrons within the MTH model might be able to account for such an excess [168], due to their higher velocity in the mirror plasma relative to mirror nuclei. However, we defer a careful study of this possibility for future work.

\section{Acknowledgments}

We thank Asimina Arvanitaki, Masha Baryakhtar, John Dubinski, Daniel Egana-Ugrinovic, Rouven Essig, Akshay Ghalsasi, Junwu Huang, Rabindra Mohapatra, Norman Murray, Shmuel Nussinov, Jessie Shelton, Thomas Quinn, Tien-Tien Yu, Yiming Zhong, and Yue Zhao for helpful discussions. We also thank Ina Flood for helpful corrections to the appendix. ZC is supported in part by the US National Science Foundation under Grant Number PHY-1914731. The research of DC is supported by a Discovery Grant from the Natural Sciences and Engineering Research Council of Canada, and by the Canada Research Chair program. In the early stages of this study, the work of DC and MG was also supported by the by the Maryland Center for Fundamental Physics. MG is supported in part by the Israel Science Foundation (Grant No. 1302/19). The work of YT was supported in part by the National Science Foundation under grant PHY-1914731 and by the Maryland Center for Fundamental Physics. YT was also supported in part by the National Science Foundation under grant PHY-2014165. ZC and MG are also supported in part by the US-Israeli BSF grant 2018236. YT thanks the Aspen Center for Physics, which is supported by National Science Foundation grant PHY-1607611. 


\section{A Capture of mirror matter in the Earth}

In this appendix we consider the accumulation of mirror matter in the Earth and its effects on direct detection in the scenario discussed in this paper. Our analysis shows that for $\epsilon \lesssim 10^{-9}$, the accumulation of mirror matter does not have a significant impact on the direct detection prospects of either mirror nuclei or mirror electrons. The assumptions we make in this analysis are chosen such as to overestimate the rate of capture of mirror matter, and consequently its effects on direct detection. Our results are therefore somewhat conservative.

In what follows we study the accumulation of mirror matter for $\epsilon \lesssim 10^{-9}, \hat{v} / v \in(3,5)$ and local mirror helium fractions $\hat{Y}_{\odot} \in(0,1)$, considering both the disk and halo distributions as outlined in section 4.1. The equilibrium accumulated mirror particle number densities are determined by the rates of capture and evaporation, which in turn depend on the accumulated mirror electric charge of the Earth and the resulting screening by the ambient mirror plasma. In our analysis we assume that all mirror matter is fully ionized. This assumption is conservative, since capture is suppressed for mirror atoms.

Mirror nuclei are captured by scattering off SM nuclei in the Earth. Mirror hydrogen evaporates fairly efficiently but mirror helium accumulates, resulting in the Earth acquiring a net positive mirror electric charge. The resulting repulsive force arrests further capture of mirror baryons and gives rise to an equilibrium population of captured mirror helium nuclei. Mirror electrons are mainly captured by inelastic scattering with bound atomic SM electrons in the Earth. After being captured, mirror electrons are very efficiently evaporated by scattering off conduction band electrons in the earth's metallic core. This results in an equilibrium number of captured mirror electrons that is fairly small and quite insensitive to the number density of captured mirror helium unless the net positive charge of the Earth from the captured mirror nuclei is very large. The total number of accumulated mirror particles is always small enough that the capture process is dominated by interactions with SM matter in the Earth, rather than interactions with mirror particles that have already been captured.

The positive mirror electric charge arising from the captured mirror nuclei is screened by the ambient mirror plasma. The characteristic length scale for this screening is at most of order $\sim 10^{-1} R_{\text {Earth }} \sim 500 \mathrm{~km}$, and mirror particles that are further away from the Earth's surface than this do not experience a large electric field. Nevertheless, for $\epsilon \gtrsim 10^{-10}$ it is very important to take this mirror electric screening effect into account, since it greatly modifies the mirror electric potential in the neighborhood of the Earth and thereby affects the equilibrium population of captured mirror nuclei.

A captured population of mirror nuclei could impact direct detection in two distinct ways:

1. Collisional shielding: collisions of incoming mirror particles with the population of accumulated mirror baryons could act as a shield, preventing the incoming mirror particles from reaching direct detection experiments. This collisional shielding effect was taken into account by the recent LUX analysis for $\mathbb{Z}_{2}$-symmetric mirror DM [160], based on the analysis in [144]. However, in the framework we are considering, we find that this effect is negligible for $\epsilon \lesssim 10^{-9}$. 
2. Electrostatic shielding: one might expect that the accumulated positive mirror charge due to captured He would electrostatically repulse incoming mirror nuclei, suppressing their direct detection signal to negligible levels. However, we show that electrostatic effects only matter for $\epsilon \gtrsim 10^{-10}$ and also act to suppress the population of captured mirror particles, therefore resulting in only a modest $\mathcal{O}(1)$ reduction in the signal rate at direct detection experiments. Since the proposed experiments we consider probe far smaller kinetic mixings than $10^{-10}$ for $r_{\odot} \sim 0.01$, this effect only introduces a $\lesssim 50 \%$ uncertainty in the projected reach for $\epsilon \sqrt{r_{\odot}}$ if $r_{\odot} \ll 0.01$.

The projections for mirror baryon direct detection that we present in this paper are therefore at most modestly affected by capture inside the Earth. While these effects are interesting and deserve future study, particularly in the context of more general dissipative DM models, we are justified in neglecting them in our analysis of direct detection in the MTH framework in section 4.

We now proceed to discuss the capture of mirror matter, mirror plasma screening, and mirror matter evaporation in detail. Throughout, we denote the free, or ambient, mirror particle densities in the local mirror plasma far away from the Earth by $n_{i}^{F}$ (completely determined in terms of $r_{\odot}$ and $\hat{Y}_{\odot}$ for $i=\hat{\mathrm{e}}, \hat{\mathrm{H}}, \hat{\mathrm{He}}$ ). Number densities of mirror particles captured in the Earth are denoted $n_{i}^{C}$. We focus our discussion on the limit where the mirror baryons are entirely composed of twin helium, $\hat{Y}_{\odot}=1$, since their evaporation is less effective than for twin hydrogen, and consequently their effect on direct detection is larger. Other values of $\hat{Y}_{\odot}$ do not qualitatively affect our conclusions. The MaxwellBoltzmann distributions of mirror helium and mirror electrons are determined by their velocity dispersions, as discussed in section 4.1 . For the disk (halo) case, $v_{\odot \hat{H} e} \sim 11 \mathrm{~km} / \mathrm{s}$ $(120 \mathrm{~km} / \mathrm{s})$ and $v_{\odot \hat{e}} \sim 500-700 \mathrm{~km} / \mathrm{s}(5000-7000 \mathrm{~km} / \mathrm{s})$ for $\hat{v} / v \sim 3-5$. We neglect the speed of the Earth relative to the mirror plasma in this discussion, since it is not expected to qualitatively alter our conclusions. In all cases, our baseline assumption is that mirror baryons make up $5 \%$ of the local DM density, i.e. $r_{\odot}=0.05$, but we discuss how our results can be extended to more general $r_{\odot}$ values as well.

\section{A.1 $\epsilon \lesssim 10^{-11}$}

We first estimate the effects of capture on direct detection for relatively low values of the kinetic mixing, $\epsilon \lesssim 10^{-11}$, for which the accumulated mirror charge in the Earth is small compared to the ambient density of the mirror plasma.

\section{A.1.1 Capture of mirror helium}

We begin by considering the capture of mirror helium. Depending on the velocity of the incoming mirror particles, capture may primarily arise either through multiple soft scatterings with the material in the Earth, or through a single hard scattering process.

For an incoming nano-charged particle $X$, the rate of kinetic energy loss per unit distance traveled inside the Earth due to multiple soft scatterings is given by,

$$
\frac{d E_{k}}{d r}=-n_{T} \int \frac{d \sigma}{d E_{R}} E_{R} d E_{R}=-n_{T} \int \frac{m_{X} \pi \alpha^{2} \epsilon^{2} Q_{X}^{2} Z^{2}}{m_{T} E_{k} E_{R}} d E_{R}=-n_{T} \frac{m_{X} \pi \alpha^{2} \epsilon^{2} Q_{X}^{2} Z^{2}}{m_{T} E_{k}} \log \frac{E_{R}^{\max }}{E_{R}^{\min }} .
$$


Here we assume that the energy loss mainly arises from Rutherford scattering with nuclei. The parameters $n_{T}, m_{T}$, and $Z$ correspond to the number density, mass, and charge of the atoms in the Earth representing the scattering target. The generalization to a more realistic material composition is straightforward. The parameter $E_{R}$ represents the recoil energy and $E_{R}^{\max }$ its maximum value, given by

$$
E_{R}^{\max }=\frac{4 m_{T} m_{X} E_{k}}{\left(m_{T}+m_{X}\right)^{2}} .
$$

The parameter $E_{R}^{\min }$ denotes the infrared scale at which the finite size of the atom cuts off the interaction, given roughly by $E_{R}^{\min } \sim\left(m_{e} \alpha\right)^{2} / m_{T}$. Assuming a constant density and composition of the Earth and neglecting the energy dependence of the logarithm, the energy loss with distance travelled is given by

$$
E_{k}(r)=E_{k}(0) \sqrt{\frac{r_{E}^{\text {Rut }}-r}{r_{E}^{\text {Rut }}}},
$$

where $r_{E}^{\text {Rut }}$ corresponds to the penetration depth needed to lose all of the initial energy,

$$
\begin{aligned}
r_{E}^{\text {Rut }} & \sim \frac{1}{4} m_{X} v_{X}^{2}\left(\frac{d E_{k}}{d r}\right)^{-1} \sim m_{X} m_{T} v_{X}^{4}\left(8 \pi \alpha^{2} \epsilon^{2} Q_{X}^{2} Z^{2} n_{T} \log \frac{E_{R}^{\max }}{E_{R}^{\min }}\right)^{-1} \\
& \sim R_{\text {Earth }}\left(\frac{v_{X}}{20 \mathrm{~km} / \mathrm{s}}\right)^{4}\left(\frac{2 \cdot 10^{23} \mathrm{~cm}^{-3}}{n_{T}}\right) \frac{m_{T}}{16 \mathrm{GeV}} \frac{m_{X}}{5 \mathrm{GeV}}\left(\frac{8}{Q_{T}}\right)^{2}\left(\frac{2}{Q_{X}}\right)^{2}\left(\frac{10^{-10}}{\epsilon}\right)^{2}
\end{aligned}
$$

Here $v_{X}$ is the mirror particle velocity when it enters the Earth, and we use the representative values of the charge $Q_{X}=2$ and mass $m_{X} \sim 5 \mathrm{GeV}$ of mirror helium as an example. The most effective target inside the Earth is oxygen, since it is fairly abundant and light. We have checked that a more careful integration of eq. (A.1) yields a comparable result.

We regard a mirror nucleus as captured if it enters the Earth and loses enough energy such that it either gets stuck inside the Earth, or emerges from the Earth with velocity less than the escape velocity, $v_{\text {esc }, i}$. As we shall see, the Earth eventually acquires a net positive mirror electric charge from the capture of $\hat{H e}$, and the escape velocities of mirror particles depend on the net charge. In the absence of any net captured charge the escape velocity is the same for all species and is given by $v_{\text {esc }} \approx 11 \mathrm{~km} / \mathrm{s}$. If enough positive mirror charge is accumulated to overcome the Earth's gravitational attraction, then $v_{\text {esc,He }}^{2}<0$. In this case, getting stuck inside the Earth is necessary for capture. To quantify this, we define $v_{\text {cap }}$ as the maximum velocity an infalling mirror helium nucleus can have at the time it enters the Earth if it is to be captured by soft scatterings. Substituting $E_{k}(0)=\frac{1}{2} m_{X} v_{\text {cap }}^{2}, E_{k}\left(R_{\text {Earth }}\right)=\max \left[0, \frac{1}{2} m_{X} v_{\text {esc,He }}^{2}\right]$ into eq. (A.3) yields

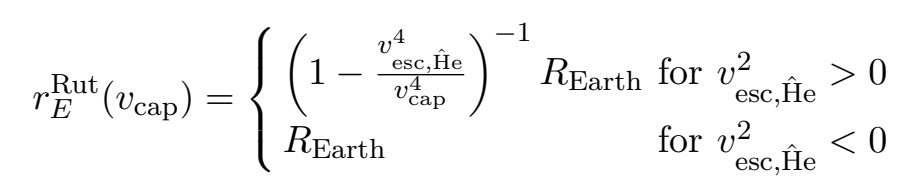

For $\epsilon \approx 10^{-10}$, in the absence of any accumulated mirror charge on the Earth, $v_{\text {cap }}^{2} \approx$ $(14 \mathrm{~km} / \mathrm{s})^{2}$, while for $\epsilon \approx 10^{-11}, v_{\text {cap }}$ is very close to $v_{\text {esc }}$, meaning that only nuclei much slower than $11 \mathrm{~km} / \mathrm{s}$ far away from the Earth get captured. 
As an $\hat{\mathrm{He}}{ }^{++}$nucleus falls towards the Earth, it gains kinetic energy $\frac{1}{2} m_{\hat{\mathrm{He}}} v_{\mathrm{esc}, \mathrm{H} \text { e }}^{2}$, where $v_{\text {esc, } \hat{H e}} \approx 11 \mathrm{~km} / \mathrm{s}$ in the absence of any accumulated mirror charge. Therefore, if far away from the Earth the $\hat{\mathrm{He}}^{++}$initially has velocity $v<\sqrt{v_{\text {cap }}^{2}-v_{\text {esc }}^{2}}$, it will become bound to the Earth. The capture rate of mirror helium from soft scatterings can therefore be estimated as $\sim n_{\hat{\mathrm{He}}}^{F} \pi R_{\text {Earth }}^{2}\left\langle v_{\hat{\mathrm{He}}}\right\rangle$, leading to a capture rate per unit volume in the Earth of

$$
n_{\hat{\mathrm{He}}}^{F} R_{\text {cap }, \hat{\mathrm{He}}}^{<v_{\text {cap }}} \sim n_{\hat{\mathrm{He}}}^{F} R_{\text {Earth }}^{-1}\left\langle v_{\hat{\mathrm{He}}}\right\rangle .
$$

Here $\left\langle v_{\hat{\mathrm{He}}}\right\rangle$ represents the average speed, far away from the Earth, of incoming mirror particles that can be captured

$$
\left\langle v_{\hat{\mathrm{He}}}\right\rangle=\int_{v_{\min }}^{\sqrt{v_{\mathrm{cap}}^{2}-v_{\mathrm{esc}, \hat{\mathrm{He}}}^{2}}} d v v f_{\hat{\mathrm{He}}}(v) .
$$

Here $f_{\hat{\mathrm{He}}}(v)$ is the local velocity distribution of mirror helium in the Earth frame. The lower limit of integration $v_{\min }=0$ as long as $v_{\text {esc, He }}^{2}>0$. For $v_{\text {esc, } \hat{H e}}^{2}<0$, only particles above a certain initial speed can even reach the Earth surface, and so $v_{\min }=\sqrt{-v_{\text {esc, }, \hat{\mathrm{He}}}^{2}}$.

While capture of slow mirror helium nuclei proceeds via multiple soft scatterings, capture of $\mathrm{He}^{++}$that enter the Earth with speed $v>v_{\text {cap }}$ can still proceed through hard scatterings. In this case we estimate the capture rate by determining the probability of having a single scattering process that takes away a significant fraction of the energy of the mirror helium nucleus.

In our estimate of the capture rate from hard scattering, we assume that the energy transfer between the incoming $\hat{H}$ e and the SM nucleus in the Earth is always maximal. This is a conservative assumption, since it overestimates the true capture rate and the resulting suppression in the direct detection signal. For a fixed mass $m_{T}$ of the target nucleus, kinematics places an upper limit on the velocities of mirror helium nuclei that can be captured through a single scattering. In particular, only mirror helium particles with velocity $v<v_{\max }=v_{\text {esc, } \mathrm{He}}\left(m_{\hat{\mathrm{He}}}+m_{T}\right) /\left|m_{\hat{\mathrm{He}}}-m_{T}\right|$ when they enter the Earth have a chance of getting captured. Here $v_{\text {esc,He }}$ is the escape velocity at the surface of the Earth. It takes value $11 \mathrm{~km} / \mathrm{s}$ in the absence of any accumulated mirror charge but is reduced if the Earth has a net charge.

We find that $\hat{H}^{++}$capture via single hard scatterings is also dominated by oxygen. We compare the scattering length $\ell \sim\left(\sigma_{X T} n_{T}\right)^{-1}$ to the Earth's radius, since we expect to have only one chance to scatter and capture the particle. The mirror capture rate per unit volume arising from single hard scatterings can then be estimated as

$$
n_{\hat{\mathrm{He}} \mathrm{e}}^{F} R_{\text {cap }, \hat{\mathrm{He}}}^{>v_{\text {cap }}} \sim n_{\hat{\mathrm{He}}}^{F}\left\langle\sigma_{\hat{\mathrm{He}} \mathrm{O}} v_{\hat{\mathrm{He}}}\right\rangle n_{\mathrm{O}},
$$

where

$$
\left\langle\sigma_{\hat{\mathrm{He}} \mathrm{O}} v_{\hat{\mathrm{He}}}\right\rangle=\int \frac{\sqrt{v_{\mathrm{max}}^{2}-v_{\mathrm{esc}, \hat{\mathrm{He}}}^{2}}}{\sqrt{v_{\mathrm{cap}}^{2}-v_{\mathrm{esc}, \hat{\mathrm{He}}}^{2}}} d v \sigma_{\hat{\mathrm{He}} \mathrm{O}} v f_{\hat{\mathrm{He}}}(v), \quad \sigma_{\hat{\mathrm{HeO}}} \approx \frac{4 \pi \alpha^{2} \epsilon^{2}}{m_{\hat{\mathrm{He}}}^{2}\left(v^{2}+v_{\mathrm{esc}, \hat{\mathrm{He}}}^{2}\right)^{2}} .
$$



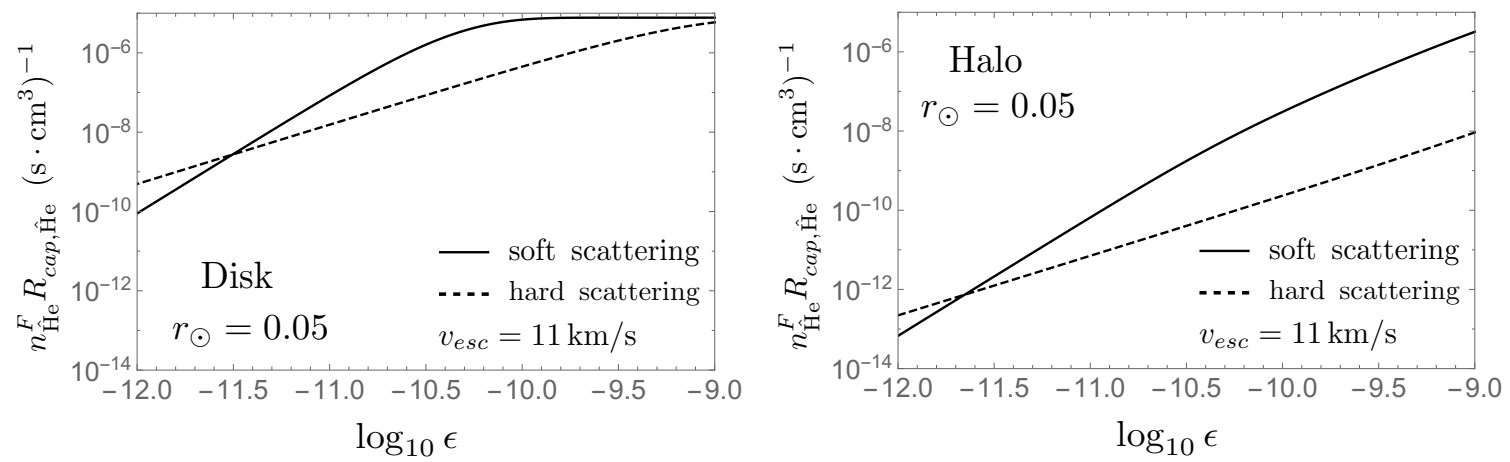

Figure 7. Mirror helium capture rates from multiple soft scattering (solid) and single hard scattering (dashed) processes as a function of the kinetic mixing parameter $\epsilon$ for $r_{\odot}=0.05$. This plot assumes that the net mirror charge of the Earth is small, so that its effect on the escape velocities of mirror particles is negligible.

The capture rates of mirror helium arising from soft and hard scattering as a function of $\epsilon$ are shown in figure 7 for both halo and disk distributions. We see that capture by soft scattering dominates for $\epsilon \gtrsim 10^{-12}$.

In most of our estimates, we neglect self-capture of incoming mirror helium nuclei by the captured He population already inside the Earth. It is important to understand when this is a valid approximation. Self-capture is dominated by multiple soft scatterings. We can obtain an estimate for the penetration depth of an incoming mirror helium nucleus due to interactions with captured Ĥe by substituting $n_{T} \rightarrow N_{\hat{\mathrm{He}}} /\left(\frac{4}{3} \pi R_{\text {Earth }}^{3}\right), m_{T, X} \rightarrow m_{\hat{\mathrm{He}} \mathrm{e}}$, $Q_{T, X} \rightarrow 2, Z \rightarrow 2, \epsilon \rightarrow 1$ into eq. (A.4),

$$
L_{\hat{\mathrm{He}}-\hat{\mathrm{He}}} \sim R_{\mathrm{Earth}}\left(\frac{2 \cdot 10^{31}}{N_{\hat{\mathrm{He}}}}\right)\left(\frac{v_{X}}{20 \mathrm{~km} / \mathrm{s}}\right)^{4} .
$$

In order for our estimates neglecting self capture to be trustworthy, capture must be dominated by scattering off SM particles in the Earth,

$$
L_{\hat{\mathrm{He}}-\hat{\mathrm{He}}} \gg r_{E}^{\mathrm{Rut}}
$$

This corresponds to the condition,

$$
N_{\hat{\mathrm{He}}} \ll 10^{31}\left(\frac{\epsilon}{10^{-10}}\right)^{2}
$$

which specifies the regime of validity of our estimates. It is also interesting to consider the regime of runaway self-capture, where $L_{\hat{\mathrm{He}}-\hat{\mathrm{He}}} \sim R_{\text {Earth }}$ and most incoming mirror helium nuclei get captured. Taking $v_{X} \sim v_{\odot, \hat{H e}}$ we see that we enter this regime if $N_{\hat{\mathrm{He}}} \gtrsim 10^{30}\left(10^{34}\right)$ for the disk (halo) distributions. 


\section{A.1.2 Capture and evaporation of mirror electrons}

In this subsection we discuss the capture and evaporation of mirror electrons in the halo and disk scenarios. Our analysis shows that the capture of mirror electrons has a negligible effect on direct detection for small values of the kinetic mixing, $\epsilon \lesssim 10^{-11}$.

In the halo distribution, mirror electrons have an average velocity $v_{\odot, \hat{e}} \sim 7 \times 10^{3} \mathrm{~km} / \mathrm{s}$, corresponding to a kinetic energy of $\sim 400 \mathrm{eV}$. This is comparable to the binding energy of inner shell electrons in atoms. Therefore capture primarily arises from the scattering of mirror electrons with inner shell electrons. This process is inelastic since the atom is either left in an excited state or the electron is simply ejected from the atom leaving behind an ion, and may be accompanied by the emission of additional (mirror) photons. Although this process can result in the mirror electron losing enough energy to be captured, the phase space for capture is very limited since the velocity of the incoming mirror electron is orders of magnitude larger than the escape velocity from the Earth.

Since iron is the most abundant element in the Earth, for concreteness we will focus on scattering from iron atoms in the Earth's core. A process that can result in the loss of the required amount of energy involves $\hat{e}$ kicking out electrons from the $2 p$ state of an iron atom $(\Delta E \approx 700 \mathrm{eV})$. We can therefore estimate the scattering length by assuming each iron atom has 6 useful electrons for the capture process. A detailed calculation of the capture that takes into account the details of the atomic structure is beyond the scope of this work. We will instead place an upper bound on the number of captured mirror electrons, where the limit is obtained by assuming that $\hat{e}$ is always captured in scatterings with $2 p$ electrons regardless of the actual momentum transfer. We will later show that even if the bound is saturated the number of mirror electrons in the Earth is still too small to affect the direct detection signals. From the density of iron inside the core $\approx 13 \mathrm{~g} \mathrm{~cm}^{-3}$, we can place a lower bound on the capture length as,

$$
\ell_{\hat{e}, \mathrm{Halo}}^{\text {cap }} \gtrsim\left(\sigma_{\hat{e} e} 6 n_{\mathrm{Fe}}\right)^{-1} \sim 10^{8} \mathrm{~km}\left(\frac{\epsilon}{10^{-9}}\right)^{-2} .
$$

We now turn our attention to the disk distribution. Here mirror electrons have velocities of order $v_{0, \hat{e}} \sim 6 \times 10^{2} \mathrm{~km} / \mathrm{s}$, corresponding to kinetic energies of order $3 \mathrm{eV}$. This energy is close to the thickness of the valence band of iron below the Fermi surface. Therefore, an efficient scattering process involves $\hat{e}$ kicking out an electron from this band. Nevertheless the phase space for capture is limited, since the velocity of the incoming mirror electrons is still much more than the escape velocity from the Earth. Again, a calculation of the capture that takes into account the precise dispersion relation of the electrons inside the metal is beyond the scope of this work. We instead estimate the scattering length by rescaling the mean free path of the electrons in iron at room temperature [169], for which the associated energy transfer between electrons is not far from the $\mathrm{eV}$ scale. We again limit ourselves to placing an upper bound on the number density of captured mirror electrons, where the bound is reached if $\hat{e}$ is always captured in scatterings with conduction electrons regardless of the actual momentum transfer. We will later show that even if the bound is saturated, the number of captured mirror electrons is too small to affect the signal. The lower bound 
on the capture length of disk mirror electrons is given by ${ }^{16}$

$$
\ell_{\hat{e}, \text { Disk }}^{\text {cap }} \gtrsim 10^{7} \mathrm{~km}\left(\frac{\epsilon}{10^{-9}}\right)^{-2} \text {. }
$$

From the lower bound on the capture length, we can obtain an upper bound on the capture rate of mirror electrons per unit volume in the Earth in the halo and disk cases,

$$
n_{\hat{e}}^{F} R_{\text {cap }, \hat{e}} \lesssim n_{\hat{e}}^{F} R_{\text {core }}^{-1} v_{\odot, \hat{e}}\left(\frac{R_{\text {core }}}{\ell_{\hat{e}}^{\text {cap }}}\right)=n_{\hat{e}}^{F}\left(\frac{v_{\odot, \hat{e}}}{\ell_{\hat{e}}^{\text {cap }}}\right)
$$

After capture, the $\hat{e}$ has a velocity $v \lesssim v_{\text {esc }, \hat{e}}$, where $v_{\text {esc }, \hat{e}}$ is the escape velocity at the Earth's core. We expect that the mirror electrons will tend to thermalize with the matter in the Earth's core. If the net accumulated mirror charge of the Earth is small, so that the thermal velocity of the mirror electrons is larger than their escape velocity, any captured $\hat{e}$ are efficiently evaporated from the Earth. It is only if the escape velocity of the mirror electrons is greater than or comparable to their thermal velocity that there is any significant accumulation of $\hat{e}$.

An efficient evaporation process for the $\hat{e}$ involves scattering off conduction band electrons in metals. We will once again focus on iron. When scattering off mirror electrons, electrons in the conduction band can impart energy that is comparable to the width of the valence band $(\sim 0.6 \mathrm{eV})$ that corresponds to the iron temperature inside the Earth's core $\left(T_{\text {Earth }} \sim 4000-6000 \mathrm{~K}\right)$. This means that a captured mirror electron will be promptly expelled by collision with a conduction electron unless the net positive mirror charge of the Earth is so large that the escape velocity of $\hat{e}$ at the Earth's core is greater than the thermal velocity $v_{t h, \hat{e}}$, which is of order $260 \mathrm{~km} / \mathrm{s}$. We can estimate the mean free path for $\hat{e}$ evaporation by rescaling the mean free path in eq. (A.14) after accounting for the change of the $\hat{e}-e$ scattering cross section with the energies of the incoming and outgoing particles. The Fermi velocity of electrons in iron, $v_{F} \approx 2 \times 10^{3} \mathrm{~km} / \mathrm{s}$ in iron, is much greater than $v_{t h, \hat{e}}$. Then the scattering cross section for a fast moving electron with velocity $v_{F}$ to evaporate a captured mirror electron of velocity $v_{t h, \hat{e}}$ by injecting a recoil energy $E_{R} \gtrsim \frac{1}{2} m_{\hat{e}} v_{\text {esc, }, \hat{e}}^{2}$ can be estimated as

$$
\sigma_{e v p} \approx \frac{4 \pi \alpha^{2} \epsilon^{2}}{m_{\hat{e}}^{2} v_{F}^{2} v_{\mathrm{esc}, \hat{e}}^{2}} \propto\left(m_{\hat{e}}^{2} v_{F}^{2} v_{\mathrm{esc}, \hat{e}}^{2}\right)^{-1}
$$

From this cross section, for $v_{\text {esc }, \hat{e}} \lesssim v_{t h, \hat{e}}$, we obtain an upper bound for the mean free path for evaporation,

$$
\ell_{\hat{e}, \text { Disk \& Halo }}^{\text {eva }} \lesssim 10^{-1} \ell_{\hat{e}, \text { Disk }}^{\text {cap }}
$$

\footnotetext{
${ }^{16}$ Since the iron in the earth's core is at a higher temperature and pressure, the mean free path is expected to be somewhat shorter in the core, leading to a smaller capture length. However, as we show below, a shorter mean free path also corresponds to more efficient evaporation of mirror electrons. Therefore, as long as the mean free paths for capture and evaporation are much larger than the Earth's radius, the shorter $\ell_{\hat{e}, D i s k}^{\text {cap }}$ will not significantly affect the equilibrium $\hat{e}$ abundance in the disk case and will only decrease the abundance in the halo case.
} 
In the absence of significant charge accumulation, the evaporation rate of mirror electrons per unit volume is given by

$$
n_{\hat{e}}^{C} R_{e v p, \hat{e}} \sim n_{\hat{e}}^{C}\left(\frac{v_{F}}{\ell_{\hat{e}}^{\text {eva }}}\right) .
$$

When the system is in equilibrium $n_{\hat{e}}^{F} R_{\text {cap }, \hat{e}}=n_{\hat{e}}^{C} R_{e v p, \hat{e}}$. From the upper bound on the capture rate we can obtain an upper bound on the total number of captured mirror electrons,

$$
\left.N_{\hat{e}} \lesssim n_{\hat{e}}^{F}\left(\frac{v_{\odot, \hat{e}}}{v_{F}}\right)\left(\frac{\ell_{\hat{e}}^{\text {eva }}}{\ell_{\hat{e}}^{\text {cap }}}\right) R_{\text {core }}^{3} \sim 10^{23} \text { (Disk, Halo }\right) .
$$

This bound is valid for $v_{\text {esc }, \hat{e}} \lesssim v_{t h, \hat{e}}$. However, since in this regime the velocity of incoming mirror electrons is still much greater than the escape velocity, $v_{\odot, \hat{e}} \gg v_{\text {esc }, \hat{e}}$, the phase space for capture is very limited and so the actual number of captured electrons is expected to be several orders of magnitude less than this upper bound.

If the Earth acquires a large net positive charge the evaporation rate is exponentially suppressed because of the much larger velocity needed to escape the Earth. Under the assumption that the mirror electrons thermalize with the matter in the Earth's core, eq. (A.18) generalizes to

$$
n_{\hat{e}}^{C} R_{e v p, \hat{e}} \sim n_{\hat{e}}^{C}\left(\frac{v_{F}}{\ell_{\hat{e}}^{\text {eva }}}\right) \exp \left(-\frac{m_{\hat{e}} v_{\text {esc }, \hat{e}}^{2}}{2 T_{\text {Earth }}}\right) .
$$

In equilibrium we get the upper bound,

$$
\begin{aligned}
N_{\hat{e}} & \lesssim n_{\hat{e}}^{F}\left(\frac{v_{\odot, \hat{e}}}{v_{F}}\right)\left(\frac{\ell_{\hat{e}}^{\text {eva }}}{\ell_{\hat{e}}^{\text {cap }}}\right) \exp \left(\frac{m_{\hat{e}} v_{\text {esc }, \hat{e}}^{2}}{2 T_{\text {Earth }}}\right) R_{\text {core }}^{3}, \\
& \left.\sim \exp \left(\frac{m_{\hat{e}} v_{\text {esc }, \hat{e}}^{2}}{2 T_{\text {Earth }}}\right) \times 10^{23} \text { (Disk, Halo }\right),
\end{aligned}
$$

for the disk and halo cases. This bound is only saturated when the captured charge is large enough that the escape velocity is greater than or of order the velocity of incoming mirror electrons, $v_{\text {esc }, \hat{e}} \gtrsim v_{\odot, \hat{e}}$. However, for $\epsilon \lesssim 10^{-11}$, we shall see that the number of captured mirror nuclei is too small to affect the evaporation of mirror electrons.

Just as for mirror helium capture, we now determine the condition for mirror electron self-capture to play a significant role. Assuming that the captured mirror electrons in the Earth are roughly uniformly distributed, the mean free path for scattering of incoming mirror electrons with the captured population can be estimated as,

$$
L_{\hat{e}-\hat{e}} \sim\left(\frac{4 \pi \alpha^{2}}{m_{\hat{e}}^{2} v_{\odot, \hat{e}}^{4}} n_{\hat{e}}^{C}\right)^{-1} \sim\left(\frac{10^{23}}{N_{\hat{e}}}\right) \times\left\{\begin{array}{l}
10^{13} \mathrm{~km} \text { (disk) } \\
10^{17} \mathrm{~km} \text { (halo) }
\end{array}\right.
$$

Requiring self capture to be negligible compared to scattering off SM electrons in the Earth corresponds to the conditions $L_{\hat{e}-\hat{e}} \gg \ell_{\hat{e}, \text { Halo }}^{\text {cap }}, \ell_{\hat{e}, \text { Disk }}^{\text {cap }}$. Using eqs. (A.13) and (A.14), this translates to an upper bound on the number of captured mirror electrons,

$$
N_{\hat{e}} \ll\left(\frac{\epsilon}{10^{-9}}\right)^{2} \begin{cases}10^{29} & \text { (disk) } \\ 10^{32} & \text { (halo) }\end{cases}
$$


We will later see that self capture is never important for kinetic mixings $10^{-12} \lesssim \epsilon \lesssim 10^{-9}$ where capture is non-negligible. (Capture is negligible for much smaller kinetic mixings.) We are even further away from the runaway self-capture regime, corresponding to $L_{\hat{e}-\hat{e}} \sim R_{\text {Earth }}$, which is only reached for $N_{\hat{e}} \gtrsim 10^{32}\left(10^{36}\right)$ assuming a disk (halo) distribution.

\section{A.1.3 Screening and the escape velocity}

The Earth will eventually accumulate more mirror helium nuclei than mirror electrons and acquire a net positive charge. This net charge is screened by the ambient mirror plasma within a short distance of the Earth's surface. We discuss this phenomenon here, since it can have a significant impact on the capture and evaporation processes. To simplify the discussion, we neglect the effects of gravity on the mirror electric potential. We have verified that including these effects does not alter our conclusions.

The mirror electric potential $\phi(r)$ close to the Earth follows the Poisson equation,

$$
\nabla^{2} \phi(r)=-\sum_{i} q_{i} n_{i}^{C}(r)-\sum_{i} q_{i} n_{i}^{S}(r)
$$

where $r$ is the distance from the Earth center, $n_{i}^{C}$ is number density of the captured mirror particle species (dominated by $i=\hat{\mathrm{He}}$ ), and $n_{i}^{S}$ is the free number density of the mirror particle species $i$ with mirror charge $q_{i}$. This ambient but uncaptured density near the Earth reacts to the accumulated charge to screen it, and asymptotes to the unperturbed free mirror number densities $n_{i}^{F}$ far away from the Earth. Solving eq. (A.24) in generality is quite complicated, since the free charge and captured charge distribution (which adjusts itself through diffusion in the Earth, section A.1.4) are themselves functions of the potential. Here we will derive a simplified solution that is conservative in the sense that it underestimates mirror helium evaporation and therefore overestimates the equilibrium accumulated Heabundance and its effects on direct detection. We start by expressing $n_{i}^{S}$ as a function of $\phi$, since the free mirror-charge distributions near the Earth adjust quickly to the existence of any mirror-electric potential. This allows us to solve for $\phi$ within our pessimistic but simplified assumptions.

It is convenient to parametrize the potential $\phi$ in terms of its contribution to the escape velocity of a particle species $i$ at a radius $r$ from the center of the Earth,

$$
v_{\mathrm{esc}, i}^{2}(r)=-\frac{2\left(e q_{i} \phi(r)+\phi_{g r}(r)\right)}{m_{i}} .
$$

As noted above we will ignore gravity in this discussion, i.e. $\phi_{g r}=0$. Recall that $v_{\mathrm{esc}, i}^{2}<0$ signifies a repulsive rather than attractive force on the particle. To determine $n_{i}^{S}(r)$, we consider particle trajectories that approach the Earth from far away. By calculating the fraction of this incoming flux that reaches a distance $r$ from the center of the Earth, we can obtain an expression for $n_{i}^{S}(r)$.

We first consider all the trajectories that stretch from some large but finite $r_{\infty}$ to $r$, where $r_{\infty}$ will be taken to infinity at the end. We denote the speeds of the particles at $r_{\infty}$ and $r$ by $v_{\infty}$ and $v$ respectively. We employ the coordinate system shown in figure 8 , in 

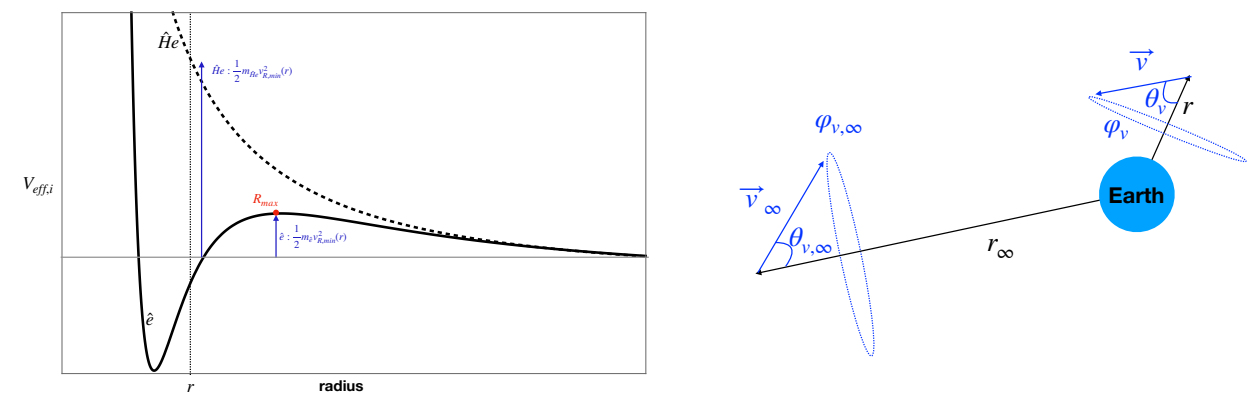

Figure 8. On the left is an illustration of the one dimensional effective potentials for positively and negatively charged mirror particles induced by a net captured positive charge inside the Earth (shown for $\hat{\mathrm{He}}^{++}$as a dashed line and for $\hat{\mathrm{e}}^{-}$as a solid line). The one dimensional effective potential for negatively charged particles exhibits a local maximum where the repulsive force from angular momentum exactly balances the mirror electrostatic attraction. In this case for an incoming particle to reach any point deeper than $R_{\max }$, the location of the maximum of the barrier, the radial kinetic energy at infinity must be greater than the height of the barrier at $R_{\max }$. For the case of positively charged particles no such barrier exists. Then, to reach any point $r$, it suffices for the radial kinetic energy of the incoming particle at infinity to be greater than the effective potential at $r$. On the right we illustrate our choice of coordinates for the velocity distribution.

which the expressions for the radial and tangential velocities at $r_{\infty}$ and $r$ take the form,

$$
\begin{array}{rlrl}
v_{r, \infty} & =v_{\infty} \cos \theta_{v, \infty}, & v_{r} & =v \cos \theta_{v} \\
v_{\tan , \infty} & =\sqrt{v_{\infty}^{2}-v_{r, \infty}^{2}}, \quad v_{\tan }=\sqrt{v^{2}-v_{r}^{2}}
\end{array}
$$

For each species $i \in(\hat{H} e, \hat{H}, \hat{e})$, these quantities are related by energy and angular momentum conservation,

$$
v^{2}-v_{\mathrm{esc}, i}^{2}=v_{\infty}^{2}, r^{2}\left(v^{2}-v_{r}^{2}\right)=r_{\infty}^{2}\left(v_{\infty}^{2}-v_{r, \infty}^{2}\right)
$$

where we have assumed that the escape velocity at $r_{\infty}$ is negligible.

The total radial flux of particles that enter a sphere of radius $r$ centered at the Earth with speeds between $v$ and $v+d v$ and radial velocities between $v_{r}$ and $v_{r}+d v_{r}$ is equal to the radial flux of particles which enter a sphere of radius $r_{\infty}$ with speeds between $v_{\infty}$ and $v_{\infty}+d v_{\infty}$ and radial velocities between $v_{r, \infty}$ and $v_{r, \infty}+d v_{r, \infty}$, provided that the velocities satisfy the relations in eq. (A.27). (This assumes that there is no potential barrier in between $r_{\infty}$ and $r$ that affects the flow of particles between these two points.) We take $f_{i}(\vec{r}, \vec{v})$ to be the number of mirror particles of species $i$ that have positions lying within a volume element $d^{3} \vec{r}$ about $\vec{r}$ and velocities lying within a velocity space element $d^{3} \vec{v}$ about $\vec{v}$. The spherical symmetry of the problem implies that $f_{i}(\vec{r}, \vec{v})$ depends only on the radial coordinate $r$, the projection of the velocity in the radial direction $v_{r}$ and the speed $v$, $f_{i}(\vec{r}, \vec{v})=f_{i}\left(r, v, v_{r}\right)$. This allows us to write

$$
v_{r} \frac{d v_{r}}{v} v^{2} d v \int f_{i}\left(r, v, v_{r}\right) r^{2} d \Omega d \phi_{v}=v_{r, \infty} \frac{d v_{r, \infty}}{v_{\infty}} v_{\infty}^{2} d v_{\infty} \int f_{i}\left(r_{\infty}, v_{\infty}, v_{r, \infty}\right) r_{\infty}^{2} d \Omega_{\infty} d \phi_{v, \infty}
$$


If we neglect capture inside the Earth, an identical relation holds for particles flowing outward from $r$ ro $r_{\infty}$. Integrating over all $v_{r}$ from $v_{r}=-v$ to $v_{r}=v$, and $\phi_{v}$ from 0 to $2 \pi$ we can find the speed distribution of incoming particles $f_{i}(\vec{r}, v)$ about the point $\vec{r}$,

$$
f_{i}(\vec{r}, v)=\int f_{i}(\vec{r}, \vec{v}) v_{r} \frac{d v_{r}}{v} v^{2} d \phi_{v}
$$

The speed distribution is related to the number density as $\int d v f_{i}(\vec{r}, v)=n_{i}(\vec{r})$. Because of the radial symmetry of the problem, $f_{i}(\vec{r}, v)=f_{i}(r, v)$. Performing the integration we obtain for the speed distribution at radius $r$,

$$
\begin{aligned}
f_{i}(r, v) & =\frac{1}{4 \pi} \int f_{i}\left(r, v, v_{r}\right) v^{2} d \Omega d \phi_{v} \frac{d v_{r}}{v}= \\
& =\frac{1}{4 \pi} \int f_{i}\left(r_{\infty}, v_{\infty}, v_{r, \infty}\right) \frac{r_{\infty}^{2}}{r^{2}} \frac{v_{r, \infty}}{v_{r}} v_{\infty}^{2} \gamma\left(\vec{r}_{\infty}, r, \vec{v}_{\infty}\right) d \Omega_{\infty} d \phi_{v, \infty} \frac{d v_{\infty}}{d v} \frac{d v_{r, \infty}}{v_{\infty}}
\end{aligned}
$$

where we have used eq. (A.28) in the last line. Here $\gamma\left(\vec{r}_{\infty}, r, \vec{v}_{\infty}\right)$ is defined to be equal to one if a trajectory starting at $\vec{r}_{\infty}$ with velocity $\vec{v}_{\infty}$ reaches radius $r$ and zero if that is not the case. This factor is included to account for the possibility that there is a potential barrier somewhere between $r$ and $r_{\infty}$ that prevents the flow of particles between them if their energy is too low. Spherical symmetry ensures that

$$
\gamma\left(\vec{r}_{\infty}, r, \vec{v}_{\infty}\right)=\gamma\left(v_{\infty}, v_{r, \infty}, r_{\infty}, r\right)
$$

This allows us to rewrite eq. (A.30) as,

$$
f_{i}(r, v)=\frac{r_{\infty}^{2}}{r^{2}} \frac{d v_{\infty}}{d v} f_{i}\left(r_{\infty}, v_{\infty}\right) \int \frac{v_{r, \infty}}{v_{r}} \gamma\left(v_{\infty}, v_{r, \infty}, r_{\infty}, r\right) \frac{d v_{r, \infty}}{v_{\infty}}
$$

where we have assumed that $r_{\infty}$ is large enough that the velocity distribution there is approximately isotropic.

To determine $\gamma\left(v_{\infty}, v_{r, \infty}, r_{\infty}, r\right)$ we consider the equivalent one dimensional problem with the effective potential

$$
V_{\mathrm{eff}, i}(r)=-\frac{1}{2} m_{i} v_{\mathrm{esc}, i}^{2}(r)+\frac{1}{2} \frac{L^{2}}{m_{i} r^{2}}
$$

where $L$ is the angular momentum. For mirror nuclei the force is always repulsive and so the condition that a particle with radial velocity $v_{r, \infty}$ reaches a point a distance $r$ from the center of the Earth is given by,

$$
\frac{1}{2} m_{i} v_{r, \infty}^{2} \geq V_{\mathrm{eff}, i}(r)
$$

This can be rewritten as

$$
v_{r, \infty}^{2} \geq v_{r, \min }^{2} \equiv v_{\infty}^{2}-\frac{r^{2}}{r_{\infty}^{2}}\left(v_{r, \infty}^{2}+v_{\mathrm{esc}, i}^{2}(r)\right) \rightarrow v_{\infty}^{2}-\frac{r^{2}}{r_{\infty}^{2}}\left(v_{\infty}^{2}+v_{\mathrm{esc}, i}^{2}(r)\right)
$$


where $i \in \hat{\mathrm{He}}, \hat{\mathrm{H}}$ and in the last line we have taken the $r_{\infty} \rightarrow \infty$ limit. We integrate eq. (A.32) with respect to $v_{r, \infty}$ with the lower limit of integration set to $v_{r, \min }$ and find that for the nuclei,

$$
f_{i}(r, v)=\frac{v^{2}}{v_{\infty}^{2}} f_{i}\left(r_{\infty}, v_{\infty}\right)=\frac{v^{2}}{v^{2}-v_{\mathrm{esc}, i}^{2}} f_{i}\left(r_{\infty}, \sqrt{v^{2}-v_{\mathrm{esc}, i}^{2}}\right) .
$$

For the mirror electrons the situation is more complicated since the force is attractive, so that the effective potential can acquire a local maximum as shown in figure 8 . If the trajectory is to reach a distance $r$ from the center of the Earth the radial component of the kinetic energy must be greater than or equal to the effective potential at all points for which the radial coordinate $R \geq r$,

$$
\frac{1}{2} m_{i} v_{r, \infty}^{2} \geq \max _{R>r} V_{\mathrm{eff}, i}(R)
$$

We can rewrite this condition as,

$$
v_{\infty}^{2}-v_{r, \infty}^{2} \leq \min _{R>r} \frac{R^{2}}{r_{\infty}^{2}}\left(v_{r, \infty}^{2}+v_{\mathrm{esc}, \hat{e}}^{2}(R)\right) \rightarrow \min _{R>r} \frac{R^{2}}{r_{\infty}^{2}}\left(v_{\infty}^{2}+v_{\mathrm{esc}, \hat{e}}^{2}(R)\right)
$$

where the last step is valid in the $r_{\infty} \rightarrow \infty$ limit. For any given $v_{\infty}$ we can find the radius $R_{\max }=R_{\max }\left(v_{\infty}\right)$ that maximizes the right hand side in eq. (A.38) (without the restriction $R>r)$,

$$
\partial_{r} v_{\mathrm{esc}, \hat{e}}^{2}\left(R_{\max }\right) R_{\max }+2\left(v_{\infty}^{2}+v_{\mathrm{esc}, \hat{e}}^{2}\left(R_{\max }\right)\right)=0 .
$$

We now can see that $\gamma\left(v_{\infty}, v_{r, \infty}, r_{\infty}, r\right)=1$ for the electrons when

$$
v_{r, \infty}^{2} \geq v_{r, \text { min }}^{2} \equiv \begin{cases}v_{\infty}^{2}-\frac{R_{\max }^{2}}{r_{\infty}^{2}}\left(v_{\infty}^{2}+v_{\mathrm{esc}, \hat{e}}^{2}\left(R_{\max }\right)\right) & \text { for } r<R_{\max }\left(v_{\infty}\right) \\ v_{\infty}^{2}-\frac{r^{2}}{r_{\infty}^{2}}\left(v_{\infty}^{2}+v_{\mathrm{esc}, \hat{e}}^{2}(r)\right) & \text { for } r \geq R_{\max }\left(v_{\infty}\right)\end{cases}
$$

Here $R_{\max }\left(v_{\infty}\right)$ corresponds to the position of the maximum of the angular momentum barrier for an initial speed $v_{\infty}$ at infinity. Integrating eq. (A.32) with respect to $v_{r, \infty}$, with $v_{r, \min }$ as the lower limit of integration, we find for the mirror electron distribution,

$$
\frac{f_{\hat{e}}(r, v)}{f_{\hat{e}}\left(r_{\infty}, v_{\infty}\right)}= \begin{cases}\frac{v}{v_{\infty}^{2}}\left(v-\operatorname{Re} \sqrt{v^{2}-\frac{R_{\max }^{2}}{r^{2}}\left(v^{2}-v_{\mathrm{esc}, \hat{e}}^{2}(r)+v_{\mathrm{esc}, \hat{e}}^{2}\left(R_{\max }\left(v_{\infty}\right)\right)\right)}\right) & r<R_{\max }\left(v_{\infty}\right) \\ \frac{v^{2}}{v_{\infty}^{2}} & r \geq R_{\max }\left(v_{\infty}\right)\end{cases}
$$

In order to simplify the discussion we first consider a toy model in which the distribution $f_{i}\left(r_{\infty}, v_{\infty}\right)$ takes the form of a delta function with speed $\frac{3}{2} v_{\odot, i}$ for each species. As we shall see, this simpler case captures the main features of the more complicated Maxwell-Boltzmann distribution. With this we find that the number density of electrons is given by

$$
\frac{n_{\hat{e}}^{S}(r)}{n_{\hat{e}}^{F}}=\sqrt{1+\frac{3 v_{\mathrm{esc}, \hat{e}}^{2}}{2 v_{\odot, \hat{e}}^{2}}}- \begin{cases}\operatorname{Re} \sqrt{1+\frac{3 v_{\mathrm{esc}, \hat{e}}^{2}}{2 v_{\odot, \hat{e}}^{2}}-\frac{R_{\max }^{2}}{r^{2}}\left(1+\frac{3 v_{\mathrm{esc}, \hat{e}}^{2}\left(R_{\max }\right)}{2 v_{\odot, \hat{e}}^{2}}\right)} & r<R_{\max } \\ 0 \quad & r \geq R_{\max }\end{cases}
$$


while for the nuclei $i \in \hat{\mathrm{H} e}, \hat{\mathrm{H}}$ we obtain,

$$
\frac{n_{i}^{S}(r)}{n_{i}^{F}}=\sqrt{1+\frac{3 v_{\mathrm{esc}, i}^{2}}{2 v_{\odot, i}^{2}}}
$$

Inserting this into the Poisson equation for the potential $\phi(r)$, we can rewrite it in terms of just the captured number density and the free number density far away from the Earth,

$$
\begin{aligned}
-\lambda_{D}^{2} \nabla^{2} \varphi_{\mathrm{esc}}(r)= & -\beta-\alpha_{H e} \sqrt{1-2 \varphi_{\mathrm{esc}}}-\alpha_{H} \sqrt{1-\varphi_{\mathrm{esc}}} \\
& -\alpha_{e}\left(\sqrt{1+\varphi_{\mathrm{esc}}}-\left\{\begin{array}{ll}
\operatorname{Re} \sqrt{1+\varphi_{\mathrm{esc}}-\frac{R_{\max }^{2}}{r^{2}}\left(1+\varphi_{\mathrm{esc}}\left(R_{\max }\right)\right)} r<R_{\max } \\
0 & r>R_{\max }
\end{array}\right)\right.
\end{aligned}
$$

We have defined a dimensionless potential

$$
\varphi_{\mathrm{esc}}(r)=\frac{e \phi}{T_{\text {mirror }}}=-\frac{3 v_{\mathrm{esc}, i}^{2}(r)}{2 q_{i} v_{\odot, i}^{2}} \quad \forall i,
$$

and introduced the dimensionless parameters,

$$
\begin{aligned}
\alpha_{i} & =\frac{q_{i} n_{i}^{F}}{\sum q_{j}^{2} n_{j}^{F}} \quad \text { for } \quad i=\hat{\mathrm{He}}, \hat{\mathrm{H}}, \hat{e}, \\
\beta(r) & =\frac{\sum q_{i} n_{i}^{C}(r)}{\sum q_{j}^{2} n_{j}^{F}} .
\end{aligned}
$$

Note that $\beta$ parametrizes the size of the captured mirror charge density relative to that of the ambient plasma far away from the Earth. Charge neutrality of the external plasma requires $\sum \alpha_{i}=0$. The Debye length $\lambda_{D}$ is a constant of the problem, defined as

$$
\lambda_{D}=\left(4 \pi \alpha \frac{\sum q_{i}^{2} n_{i}^{F}}{T_{\text {mirror }}}\right)^{-1 / 2} \sim\left(\frac{r_{\odot}}{0.05}\right)^{-1 / 2} \times\left\{\begin{array}{l}
100 \mathrm{~m} \text { (disk) } \\
1000 \mathrm{~m} \text { (halo) }
\end{array}\right.
$$

The potential must vanish as $r \rightarrow \infty$, and in fact vanishes exponentially within a Debye length of $R_{\max }$. For $r>R_{\max }$ the equation simplifies to the standard Debye-Huckel equation which has the solution,

$$
\varphi_{\mathrm{esc}}(r)=\varphi_{\mathrm{esc}}\left(R_{\max }\right) \exp \left\{-\frac{r-R_{\max }}{\lambda_{D}}\right\} \frac{R_{\max }}{r} \quad \text { for } \quad r>R_{\max } .
$$

Since $\lambda_{D}$ is at most of order a few kms and much smaller than the size of the Earth, we can work in the approximation where $\lambda_{D}=0$ and the right hand side of eq. (A.44) vanishes.

We can now insert the full solution at $r>R_{\max }$ of eq. (A.49) into eq. (A.39) to find the boundary condition at $r=R_{\max }$ for the $r<R_{\max }$ solution. This is justified because the full solution is continuous and has a continuous derivative at $r=R_{\max }$. In eq. (A.39) the derivative term dominates in the limit that $\lambda_{D} \rightarrow 0$, because the derivative of $\varphi_{\text {esc }}(r)$ in eq. (A.49) is proportional to $\varphi_{\mathrm{esc}}\left(R_{\max }\right) / \lambda_{D}$. Therefore, in this limit the boundary condition at $R_{\max }$ is simply $\varphi_{\mathrm{esc}}\left(R_{\max }\right)=0$. 
To find $\varphi_{\text {esc }}(r)$ for $r<R_{\max }$, we first study the regime of small charge accumulation, where $\beta \ll 1$. This is a good approximation for $\epsilon \leq 10^{-11}$. We will make the simplifying assumption that the potential is constant inside the Earth, and the captured mirror helium abundance has a profile that will ensure this is maintained. In reality, $\phi(r)$ must decrease with increasing $r$, since it would push the accumulated charges away from each other and towards the Earth surface. By assuming the potential to be constant, we are therefore underestimating mirror helium evaporation and overestimating the effects of accumulation. As $r \rightarrow 0$, the Re term in eq. (A.44) vanishes and we can algebraically solve with the right hand side set to zero to obtain the value of the potential near the center of the Earth,

$$
\varphi_{\mathrm{esc}}(r \rightarrow 0) \equiv \varphi_{\mathrm{esc}}^{\mathrm{Earth}}=\frac{\beta_{0}}{2 \alpha_{\hat{\mathrm{H}}}+3 \alpha_{\hat{\mathrm{He}}}} \ll 1 \quad \text { for } \quad \beta_{0} \ll 1,
$$

where $\beta_{0}$ is the value of $\beta$ at the center of the Earth. Note that $\beta(r)$ adjusts to ensure that the right hand side vanishes in eq. (A.44) while keeping

$$
\varphi_{\mathrm{esc}}(r)=\varphi_{\mathrm{esc}}^{\mathrm{Earth}} \quad \text { for } \quad r<R_{\text {Earth }} .
$$

So now we have an expression for the constant potential for $r<R_{\text {Earth }}$. We also know that the potential is zero for $r>R_{\max }>R_{\text {Earth }}$, in the $\lambda_{D} \rightarrow 0$ approximation. All that remains is to find the potential in the transition region $R_{\text {Earth }}<r<R_{\max }$, by setting the right hand side of eq. (A.44) to zero with $\beta=0$. From this we can find the value of $R_{\max }$

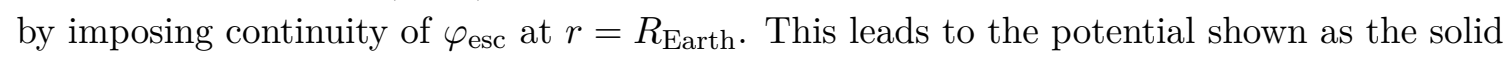
gray curve in figure 9 .

This solution has been obtained neglecting the gravitational potential of the Earth. Including the Earth's gravity leads to a modest correction to the mirror electrostatic potential which acts to compensate for the fact that the effect of gravity is larger on the positively charged mirror nuclei than on the negatively charged mirror electrons. As noted earlier, gravity has only a subdominant effect on our results.

Now we consider the realistic Maxwell-Boltzman distribution of speeds at infinity, rather than the delta function. This case is more complicated, and can only be solved numerically. Our approach is to solve recursively to find $R_{\max }$ as a function of $v_{\infty}$. This is done by discretizing the speed distribution as $v_{\infty, k}$ and noting that $R_{\max }$ goes down as the speed is increased. We start therefore with all $R_{\max }\left(v_{\infty, k}\right)$ at zero, except that of the lowest speed in the discrete range, which is taken as the reference value. We can then find $R_{\max }$ for the next speed in the range by solving the Poisson equation numerically, imposing the condition eq. (A.39) and raising it from zero. This is then done recursively for the entire range of speeds. This procedure works because for $r>R_{\max }\left(v_{\infty, k}\right)$, the solution is the same as if $R_{\max }^{i}$ is taken to zero and at $r=R_{\max }\left(v_{\infty, k}\right)$ the derivative of $\varphi_{\text {esc }}(r)$ is continuous. This numerical solution is shown as the solid black line in figure 9 .

To go beyond the $\lambda_{D} \rightarrow 0$ limit in the regime of small charge accumulation, we can solve eq. (A.44) as a perturbation series in $\lambda_{D}$. We substitute the solutions we have obtained into the right hand side of eq. (A.44) to determine the $\mathcal{O}\left(\lambda_{D}\right)$ correction to the solution for the potential and so on. The resulting solutions are shown as the dashed lines in figure 9 , 


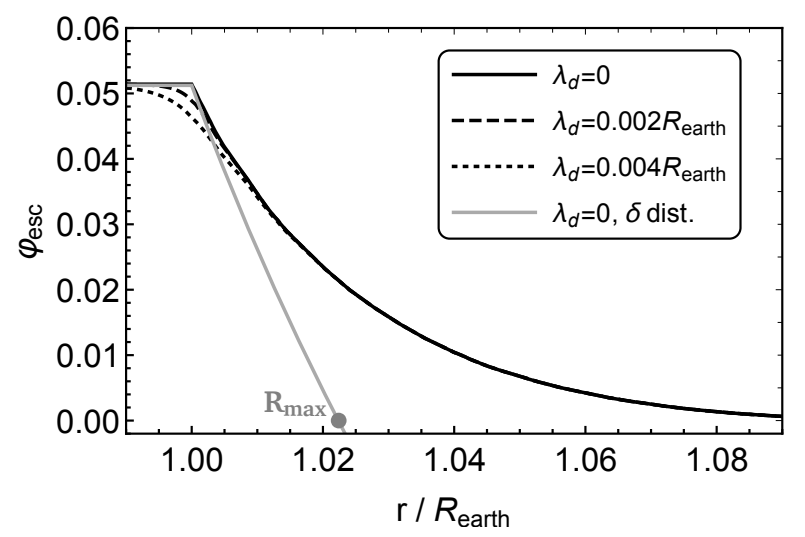

Figure 9. Mirror electric potential $\varphi_{\text {esc }}(r)$ in the disk case for $\epsilon=10^{-11}$ (the small charge accumulation regime), showing the effects of mirror helium capture inside the Earth and screening by the ambient mirror plasma. Shown are the solutions for $\varphi(r)$ for a delta function distribution and for a Maxwell-Boltzmann distribution of speeds at infinity, in the limit that the Debye length $\lambda_{D}=0$. Also shown is $\varphi_{\mathrm{esc}}(r)$ in the case of a Maxwell-Boltzmann distribution for two nonvanishing values of $\lambda_{D}$, showing how the potential is smoothed out. (The actual transition region is extremely narrow as $\lambda_{D}<10^{-3} R_{\text {Earth. }}$ )

demonstrating that the discontinuities in the first derivative of the potential are smoothed out on scales of order the Debye length at $r=R_{\text {Earth }}$ and $r=R_{\max }$.

Our careful analysis of screening effects shows that to determine capture and evaporation rates in the small charge regime, we can make use of the simple estimate,

$$
\varphi_{\mathrm{esc}}= \begin{cases}\varphi_{\mathrm{esc}}^{\text {Earth }} & \text { for } r \leq R_{\text {Earth }} \\ 0 & \text { for } r>R_{\text {Earth }}\end{cases}
$$

with $\varphi_{\text {esc }}^{\text {Earth }}$ as calculated above. We neglect the effect of the small nonvanishing potential just above the Earth's surface, which does not have a significant effect on capture or evaporation since $\left(R_{\max }-R_{\text {Earth }}\right) \ll R_{\text {Earth }}$. The mirror particle escape velocities near the surface are then modified as follows,

$$
v_{\mathrm{esc}, \hat{\mathrm{He}}}=\sqrt{v_{\mathrm{esc}}^{2}-\frac{2}{3} 2 v_{\odot, \hat{\mathrm{H} e}}^{2} \varphi_{\mathrm{esc}}^{\mathrm{Earth}}}, \quad v_{\mathrm{esc}, \hat{e}}=\sqrt{v_{\mathrm{esc}}^{2}+\frac{2}{3} v_{\odot, \hat{e}}^{2} \varphi_{\mathrm{esc}}^{\mathrm{Earth}}} .
$$

Here $v_{\text {esc }} \approx 11 \mathrm{~km} / \mathrm{s}$ is the gravitational escape velocity at the Earth's surface. This suppresses accumulation and enhances evaporation of mirror helium. For the purposes of evaporation below, any $\left(v_{\text {esc, }, \hat{\mathrm{He}}}\right)^{2}<0$ is interpreted to mean that $v_{\text {esc, }, \hat{\mathrm{He}}}=0$ at the surface, since any $\hat{H e}$ that reaches the surface immediately flies away from the Earth.

\section{A.1.4 Evaporation of mirror nuclei}

We now consider the evaporation rate of mirror helium. We conservatively assume that any $\mathrm{He}^{++}$captured inside the Earth thermalizes with the Earth's interior, which is at temperature $T_{\text {Earth }} \sim 4000 \mathrm{~K}$. Since mirror baryons typically have higher kinetic energies than this when they are captured, this can only underestimate evaporation and hence 
overestimate mirror helium accumulation. The resulting thermal velocity of mirror helium $\left\langle v_{\hat{\mathrm{He}}, \mathrm{th}}\right\rangle=\sqrt{3 T_{\mathrm{Earth}} / m_{\hat{\mathrm{He}}}} \sim 5 \mathrm{~km} / \mathrm{s}$. The virial radius corresponding to $v \sim\left\langle v_{\hat{\mathrm{He}}, \mathrm{th}}\right\rangle$ is comparable to the radius of the Earth, $R_{\text {Earth }}$. Therefore, to simplify the discussion, we assume that the captured particles are distributed homogeneously inside the Earth.

The mean free path of He in the Earth for the $\epsilon \lesssim 10^{-9}$ regime we consider can be estimated as

$$
\ell_{\hat{\mathrm{He}}} \sim \frac{1}{n_{O} \sigma_{\hat{\mathrm{H} e O}}} \approx 10^{-2} R_{\mathrm{Earth}}\left(\frac{10^{-10}}{\epsilon}\right)^{2}
$$

Here $\sigma_{\hat{H} \text { HO }}$ represents the cross section for scattering off an oxygen target, since this constitutes the most effective target that has a sizable abundance inside the Earth. The expression for $\sigma_{\hat{\mathrm{HeO}}}$ has the same form as in eq. (A.9).

When $\epsilon \lesssim 10^{-11}$, the penetration depth of mirror helium inside the Earth is comparable

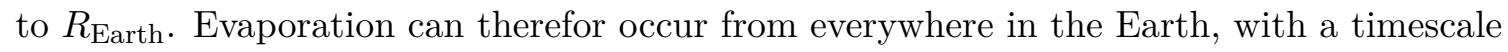
set by the mirror helium collisional time scale $\sim \ell_{\hat{\mathrm{H} e}}\left\langle v_{\hat{\mathrm{He}}, \mathrm{th}}\right\rangle^{-1}$ weighted by the probability that a mirror helium particle has enough kinetic energy to escape the earth's gravitational field. The evaporation rate is given by,

$$
n_{\hat{\mathrm{He}}}^{C} R_{e v p, \hat{\mathrm{He}}} \sim \frac{n_{\hat{\mathrm{H} e}}^{C}}{R_{\text {Earth }}^{3}} \int_{0}^{R_{\text {Earth }}} d r r^{2}\left[\left.\left\langle v_{\hat{\mathrm{He}}, \mathrm{th}}\right\rangle\right|_{T=T_{\mathrm{Earth}}(r)}\right] \ell_{\hat{\mathrm{He}}}^{-1} P_{\text {ejection }}(r) .
$$

Here we have defined an $r$-dependent ejection probability, which is simply the fraction of mirror helium nuclei in local thermal equilibrium with the Earth at temperature $T=$ $T_{\text {Earth }}(r)$ that have speeds greater than the escape velocity for helium $v_{\text {esc }}(r)$ at that location,

$$
P_{\text {ejection }}(r)=\int_{v_{\text {esc }}(r)}^{\infty} f_{M B}\left(v ; T_{\text {Earth }}(r)\right) d v
$$

where $f_{M B}$ is the Maxwell-Boltzmann distribution. We can determine $v_{\text {esc }}(r)$ from the following differential equation,

$$
\frac{1}{2} m_{\hat{\mathrm{He}}} \frac{d v^{2}}{d \tilde{r}}=-\frac{d U_{\mathrm{grav}}}{d \tilde{r}}
$$

with boundary condition $v_{f}=v\left(\tilde{r}=R_{\text {Earth }}\right)=11 \mathrm{~km} / \mathrm{s}$. Here $U_{\text {grav }}(\tilde{r})$ is the gravitational potential energy at radius $\tilde{r}$ from the center of the Earth, so the term on the right side of the equation represents the kinetic energy lost in climbing out of the Earth's potential well. In the $\epsilon \lesssim 10^{-11}$ case we are considering, the accumulated mirror electric charge is too small to generate enough electric force at the Earth's surface to eject mirror helium. This is in contrast to the $\epsilon \gtrsim 10^{-10}$ case we will study in section A.2. Since we know the temperature profile inside the Earth $T_{\text {Earth }}(r)$, this allows us to determine $P_{\text {ejection }}(r)$ as a function of $r$ and hence the evaporation rate. 


\section{A.1.5 Results}

Having determined the capture and evaporation rates, we can solve the following equations to obtain the mirror particle abundance,

$$
\begin{aligned}
\frac{d n_{\hat{\mathrm{He}}}^{C}}{d t} & =\left(R_{\text {cap, } \mathrm{He}}^{<v_{\text {cap }}}+R_{\text {cap }, \hat{\mathrm{He}}}^{>v_{\text {cap }}}\right) n_{\hat{\mathrm{He}} \mathrm{e}}^{F}-R_{\text {evp }, \hat{\mathrm{He}}} n_{\hat{\mathrm{He}} \mathrm{e}}^{C}, \quad\left(v_{\mathrm{esc}}=v_{\mathrm{esc}, \hat{\mathrm{He}}}\right) \\
n_{Q}^{C} & =2 n_{\hat{\mathrm{He}}}^{C}-n_{\hat{e}}^{C} .
\end{aligned}
$$

We can obtain number densities in the equilibrium configuration, corresponding to $d n_{\hat{\mathrm{He}}}^{C} / d t=$ $d n_{Q}^{C} / d t=0$ in eq. (A.58). As discussed in A.1.2, when $n_{Q}^{C}$ is small, the number density of captured mirror electrons is small and can be neglected. Making this assumption and setting $n_{\hat{e}}^{C}=0$, we have $n_{Q}^{C}=2 n_{\hat{\mathrm{He}}}^{C}$. Since it is the value of $n_{Q}^{C}$ that determines the capture and evaporation rates of $\hat{H e}$, this allows us to self-consistently solve for the $n_{\hat{\mathrm{He}}}^{C}$ and $n_{Q}^{C}$ that satisfy the equilibrium condition.

For a Maxwell-Boltzmann distribution of velocities with $\epsilon=10^{-11}, \hat{v} / v=3, \hat{Y}_{\odot}=1$ and local mirror baryon DM fraction $r_{\odot}=0.05$, we obtain,

$$
N_{\text {Ĥe }} \lesssim\left\{\begin{array}{l}
5 \times 10^{23}(\text { disk }) \\
\left.1 \times 10^{22} \text { (halo }\right)
\end{array}\right.
$$

and accordingly, $\varphi_{\mathrm{esc}}=0.15(0.004)$ for the disk (halo) cases. Since electrostatic shielding is only important when $\varphi_{\text {esc }} \gtrsim \mathcal{O}(1)$, its effects on the direct detection signal are negiligible. The escape velocity of mirror electrons due to the accumulated mirror helium charge is large compared to the escape velocity from gravitational effects alone, of order $\mathcal{O}(100 \mathrm{~km} / \mathrm{s})$ for both halo and disk distributions, but it is still much smaller than the thermal width of the Fermi surface in iron. Consequently evaporation of mirror electrons is highly efficient, and the number density of captured mirror electrons is small (see the discussion around eq. (A.19)). Therefore our assumption that the captured population of mirror electrons can be neglected is self-consistent. Comparing the number of captured mirror helium nuclei against eq. (A.12), we see that we are far away from the regime where self-capture becomes important.

It follows from this that for $\epsilon \lesssim 10^{-11}$, there is no significant effect on the mirror helium flux or velocity distribution in direct detection experiments, either from electrostatic or collisional shielding effects. Mirror electrons are accelerated towards the Earth by the electrostatic potential, resulting in just a slight increase in their velocity (compared to their already high ambient velocity in the plasma). This makes our sensitivity estimates for mirror electron direct detection mildly conservative.

Other choices of $\hat{v} / v$ or $\hat{Y}_{\odot}$ do not significantly change these results. This includes the limiting case when $\hat{Y}_{\odot}=0$, when the mirror baryons are entirely composed of twin hydrogen. In all cases, we find that the lowering $r_{\odot}$ results in $N_{\hat{\mathrm{He}}} \propto r_{\odot}$ (mirror baryon fraction of local DM density) because the mirror electrostatic screening is negligible. Very small $r_{\odot}$ might result in a non-negligible Debye length, but this will only modify our results by $\mathcal{O}(1)$. Therefore, capture of mirror matter has no significant effect on the direct detection signal for $\epsilon \lesssim 10^{-11}$. 


\section{A.2 $10^{-10} \lesssim \epsilon \lesssim 10^{-9}$}

We now discuss the case in which the kinetic mixing parameter $\epsilon \gtrsim 10^{-10}$. In this regime the net accumulated mirror charge density can become comparable to the ambient density, resulting in significant electrostatic effects. In addition, the potential can become large enough to suppress mirror electron evaporation, and therefore their captured fraction must be taken into account. Therefore the physics of mirror baryon accumulation in this regime is quite different from that of $\epsilon \lesssim 10^{-11}$.

\section{A.2.1 Equilibrium in the large charge regime}

For the limiting case of a delta function distribution of speeds at infinity, we obtained the Poisson equation eq. (A.44) for the dimensionless potential $\varphi_{\text {esc }}(r)$. We now consider this equation in the regime in which the net captured charge is large, so that $\beta$ can no longer be assumed to be small. Working in the limit that $\lambda_{D} \rightarrow 0$ we immediately run into the roadblock that either the potential $\varphi(r)$ must be discontinuous across the surface of the Earth, or $\varphi(r)$ is not constant inside the Earth. This is because the solution outside the Earth leads to a maximal possible value of $\varphi \simeq 0.06$ at the surface for the delta function distribution of speeds at infinity. For this limiting value of the potential inside the Earth, we can determine the captured mirror helium density that sets the right hand side of the Poisson equation to zero. Then, by integrating over the volume of the Earth we obtain the net captured charge,

$$
2 N_{\hat{\mathrm{He}}}-\left.N_{\hat{\mathrm{e}}}\right|_{\text {large q }} \approx 2 \times 10^{25} \times\left(\frac{r_{\odot}}{0.05}\right)
$$

It follows that in the $\lambda_{D} \rightarrow 0$ limit, for any value of the captured charge larger than this, either the potential must be discontinuous at $R_{\text {Earth }}$, or it must have a varying profile inside the Earth. In general, we expect that the solution in the large charge regime will exhibit both these features. This greatly complicates the calculation of the mirror electric potential.

The discussion in the paragraph above focused on the simple case in which the speed distribution far away from the Earth takes the limiting form of a delta function. However, in the $\lambda_{D} \rightarrow 0$ limit, the solution for the potential in the case of the fully realistic MaxwellBoltzmann distribution that we obtain numerically exhibits the same characteristic features, which we list below.

- For $r>R_{\text {Earth }}$ the solution of the Poisson equation sets a unique upper bound on the potential just outside the Earth's surface, which we denote as $\varphi_{\text {esc }}^{+}\left(R_{\text {Earth }}\right) \simeq 0.06(0.15)$, where the value shown is for the delta-function (Maxwell-Boltzmann) distribution.

- Just inside the Earth, there could be a sharp potential jump within a Debye length of the surface as the effects of $\beta$ "turn on". In the $\lambda_{D} \rightarrow 0$ limit, we account for this possible jump by allowing for a discontinuity in the potential at the Earth surface. We denote as $\varphi_{\text {esc }}^{-}\left(R_{\text {Earth }}\right)$ and $\varphi_{\text {esc }}^{+}\left(R_{\text {Earth }}\right)$ the potentials just inside and outside the Earth, with $\varphi_{\text {esc }}^{-}\left(R_{\text {Earth }}\right)>\varphi_{\text {esc }}^{+}\left(R_{\text {Earth }}\right)$. 
- Inside the Earth, there is some continuous potential $\varphi_{\text {esc }}(r)$ for $0 \leq r \leq R_{\text {Earth }}$ that satisfies $\varphi_{\text {esc }}\left(R_{\text {Earth }}\right)=\varphi_{\text {esc }}^{-}\left(R_{\text {Earth }}\right)$. In general the potential is expected to vary inside the Earth. However, if mirror electron capture is so efficient that their number density is much larger than the net free charge, the captured mirror electrons will distribute themselves inside the Earth in such a way that $\varphi_{\mathrm{esc}}(r)$ will tend to a constant value independent of $r$.

The numerical values of $\varphi_{\mathrm{esc}}^{+}\left(R_{\text {Earth }}\right)$ and the functional form of $\varphi_{\text {esc }}(\beta)$ exhibit a mild dependence on the precise mirror helium fraction of the dark plasma which does not affect our conclusions. They are notably independent of $r_{\odot}$ and whether we have a halo or disk distribution, since the dark baryon fraction and overall velocity drop out of the right hand side of the Poisson equation. The mirror matter fraction $r_{\odot}$ does affect the Debye length since $\lambda_{D} \sim r_{\odot}^{1 / 2}$, see eq. (A.48). If $\lambda_{D}$ is taken to be nonvanishing (but still much smaller than the size of the Earth), then the above piece-wise defined potential is smoothed out on scales of $\lambda_{D}$ near $R_{\text {Earth }}$, similar to what is shown in figure 9 . We account for effects from the nonvanishing Debye length in our discussion below, but it does not significantly change our conclusions about the general features of the potential.

A varying potential inside the Earth would necessarily result in a mirror electric force that repels the captured mirror helium particles and pushes them towards the surface. Then, to obtain the potential $\varphi_{\text {esc }}$ we would first need to solve a complicated set of coupled equations that determines the distribution of mirror helium in the Earth as a function of $\varphi$ taking into account the capture of mirror helium, its diffusion in the presence of the mirror electric field and its eventual evaporation. It follows that solving for the mirror electric potential of the Earth in the regime of significant charge accumulation is extremely challenging. However, we can make use of the fact that the potential $\varphi$ outside the Earth continues to take the same form to derive robust upper bounds on the mirror potential at equilibrium, both near the surface and deep within the Earth's interior. As we now show, this can be used to place limits on the suppression of incoming mirror helium flux near the surface where direct detection experiments are located.

\section{A.2.2 Upper bound on the net captured charge}

At equilibrium, the flux of mirror helium exiting the Earth is equal to the flux entering it. By requiring that the flux entering any region of the Earth not exceed the flux leaving it, we can derive upper bounds on $\varphi_{\text {esc }}(r)$ for $0 \leq r \leq R_{\text {Earth }}$. We begin by considering the mirror helium flux at the surface. The outgoing flux can be bounded from below under the assumption that mirror helium nuclei start out stationary just below the surface and are then accelerated by the potential jump as they leave the surface,

$$
\mathcal{F}_{\text {out }}>\mathcal{F}_{\text {out }}^{\text {min }}=n_{\hat{\mathrm{He}}}^{C} v_{\text {out }}=\left(\sum q_{i}^{2} n_{i}^{F}\right) \beta_{\hat{\mathrm{He}}}\left(R_{\text {Earth }}^{-}\right) \sqrt{\frac{4}{3}} \sqrt{\varphi_{\mathrm{esc}}^{-}\left(R_{\text {Earth }}\right)-\varphi_{\mathrm{esc}}^{+}\left(R_{\text {Earth }}\right)} v_{\odot, \hat{\mathrm{He}}},
$$

where

$$
\beta_{\hat{\mathrm{He}}}=\frac{2 e n_{\hat{\mathrm{He}}}^{C}(r)}{\sum q_{j}^{2} n_{j}^{F}}
$$


is the contribution to $\beta$ from mirror helium alone, and $v_{\text {out }}$ is the velocity of the outgoing particles. This is related to the difference between the potentials just inside and just outside the Earth, $\varphi_{\text {esc }}^{-}\left(R_{\text {Earth }}\right)-\varphi_{\text {esc }}^{+}\left(R_{\text {Earth }}\right)$, by energy conservation,

$$
v_{\text {out }}=\sqrt{\frac{4}{3}} \sqrt{\varphi_{\text {esc }}^{-}\left(R_{\text {Earth }}\right)-\varphi_{\text {esc }}^{+}\left(R_{\text {Earth }}\right)} v_{\odot, \hat{\mathrm{He}}} .
$$

This should be considered a lower bound on the outgoing flux because the electrostatic drift inside the Earth may increase the velocity of mirror nuclei just before they cross the jump. The bound is valid as long as the mean free path near the surface, eq. (A.54), is larger than the Debye length, eq. (A.48), so that collisions have no effect across the potential jump.

On the other hand, we can place an upper bound on the flux of mirror helium entering the Earth. To do this we note that the incoming flux, given by $\left\langle n_{\hat{\mathrm{He}}} v_{r}\right\rangle$, is always less than $\left\langle n_{\hat{\mathrm{He}}}|v|\right\rangle$, where the radial velocity has been replaced by the total speed. This allows us to set an upper bound on the incoming flux,

$$
\mathcal{F}_{\text {in }}<\mathcal{F}_{\text {in }}^{\max }=\int f_{\hat{H} e}\left(R_{\text {Earth }}^{+}, v\right) v d v=\left(\sum q_{i}^{2} n_{i}^{F}\right) \alpha_{\hat{\mathrm{He}}} \sqrt{\frac{2}{3 \pi}} v_{\odot, \hat{\mathrm{He}}} e^{-2 \varphi_{\mathrm{esc}}^{-}\left(R_{\mathrm{Earth}}\right)}
$$

where $f_{\hat{H e}}\left(R_{\text {Earth }}^{+}, v\right)$ is given by eq. (A.36). Note that this upper bound takes into account the full Boltzmann suppression, since for large potentials only the fastest mirror nuclei penetrate below the surface. Since the outgoing flux cannot exceed the incoming flux, $\mathcal{F}_{\text {out }} \leq \mathcal{F}_{\text {in }}$, we obtain the relation

$$
\beta_{\hat{\mathrm{He}}}\left(R_{\text {Earth }}^{-}\right) \sqrt{\frac{4}{3}} \sqrt{\varphi_{\mathrm{esc}}^{-}\left(R_{\text {Earth }}\right)-\varphi_{\mathrm{esc}}^{+}\left(R_{\text {Earth }}\right)} \leq \alpha_{\hat{\mathrm{He}}} \sqrt{\frac{2}{3 \pi}} e^{-2 \varphi_{\mathrm{esc}}^{-}\left(R_{\text {Earth }}\right)} .
$$

We now make use of the fact $\beta_{\hat{H e}}>\beta$ (we are here neglecting the effect of mirror hydrogen for simplicity, since it evaporates efficiently and its accumulation has negligible effect), which allows us to write:

$$
\beta\left(R_{\text {Earth }}^{-}\right) \sqrt{\frac{4}{3}} \sqrt{\varphi_{\text {esc }}^{-}\left(R_{\text {Earth }}\right)-\varphi_{\text {esc }}^{+}\left(R_{\text {Earth }}\right)} \leq \alpha_{\hat{\mathrm{He}}} \sqrt{\frac{2}{3 \pi}} e^{-2 \varphi_{\text {esc }}^{-}\left(R_{\text {Earth }}\right)} .
$$

For the limiting case in which the speed distribution at infinity is a delta function, eq. (A.44) allows us to relate $\varphi_{\text {esc }}^{-}$to $\beta\left(R_{\text {Earth }}\right)$ in the $\lambda_{D} \rightarrow 0$ limit. This is accomplished by setting the right hand side of eq. (A.44) to zero inside of the potential jump near the surface. This is a valid approximation in the $\lambda_{D} \rightarrow 0$ limit since the potential does not rapidly change on Debye length scales once we are past the potential jump near the Earth's surface. This allows us to determine $\beta\left(R_{\text {Earth }}^{-}\right)$, just inside of the potential jump, as a function of $\varphi_{\text {esc }}^{-}\left(R_{\text {Earth }}\right)$. With this, eq. (A.66) can be translated into an upper bound on the potential just inside the Earth,

$$
\varphi_{\text {esc }}^{-}\left(R_{\text {Earth }}\right)<0.6(0.35)
$$

calculated using the Maxwell-Boltzmann (delta-function) distribution at infinity. Notice that $v_{\odot, \hat{H e}}$ dropped out of eq. (A.66), which means that this upper bound applies to both halo and disk distributions. The resulting electrostatic suppression of the incoming mirror 
helium flux for direct detection experiments near the surface can be obtained by considering the radial flux,

$$
\mathcal{F}_{i}(r)=\int d v \frac{r_{\infty}^{2}}{r^{2}} \frac{d v_{\infty}}{d v} f_{i}\left(r_{\infty}, v_{\infty}\right) \int v_{r} \frac{v_{r, \infty}}{v_{r}} \gamma\left(v_{\infty}, v_{r, \infty}, r_{\infty}, r\right) \frac{d v_{r, \infty}}{v_{\infty}}
$$

evaluated at $r=R_{\text {Earth }}$. This suppression factor turns out to be $\operatorname{simply} \min \left(e^{-2 \varphi_{\text {esc }}^{-}\left(R_{\text {Earth }}\right)}, 1\right)$ $\simeq 0.3$ for the Maxwell-Boltzmann distribution of speeds at infinity. While we can neglect the effects of captured mirror hydrogen, any incoming mirror hydrogen flux would nonetheless also be affected by this electrostatic screening. The corresponding suppression is $\min \left(e^{-\varphi_{\text {esc }}^{-}\left(R_{\text {Earth }}\right)}, 1\right) \simeq 0.55$ (halo). The effect of this suppression on the direct detection bounds for $\epsilon$ is marginal — less than $50 \%$ (25\%) for mirror helium (hydrogen) searches. Our sensitivity projections for $\epsilon \sqrt{r_{\odot}}$ from mirror nuclear recoils will therefore have at most a factor of 2 uncertainty, but only if $r_{\odot} \ll 0.01$ is so small that the sensitivity boundary lies near or above $\epsilon \sim 10^{-10}$. For $r_{\odot} \sim 0.01$, we find that future experiments probe kinetic mixings that are far below $10^{-10}$, meaning the projected sensitivities are not affected by this modest suppression. In our discussion to this point we have neglected the effects of the Earth's gravity, which acts to slightly reduce in the extent of electrostatic shielding. The resulting correction to the results is modest in the disk case and negligible in the halo case.

We move to find a similar bound on $\varphi_{\text {esc }}$ anywhere inside the Earth. As we will see, this can be used to place a limit on the net number of captured particles inside the Earth, and thereby show that collisional shielding does not limit direct detection. A non-uniform mirror-electric potential $\varphi_{\mathrm{esc}}(r)$ inside the Earth initiates a drift velocity towards the surface,

$$
v_{\text {drift }} \sim a \Delta t_{\text {coll }},
$$

where $\Delta t_{\text {coll }}$ denotes the average time between collisions, and $a$ represents the radial acceleration of mirror helium charges due to the electric field. The value of $\Delta t_{\text {coll }}$ is determined by the mirror helium mean free path inside the Earth, eq. (A.54), and the average velocity of mirror helium nuclei inside the Earth. The latter is dominated either by the drift velocity itself or by the thermal contribution that arises from the mirror nuclei coming into equilibrium with the Earth's interior. To approximately account for the limits where either contribution could dominate, we write

$$
\Delta t_{\mathrm{coll}} \sim \frac{\ell_{\hat{\mathrm{He}}}}{v_{\mathrm{drift}}+v_{\hat{\mathrm{He}}, \mathrm{th}}},
$$

which yields,

$$
\begin{aligned}
& v_{\text {drift }} \sim \sqrt{a \ell_{\hat{\mathrm{He}}}+\left(\frac{v_{\hat{\mathrm{He}}, \mathrm{th}}}{2}\right)^{2}}-\left(\frac{v_{\hat{\mathrm{He}}, \mathrm{th}}}{2}\right) \\
& =\sqrt{2 \varphi_{\mathrm{esc}}^{\prime}(r) \ell_{\hat{\mathrm{He}}}\left(\frac{v_{\odot, \hat{\mathrm{He}}}}{\sqrt{3}}\right)^{2}+\left(\frac{v_{\hat{\mathrm{He}}, \mathrm{th}}}{2}\right)^{2}}-\left(\frac{v_{\hat{\mathrm{He}}, \mathrm{th}}}{2}\right) \text {, }
\end{aligned}
$$

where we have taken

$$
a=\frac{2}{3} \varphi_{\mathrm{esc}}^{\prime}(r) v_{\odot, \hat{\mathrm{He}}}^{2}
$$




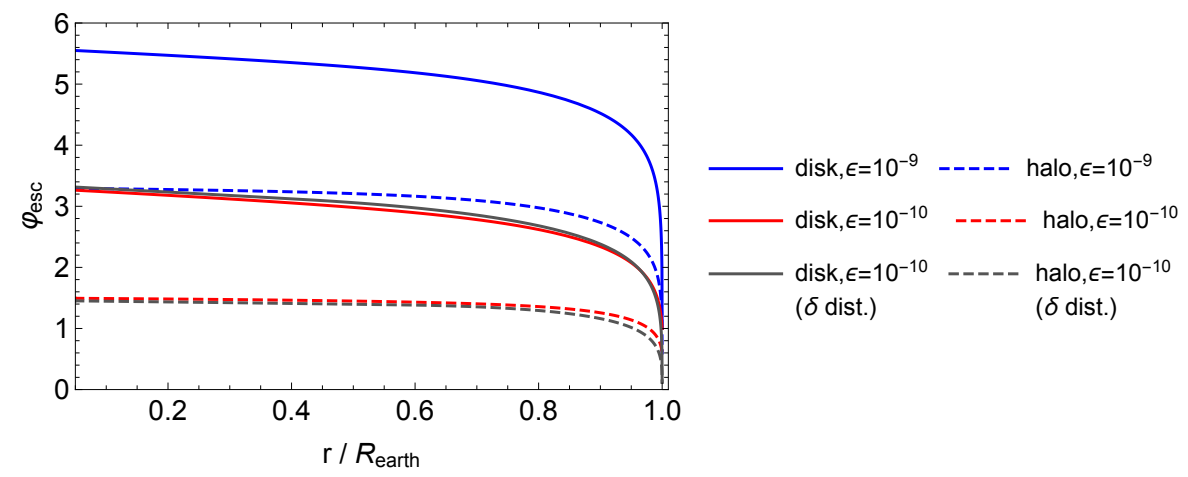

Figure 10. Maximum possible mirror electric potential inside the Earth, parametrized in terms of $\varphi_{\text {esc }}(r)$, shown for mirror particles in a halo (solid) and disk (dashed) distribution. Results are shown for a delta function distribution of speeds at infinity with $\epsilon=10^{-10}$ (grey) and for a Maxwell-Boltzmann distribution of speeds at infinity with $\epsilon=10^{-10}$ (red) and $\epsilon=10^{-9}$ (blue).

The outgoing radial flux must be less than the incoming radial flux at any arbitrary location $r<R_{\text {Earth. }}$. As in eq. (A.64), we overestimate the incoming flux by replacing the radial component of the velocity by the total speed. From this we obtain the inequality,

$$
\beta(r) v_{\text {drift }} \leq \beta_{\hat{\mathrm{He}}}(r) v_{\mathrm{drift}} \leq \alpha_{\hat{\mathrm{He}}} \sqrt{\frac{2}{3 \pi}} v_{\odot, \hat{\mathrm{He}}} e^{-2 \varphi_{\mathrm{esc}}(r)} .
$$

In the case of a delta function distribution of velocities we can relate $\beta(r)$ to $\varphi_{\text {esc }}(r)$ by setting the right hand side of eq. (A.44) to zero. We can obtain the corresponding relation for the case of a Maxwell-Boltzmann distribution from our numerical solution. Saturating the above inequality yields a differential equation for the maximum possible mirror electric potential $\varphi_{\mathrm{esc}}^{\max }(r)$. This differential equation can be solved numerically. The solution is shown in figure 10 for the delta function and Maxwell-Boltzmann distributions of velocities, for both halo and disk profiles. The effects of gravity, although subdominant, have been included. From the corresponding $\beta(r)$, we can obtain an upper bound on the net captured charge by integrating over the volume of the Earth. For $\epsilon \lesssim 10^{-9}$ we obtain,

$$
2 N_{\hat{\mathrm{He}}}-N_{\hat{\mathrm{e}}}<0.5 \sum q_{i}^{2} n_{i}^{F} \frac{4}{3} \pi R_{\text {Earth }}^{3} \approx 3(8) \times 10^{25} \times\left(\frac{r_{\odot}}{0.05}\right),
$$

for the Maxwell-Boltzmann (delta-function) distribution of speeds at infinity. This bound is almost identical for the halo and disk profiles, since the lower repulsive potential in the disk case is compensated for by the smaller incoming flux.

If $r_{\odot} \lesssim 10^{-7}\left(10^{-5}\right)$ in disk (halo) distributions, $\lambda_{D}$ becomes comparable to or larger than the mirror helium mean free path $\ell_{\hat{\mathrm{He}}}$. In this parameter range the results we obtained above are slightly modified. The potential jump just below the Earth's surface is now smoothed out on scales of $\lambda_{D} \gtrsim 100 \mathrm{~km}$, and we have to take collisions into account when computing the outgoing flux near the surface. The collisions suppress the outgoing velocity for the same potential difference by a factor of $\mathcal{O}\left(\ell_{\hat{\mathrm{He}}} / \lambda_{D}\right)$. However, we see that the potential is only logarithmically sensitive to this due to the exponential dependence of the incoming flux on $\varphi_{\text {esc }}$ in eq. (A.64). Therefore, although this effect increases the 
upper bound on the potential 'inside the jump' in the Earth's interior by a factor of $\sim \log \left(\lambda_{D} / \ell_{\hat{\mathrm{He}}}\right)$ compared to eq. (A.67), the potential at the location of direct detection experiments at depth $d \sim \mathcal{O}(\mathrm{km})$ below the Earth's surface is actually smaller by a factor of $\sim d / \lambda_{D}$ since the potential varies smoothly on distance scales of order the Debye length instead of sharply increasing at the surface. This means that the effective maximum $\varphi_{\text {esc }}$ that electrostatically shields direct detection experiments is reduced by a factor of $\sim\left(d / \lambda_{D}\right) \log \left(\lambda_{D} / \ell_{\hat{\mathrm{He}}}\right)$. Lowering $r_{\text {all }}$ therefore further reduces the modest electrostatic suppression of the incoming mirror helium flux.

\section{A.2.3 Upper bound on the total number of captured mirror particles}

The constraint calculated in eq. (A.74) represents an upper bound on the net charge of the captured mirror particles, $2 N_{\hat{\mathrm{He}}}-N_{\hat{e}}$. In order to establish that the effects of collisional shielding are not important, we need to obtain upper bounds on $N_{\hat{\mathrm{He}}}$ and $N_{\hat{e}}$ separately. We do this by noting that if the number of captured mirror electrons is much larger than the net captured charge, so that $N_{\hat{e}} \gg 2 N_{\hat{\mathrm{He}}}-N_{\hat{e}}$, the mirror electrons will move freely inside the Earth to neutralize any gradients in the electric field. Therefore, in this limit the value of the potential $\varphi_{\text {esc }}$ takes on the same value at every point in the Earth ${ }^{17}$. This constant value is necessarily less than or equal to the upper bounds on $\varphi_{\text {esc }}^{-}\left(R_{\text {Earth }}\right)$ shown in eq. (A.67) for the halo and disk cases.

We now show that this behavior can be used to set a bound on the neutral component that complements the bound on the net captured charge in eq. (A.74). In section A.1.2 we obtained an upper bound on the net number of captured mirror electrons that depends on the potential $\varphi_{\text {esc }}$ as,

$$
N_{\hat{e}} \lesssim 10^{23} \exp \left(\varphi_{\mathrm{esc}} \frac{T_{\text {mirror }}}{T_{e}}\right)
$$

for the disc (halo) case. Setting $\varphi_{\text {esc }}$ in this equation to its upper bound in eq. (A.67) we find $N_{\hat{e}} \lesssim 10^{24}$ for both the disk and halo cases. This upper bound on $N_{\hat{e}}$ has been obtained under the assumption that the number of captured mirror electrons is much larger than the net captured charge, $N_{\hat{e}} \gg 2 N_{\hat{\mathrm{He}}}-N_{\hat{e}}$. If this assumption is valid, the upper bound on $N_{\hat{e}}$ also translates into an upper bound on the number of captured helium nuclei, $N_{\hat{H e}} \ll N_{\hat{e}}$. It follows that the number of captured mirror particles is at most of order $10^{24}$, far too small to result in significant self-capture.

If the assumption that the number of captured mirror electrons is much larger than the net captured charge is not valid, so that $N_{\hat{e}} \lesssim 2 N_{\hat{H} \text { e }}-N_{\hat{e}}$, we can nevertheless still obtain an upper bound on the captured population. In this case the upper bounds on the net captured charge in eq. (A.74) translate into upper bounds on the $N_{\hat{e}}$ and $N_{\hat{\mathrm{He}}}$ individually, so that $N_{\hat{e}}, N_{\hat{H e}} \lesssim 10^{26}$. These are still far below the numbers required for self-capture to play a major role. We therefore conclude that self-capture and collisional shielding do not significantly affect direct detection.

\footnotetext{
${ }^{17}$ This can be seen by solving the Debye-Huckel equation for $\varphi_{\mathrm{esc}}$ inside the Earth.
} 
Open Access. This article is distributed under the terms of the Creative Commons Attribution License (CC-BY 4.0), which permits any use, distribution and reproduction in any medium, provided the original author(s) and source are credited.

\section{References}

[1] Z. Chacko, H.-S. Goh and R. Harnik, The Twin Higgs: Natural electroweak breaking from mirror symmetry, Phys. Rev. Lett. 96 (2006) 231802 [hep-ph/0506256] [INSPIRE].

[2] R. Barbieri, T. Gregoire and L.J. Hall, Mirror world at the large hadron collider, hep-ph/0509242 [INSPIRE].

[3] Z. Chacko, Y. Nomura, M. Papucci and G. Perez, Natural little hierarchy from a partially goldstone twin Higgs, JHEP 01 (2006) 126 [hep-ph/0510273] [INSPIRE].

[4] A. Falkowski, S. Pokorski and M. Schmaltz, Twin SUSY, Phys. Rev. D 74 (2006) 035003 [hep-ph/0604066] [INSPIRE].

[5] S. Chang, L.J. Hall and N. Weiner, A Supersymmetric twin Higgs, Phys. Rev. D 75 (2007) 035009 [hep-ph/0604076] [INSPIRE].

[6] N. Craig and K. Howe, Doubling down on naturalness with a supersymmetric twin Higgs, JHEP 03 (2014) 140 [arXiv:1312.1341] [INSPIRE].

[7] A. Katz, A. Mariotti, S. Pokorski, D. Redigolo and R. Ziegler, SUSY Meets Her Twin, JHEP 01 (2017) 142 [arXiv: 1611.08615] [INSPIRE].

[8] M. Badziak and K. Harigaya, Supersymmetric D-term Twin Higgs, JHEP 06 (2017) 065 [arXiv: 1703.02122] [INSPIRE].

[9] M. Badziak and K. Harigaya, Minimal Non-Abelian Supersymmetric Twin Higgs, JHEP 10 (2017) 109 [arXiv: 1707.09071] [INSPIRE].

[10] M. Badziak and K. Harigaya, Asymptotically Free Natural Supersymmetric Twin Higgs Model, Phys. Rev. Lett. 120 (2018) 211803 [arXiv:1711.11040] [InSPIRE].

[11] M. Geller and O. Telem, Holographic Twin Higgs Model, Phys. Rev. Lett. 114 (2015) 191801 [arXiv: 1411.2974] [INSPIRE].

[12] R. Barbieri, D. Greco, R. Rattazzi and A. Wulzer, The Composite Twin Higgs scenario, JHEP 08 (2015) 161 [arXiv:1501.07803] [INSPIRE].

[13] M. Low, A. Tesi and L.-T. Wang, Twin Higgs mechanism and a composite Higgs boson, Phys. Rev. D 91 (2015) 095012 [arXiv: 1501.07890] [INSPIRE].

[14] ATLAS collaboration, Search for a scalar partner of the top quark in the jets plus missing transverse momentum final state at $\sqrt{s}=13 \mathrm{TeV}$ with the ATLAS detector, JHEP 12 (2017) 085 [arXiv: 1709.04183] [INSPIRE].

[15] ATLAS collaboration, Search for direct top squark pair production in final states with two leptons in $\sqrt{s}=13 \mathrm{TeV}$ pp collisions with the ATLAS detector, Eur. Phys. J. C 77 (2017) 898 [arXiv: 1708.03247] [INSPIRE].

[16] ATLAS collaboration, Search for direct top squark pair production in events with a Higgs or $Z$ boson, and missing transverse momentum in $\sqrt{s}=13 \mathrm{TeV}$ pp collisions with the ATLAS detector, JHEP 08 (2017) 006 [arXiv: 1706.03986] [INSPIRE]. 
[17] ATLAS collaboration, Search for new phenomena in final states with an energetic jet and large missing transverse momentum in pp collisions at $\sqrt{s}=13 \mathrm{TeV}$ using the ATLAS detector, Phys. Rev. D 94 (2016) 032005 [arXiv: 1604.07773] [INSPIRE].

[18] CMS collaboration, Search for supersymmetry in proton-proton collisions at $13 \mathrm{TeV}$ using identified top quarks, Phys. Rev. D 97 (2018) 012007 [arXiv:1710.11188] [InSPIRE].

[19] CMS collaboration, Search for supersymmetry in multijet events with missing transverse momentum in proton-proton collisions at 13 TeV, Phys. Rev. D 96 (2017) 032003 [arXiv: 1704.07781] [INSPIRE].

[20] CMS collaboration, Search for supersymmetry in the all-hadronic final state using top quark tagging in pp collisions at $\sqrt{s}=13 \mathrm{TeV}$, Phys. Rev. D 96 (2017) 012004 [arXiv:1701.01954] [INSPIRE].

[21] R. Foot, H. Lew and R.R. Volkas, Possible consequences of parity conservation, Mod. Phys. Lett. A 7 (1992) 2567 [INSPIRE].

[22] G. Burdman, Z. Chacko, R. Harnik, L. de Lima and C.B. Verhaaren, Colorless Top Partners, a $125 \mathrm{GeV}$ Higgs, and the Limits on Naturalness, Phys. Rev. D 91 (2015) 055007 [arXiv:1411.3310] [INSPIRE].

[23] R. Harnik, K. Howe and J. Kearney, Tadpole-Induced Electroweak Symmetry Breaking and PNGB Higgs Models, JHEP 03 (2017) 111 [arXiv: 1603.03772] [INSPIRE].

[24] H. Beauchesne, K. Earl and T. Grégoire, The spontaneous $\mathbb{Z}_{2}$ breaking Twin Higgs, JHEP 01 (2016) 130 [arXiv:1510.06069] [INSPIRE].

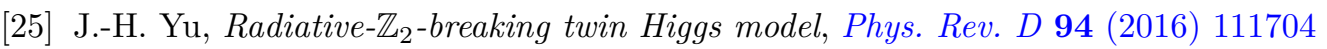
[arXiv: 1608.01314] [INSPIRE].

[26] J.-H. Yu, A tale of twin Higgs: natural twin two Higgs doublet models, JHEP 12 (2016) 143 [arXiv: 1608.05713] [INSPIRE].

[27] B. Batell and C.B. Verhaaren, Breaking Mirror Twin Hypercharge, JHEP 12 (2019) 010 [arXiv: 1904.10468] [INSPIRE].

[28] B. Batell, W. Hu and C.B. Verhaaren, Breaking Mirror Twin Color, JHEP 08 (2020) 009 [arXiv: 2004.10761] [INSPIRE].

[29] C. Kilic and S. Swaminathan, Can A Pseudo-Nambu-Goldstone Higgs Lead To Symmetry Non-Restoration?, JHEP 01 (2016) 002 [arXiv:1508.05121] [INSPIRE].

[30] K. Fujikura, K. Kamada, Y. Nakai and M. Yamaguchi, Phase Transitions in Twin Higgs Models, JHEP 12 (2018) 018 [arXiv:1810.00574] [INSPIRE].

[31] Z. Chacko, N. Craig, P.J. Fox and R. Harnik, Cosmology in Mirror Twin Higgs and Neutrino Masses, JHEP 07 (2017) 023 [arXiv: 1611.07975] [INSPIRE].

[32] N. Craig, S. Koren and T. Trott, Cosmological Signals of a Mirror Twin Higgs, JHEP 05 (2017) 038 [arXiv: 1611.07977] [INSPIRE].

[33] Planck collaboration, Planck 2018 results. VI. Cosmological parameters, Astron. Astrophys. 641 (2020) A6 [Erratum ibid. 652 (2021) C4] [arXiv: 1807. 06209] [INSPIRE].

[34] A.G. Riess et al., New Parallaxes of Galactic Cepheids from Spatially Scanning the Hubble Space Telescope: Implications for the Hubble Constant, Astrophys. J. 855 (2018) 136 [arXiv: 1801.01120] [INSPIRE]. 
[35] M. Farina, Asymmetric Twin Dark Matter, JCAP 11 (2015) 017 [arXiv:1506.03520] [INSPIRE].

[36] R. Barbieri, L.J. Hall and K. Harigaya, Minimal Mirror Twin Higgs, JHEP 11 (2016) 172 [arXiv: 1609.05589] [INSPIRE].

[37] C. Csáki, E. Kuflik and S. Lombardo, Viable Twin Cosmology from Neutrino Mixing, Phys. Rev. D 96 (2017) 055013 [arXiv: 1703.06884] [InSPIRE].

[38] R. Barbieri, L.J. Hall and K. Harigaya, Effective Theory of Flavor for Minimal Mirror Twin Higgs, JHEP 10 (2017) 015 [arXiv:1706. 05548] [INSPIRE].

[39] F. Bishara and C.B. Verhaaren, Singleton Portals to the Twin Sector, JHEP 05 (2019) 016 [arXiv: 1811.05977] [INSPIRE].

[40] D. Liu and N. Weiner, A Portalino to the Twin Sector, arXiv:1905.00861 [INSPIRE].

[41] K. Harigaya, R. Mcgehee, H. Murayama and K. Schutz, A predictive mirror twin Higgs with small $Z_{2}$ breaking, JHEP 05 (2020) 155 [arXiv: 1905.08798] [INSPIRE].

[42] N. Craig, S. Knapen, P. Longhi and M. Strassler, The Vector-like Twin Higgs, JHEP 07 (2016) 002 [arXiv: 1601.07181] [INSPIRE].

[43] N. Craig, A. Katz, M. Strassler and R. Sundrum, Naturalness in the Dark at the LHC, JHEP 07 (2015) 105 [arXiv: 1501.05310] [INSPIRE].

[44] D. Curtin and C.B. Verhaaren, Discovering Uncolored Naturalness in Exotic Higgs Decays, JHEP 12 (2015) 072 [arXiv: 1506.06141] [INSPIRE].

[45] C. Csáki, E. Kuflik, S. Lombardo and O. Slone, Searching for displaced Higgs boson decays, Phys. Rev. D 92 (2015) 073008 [arXiv: 1508.01522] [INSPIRE].

[46] C. Kilic, S. Najjari and C.B. Verhaaren, Discovering the Twin Higgs Boson with Displaced Decays, Phys. Rev. D 99 (2019) 075029 [arXiv:1812.08173] [InSPIRE].

[47] S. Alipour-Fard, N. Craig, S. Gori, S. Koren and D. Redigolo, The second Higgs at the lifetime frontier, JHEP 07 (2020) 029 [arXiv: 1812.09315] [INSPIRE].

[48] Z.G. Berezhiani and R.N. Mohapatra, Reconciling present neutrino puzzles: Sterile neutrinos as mirror neutrinos, Phys. Rev. D 52 (1995) 6607 [hep-ph/9505385] [INSPIRE].

[49] Z.G. Berezhiani, A.D. Dolgov and R.N. Mohapatra, Asymmetric inflationary reheating and the nature of mirror universe, Phys. Lett. B 375 (1996) 26 [hep-ph/9511221] [INSPIRE].

[50] P. Adshead, Y. Cui and J. Shelton, Chilly Dark Sectors and Asymmetric Reheating, JHEP 06 (2016) 016 [arXiv: 1604.02458] [INSPIRE].

[51] N. Craig and A. Katz, The Fraternal WIMP Miracle, JCAP 10 (2015) 054 [arXiv: 1505. 07113] [INSPIRE].

[52] I. Garcia Garcia, R. Lasenby and J. March-Russell, Twin Higgs WIMP Dark Matter, Phys. Rev. D 92 (2015) 055034 [arXiv: 1505.07109] [InSPIRE].

[53] I. Garcia Garcia, R. Lasenby and J. March-Russell, Twin Higgs Asymmetric Dark Matter, Phys. Rev. Lett. 115 (2015) 121801 [arXiv:1505.07410] [INSPIRE].

[54] M. Farina, A. Monteux and C.S. Shin, Twin mechanism for baryon and dark matter asymmetries, Phys. Rev. D 94 (2016) 035017 [arXiv:1604.08211] [INSPIRE].

[55] V. Prilepina and Y. Tsai, Reconciling Large And Small-Scale Structure In Twin Higgs Models, JHEP 09 (2017) 033 [arXiv:1611.05879] [INSPIRE]. 
[56] Y. Hochberg, E. Kuflik and H. Murayama, Twin Higgs model with strongly interacting massive particle dark matter, Phys. Rev. D 99 (2019) 015005 [arXiv: 1805. 09345] [INSPIRE].

[57] H.-C. Cheng, L. Li and R. Zheng, Coscattering/Coannihilation Dark Matter in a Fraternal Twin Higgs Model, JHEP 09 (2018) 098 [arXiv: 1805.12139] [INSPIRE].

[58] J. Terning, C.B. Verhaaren and K. Zora, Composite Twin Dark Matter, Phys. Rev. D 99 (2019) 095020 [arXiv: 1902.08211] [INSPIRE].

[59] S. Koren and R. McGehee, Freezing-in twin dark matter, Phys. Rev. D 101 (2020) 055024 [arXiv: 1908.03559] [INSPIRE].

[60] M. Badziak, G. Grilli Di Cortona and K. Harigaya, Natural Twin Neutralino Dark Matter, Phys. Rev. Lett. 124 (2020) 121803 [arXiv:1911.03481] [InSPIRE].

[61] W.-Z. Feng and J.-H. Yu, Twin Cogenesis, arXiv:2005.06471 [INSPIRE].

[62] Z. Chacko, D. Curtin, M. Geller and Y. Tsai, Cosmological Signatures of a Mirror Twin Higgs, JHEP 09 (2018) 163 [arXiv:1803.03263] [INSPIRE].

[63] H. Vogel and J. Redondo, Dark Radiation constraints on minicharged particles in models with a hidden photon, JCAP 02 (2014) 029 [arXiv: 1311.2600] [INSPIRE].

[64] T. Gherghetta, J. Kersten, K. Olive and M. Pospelov, Evaluating the price of tiny kinetic mixing, Phys. Rev. D 100 (2019) 095001 [arXiv:1909.00696] [INSPIRE].

[65] D. Curtin and J. Setford, Direct Detection of Atomic Dark Matter in White Dwarfs, JHEP 03 (2021) 166 [arXiv: 2010.00601] [INSPIRE].

[66] D. Curtin and J. Setford, How To Discover Mirror Stars, Phys. Lett. B 804 (2020) 135391 [arXiv: 1909.04071] [INSPIRE].

[67] D. Curtin and J. Setford, Signatures of Mirror Stars, JHEP 03 (2020) 041 [arXiv: 1909.04072] [INSPIRE].

[68] H. Winch, J. Setford, J. Bovy and D. Curtin, Using LSST Microlensing to Constrain Dark Compact Objects in Spherical and Disk Configurations, arXiv:2012.07136 [INSPIRE].

[69] M. Hippert, J. Setford, H. Tan, D. Curtin, J. Noronha-Hostler and N. Yunes, Mirror Neutron Stars, arXiv:2103.01965 [INSPIRE].

[70] R.N. Mohapatra and V.L. Teplitz, Structures in the mirror universe, Astrophys. J. 478 (1997) 29 [astro-ph/9603049] [INSPIRE].

[71] R.N. Mohapatra and V.L. Teplitz, Mirror dark matter and galaxy core densities of galaxies, Phys. Rev. D 62 (2000) 063506 [astro-ph/0001362] [INSPIRE].

[72] J.-S. Roux and J.M. Cline, Constraining galactic structures of mirror dark matter, Phys. Rev. D 102 (2020) 063518 [arXiv:2001.11504] [INSPIRE].

[73] R. Foot, Implications of the DAMA and CRESST experiments for mirror matter type dark matter, Phys. Rev. D 69 (2004) 036001 [hep-ph/0308254] [INSPIRE].

[74] H. An, S.-L. Chen, R.N. Mohapatra, S. Nussinov and Y. Zhang, Energy Dependence of Direct Detection Cross Section for Asymmetric Mirror Dark Matter, Phys. Rev. D 82 (2010) 023533 [arXiv: 1004.3296] [INSPIRE].

[75] R. Foot, A comprehensive analysis of the dark matter direct detection experiments in the mirror dark matter framework, Phys. Rev. D 82 (2010) 095001 [arXiv: 1008. 0685] [INSPIRE].

[76] A. Addazi et al., DAMA annual modulation effect and asymmetric mirror matter, Eur. Phys. J. C 75 (2015) 400 [arXiv: 1507.04317] [INSPIRE]. 
[77] J.D. Clarke and R. Foot, Mirror dark matter will be confirmed or excluded by XENON1T, Phys. Lett. B 766 (2017) 29 [arXiv:1606.09063] [INSPIRE].

[78] Z. Berezhiani, Mirror world and its cosmological consequences, Int. J. Mod. Phys. A 19 (2004) 3775 [hep-ph/0312335] [INSPIRE].

[79] L.B. Okun, Mirror particles and mirror matter: 50 years of speculations and search, Phys. Usp. 50 (2007) 380 [hep-ph/0606202] [INSPIRE].

[80] P. Ciarcelluti, Cosmology with mirror dark matter, Int. J. Mod. Phys. D 19 (2010) 2151 [arXiv:1102.5530] [INSPIRE].

[81] R. Foot, Mirror dark matter: Cosmology, galaxy structure and direct detection, Int. J. Mod. Phys. A 29 (2014) 1430013 [arXiv:1401.3965] [inSPIRE].

[82] H. Goldberg and L.J. Hall, A New Candidate for Dark Matter, Phys. Lett. B 174 (1986) 151 [INSPIRE].

[83] D.E. Kaplan, G.Z. Krnjaic, K.R. Rehermann and C.M. Wells, Atomic Dark Matter, JCAP 05 (2010) 021 [arXiv:0909.0753] [INSPIRE].

[84] D.E. Kaplan, G.Z. Krnjaic, K.R. Rehermann and C.M. Wells, Dark Atoms: Asymmetry and Direct Detection, JCAP 10 (2011) 011 [arXiv:1105.2073] [INSPIRE].

[85] J.M. Cline, Z. Liu and W. Xue, Millicharged Atomic Dark Matter, Phys. Rev. D 85 (2012) 101302 [arXiv: 1201.4858 ] [INSPIRE].

[86] J.M. Cline, Z. Liu, G. Moore and W. Xue, Scattering properties of dark atoms and molecules, Phys. Rev. D 89 (2014) 043514 [arXiv:1311.6468] [INSPIRE].

[87] J. Fan, A. Katz, L. Randall and M. Reece, Double-Disk Dark Matter, Phys. Dark Univ. 2 (2013) 139 [arXiv:1303.1521] [InSPIRE].

[88] J. Fan, A. Katz, L. Randall and M. Reece, Dark-Disk Universe, Phys. Rev. Lett. 110 (2013) 211302 [arXiv: 1303.3271] [INSPIRE].

[89] J. Fan, A. Katz and J. Shelton, Direct and indirect detection of dissipative dark matter, JCAP 06 (2014) 059 [arXiv: 1312.1336] [INSPIRE].

[90] F.-Y. Cyr-Racine, R. de Putter, A. Raccanelli and K. Sigurdson, Constraints on Large-Scale Dark Acoustic Oscillations from Cosmology, Phys. Rev. D 89 (2014) 063517 [arXiv: 1310.3278] [INSPIRE].

[91] E. Rosenberg and J. Fan, Cooling in a Dissipative Dark Sector, Phys. Rev. D 96 (2017) 123001 [arXiv: 1705.10341] [INSPIRE].

[92] A. Ghalsasi and M. McQuinn, Exploring the astrophysics of dark atoms, Phys. Rev. D 97 (2018) 123018 [arXiv:1712.04779] [INSPIRE].

[93] M.I. Gresham, H.K. Lou and K.M. Zurek, Astrophysical Signatures of Asymmetric Dark Matter Bound States, Phys. Rev. D 98 (2018) 096001 [arXiv: 1805.04512] [InSPIRE].

[94] R. Essig, S.D. Mcdermott, H.-B. Yu and Y.-M. Zhong, Constraining Dissipative Dark Matter Self-Interactions, Phys. Rev. Lett. 123 (2019) 121102 [arXiv: 1809.01144] [INSPIRE].

[95] G. Alvarez and H.-B. Yu, Astrophysical probes of inelastic dark matter with a light mediator, Phys. Rev. D 101 (2020) 043002 [arXiv:1911.11114] [InSPIRE].

[96] K.M. Zurek, Asymmetric Dark Matter: Theories, Signatures, and Constraints, Phys. Rept. 537 (2014) 91 [arXiv: 1308.0338] [INSPIRE]. 
[97] K. Petraki and R.R. Volkas, Review of asymmetric dark matter, Int. J. Mod. Phys. A 28 (2013) 1330028 [arXiv:1305.4939] [INSPIRE].

[98] H. Mo, F.C. van den Bosch and S. White, Galaxy Formation and Evolution, Cambridge University Press (2010).

[99] F. Nesti and P. Salucci, The Dark Matter halo of the Milky Way, AD 2013, JCAP 07 (2013) 016 [arXiv: 1304.5127] [INSPIRE].

[100] J.K. Werk et al., The COS-Halos Survey: Physical Conditions and Baryonic Mass in the Low-Redshift Circumgalactic Medium, Astrophys. J. 792 (2014) 8 [arXiv:1403.0947] [INSPIRE].

[101] E.D. Kramer and L. Randall, Updated Kinematic Constraints on a Dark Disk, Astrophys. J. 824 (2016) 116 [arXiv: 1604.01407] [InSPIRE].

[102] E.D. Kramer and L. Randall, Interstellar Gas and a Dark Disk, Astrophys. J. 829 (2016) 126 [arXiv: 1603.03058] [INSPIRE].

[103] P.F. Hopkins et al., Galaxies on FIRE (Feedback In Realistic Environments): Stellar Feedback Explains Cosmologically Inefficient Star Formation, Mon. Not. Roy. Astron. Soc. 445 (2014) 581 [arXiv: 1311.2073] [INSPIRE].

[104] P.F. Hopkins et al., How To Model Supernovae in Simulations of Star and Galaxy Formation, Mon. Not. Roy. Astron. Soc. 477 (2018) 1578 [arXiv: 1707.07010] [inSPIRE].

[105] P.F. Hopkins et al., FIRE-2 Simulations: Physics versus Numerics in Galaxy Formation, Mon. Not. Roy. Astron. Soc. 480 (2018) 800 [arXiv:1702.06148] [inSPIRE].

[106] F. Governato et al., The Romulus cosmological simulations: a physical approach to the formation, dynamics and accretion models of SMBHs, Mon. Not. Roy. Astron. Soc. 470 (2017) 1121.

[107] M. Tremmel et al., Introducing romulusc: a cosmological simulation of a galaxy cluster with an unprecedented resolution, Mon. Not. Roy. Astron. Soc. 483 (2018) 3336.

[108] M. Vogelsberger et al., Properties of galaxies reproduced by a hydrodynamic simulation, Nature 509 (2014) 177 [arXiv: 1405.1418] [INSPIRE].

[109] M. Vogelsberger et al., Introducing the Illustris Project: Simulating the coevolution of dark and visible matter in the Universe, Mon. Not. Roy. Astron. Soc. 444 (2014) 1518 [arXiv: 1405.2921] [INSPIRE].

[110] S. Genel et al., Introducing the Illustris Project: the evolution of galaxy populations across cosmic time, Mon. Not. Roy. Astron. Soc. 445 (2014) 175 [arXiv:1405.3749] [INSPIRE].

[111] D. Sijacki et al., The Illustris simulation: the evolving population of black holes across cosmic time, Mon. Not. Roy. Astron. Soc. 452 (2015) 575 [arXiv: 1408.6842] [INSPIRE].

[112] J. Schaye et al., The EAGLE project: Simulating the evolution and assembly of galaxies and their environments, Mon. Not. Roy. Astron. Soc. 446 (2015) 521 [arXiv:1407.7040] [INSPIRE].

[113] M. Schaller et al., The EAGLE simulations of galaxy formation: the importance of the hydrodynamics scheme, Mon. Not. Roy. Astron. Soc. 454 (2015) 2277 [arXiv:1509.05056] [INSPIRE].

[114] M. Schaller et al., Baryon effects on the internal structure of $\Lambda$ CDM haloes in the EAGLE simulations, Mon. Not. Roy. Astron. Soc. 451 (2015) 1247 [arXiv:1409.8617] [INSPIRE]. 
[115] O. Pols, Stellar Structure and Evolution, https://www.astro.ru.nl/ onnop/education/stev_utrecht_notes/.

[116] L. Randall and M. Reece, Dark Matter as a Trigger for Periodic Comet Impacts, Phys. Rev. Lett. 112 (2014) 161301 [arXiv: 1403.0576] [INSPIRE].

[117] L. Randall and J. Scholtz, Dissipative Dark Matter and the Andromeda Plane of Satellites, JCAP 09 (2015) 057 [arXiv: 1412.1839] [InSPIRE].

[118] R. Foot and Z.K. Silagadze, Thin disk of co-rotating dwarfs: A fingerprint of dissipative (mirror) dark matter?, Phys. Dark Univ. 2 (2013) 163 [arXiv:1306.1305] [InSPIRE].

[119] P. Agrawal and L. Randall, Point Sources from Dissipative Dark Matter, JCAP 12 (2017) 019 [arXiv: 1706.04195] [INSPIRE].

[120] L. Spitzer Jr., The Dynamics of the Interstellar Medium. I. Local Equilibrium, Astrophys. J. 93 (1941) 369.

[121] G.B. Rybicki and A.P. Lightman, Radiative processes in astrophysics, Wiley-Interscience Publication (1979).

[122] N. Straumann, General relativity and relativistic astrophysics, Springer (1984).

[123] R.N. Mohapatra and V.L. Teplitz, Mirror matter MACHOs, Phys. Lett. B 462 (1999) 302 [astro-ph/9902085] [INSPIRE].

[124] R. Foot, Have mirror stars been observed?, Phys. Lett. B 452 (1999) 83 [astro-ph/9902065] [INSPIRE].

[125] R. Foot, A.Y. Ignatiev and R.R. Volkas, Physics of mirror photons, Phys. Lett. B 503 (2001) 355 [astro-ph/0011156] [inSPIRE].

[126] Z. Berezhiani, S. Cassisi, P. Ciarcelluti and A. Pietrinferni, Evolutionary and structural properties of mirror star MACHOs, Astropart. Phys. 24 (2006) 495 [astro-ph/0507153] [INSPIRE].

[127] E. Michaely, I. Goldman and S. Nussinov, Solar luminosity bounds on mirror matter, Phys. Rev. D 101 (2020) 123006 [arXiv:1905.12643] [INSPIRE].

[128] R. Foot, Mirror dark matter interpretation of the DAMA/Libra annual modulation signal, AIP Conf. Proc. 1178 (2009) 111 [arXiv:0907.0048] [inSPIRE].

[129] R. Huo, H.-B. Yu and Y.-M. Zhong, The Structure of Dissipative Dark Matter Halos, JCAP 06 (2020) 051 [arXiv: 1912.06757] [INSPIRE].

[130] I.S. Butsky and T.R. Quinn, The Role of Cosmic-ray Transport in Shaping the Simulated Circumgalactic Medium, Astrophys. J. 868 (2018) 108 [arXiv:1803.06345].

[131] W. Dehnen and J. Binney, Local stellar kinematics from hipparcos data, Mon. Not. Roy. Astron. Soc. 298 (1998) 387 [astro-ph/9710077] [INSPIRE].

[132] M.C. Smith et al., The RAVE Survey: Constraining the Local Galactic Escape Speed, Mon. Not. Roy. Astron. Soc. 379 (2007) 755 [astro-ph/0611671] [INSPIRE].

[133] J. Lopez-Santiago, D. Montes, I. Crespo-Chacon and M.J. Fernandez-Figueroa, The nearest young moving groups, Astrophys. J. 643 (2006) 1160 [astro-ph/0601573] [INSPIRE].

[134] K. Schutz, T. Lin, B.R. Safdi and C.-L. Wu, Constraining a Thin Dark Matter Disk with Gaia, Phys. Rev. Lett. 121 (2018) 081101 [arXiv:1711. 03103] [INSPIRE].

[135] J. Buch, S.C.J. Leung and J. Fan, Using Gaia DR2 to Constrain Local Dark Matter Density and Thin Dark Disk, JCAP 04 (2019) 026 [arXiv:1808.05603] [INSPIRE]. 
[136] J. Pollack, D.N. Spergel and P.J. Steinhardt, Supermassive Black Holes from Ultra-Strongly Self-Interacting Dark Matter, Astrophys. J. 804 (2015) 131 [arXiv:1501.00017] [INSPIRE].

[137] J. Choquette, J.M. Cline and J.M. Cornell, Early formation of supermassive black holes via dark matter self-interactions, JCAP 07 (2019) 036 [arXiv: 1812.05088] [INSPIRE].

[138] G. D'Amico, P. Panci, A. Lupi, S. Bovino and J. Silk, Massive Black Holes from Dissipative Dark Matter, Mon. Not. Roy. Astron. Soc. 473 (2018) 328 [arXiv:1707.03419] [INSPIRE].

[139] R. Essig et al., Working Group Report: New Light Weakly Coupled Particles, in Community Summer Study 2013: Snowmass on the Mississippi, (2013) [arXiv:1311.0029] [InSPIRE].

[140] L. Chuzhoy and E.W. Kolb, Reopening the window on charged dark matter, JCAP 07 (2009) 014 [arXiv:0809.0436] [INSPIRE].

[141] S.D. McDermott, H.-B. Yu and K.M. Zurek, Turning off the Lights: How Dark is Dark Matter?, Phys. Rev. D 83 (2011) 063509 [arXiv:1011.2907] [InSPIRE].

[142] R. Foot, Do magnetic fields prevent mirror particles from entering the galactic disk?, Phys. Lett. B 699 (2011) 230 [arXiv:1011.5078] [INSPIRE].

[143] G. D'Amico, M. Kamionkowski and K. Sigurdson, Dark Matter Astrophysics, arXiv:0907.1912 [INSPIRE].

[144] R. Foot, Shielding of a direct detection experiment and implications for the DAMA annual modulation signal, Phys. Lett. B 789 (2019) 592 [arXiv: 1806.04293] [INSPIRE].

[145] J.D. Lewin and P.F. Smith, Review of mathematics, numerical factors, and corrections for dark matter experiments based on elastic nuclear recoil, Astropart. Phys. 6 (1996) 87 [INSPIRE].

[146] R. Essig, T. Volansky and T.-T. Yu, New Constraints and Prospects for sub-GeV Dark Matter Scattering off Electrons in Xenon, Phys. Rev. D 96 (2017) 043017 [arXiv: 1703.00910] [INSPIRE].

[147] LUX collaboration, First results from the LUX dark matter experiment at the Sanford Underground Research Facility, Phys. Rev. Lett. 112 (2014) 091303 [arXiv:1310. 8214] [INSPIRE].

[148] R. Essig, M. Fernandez-Serra, J. Mardon, A. Soto, T. Volansky and T.-T. Yu, Direct Detection of sub-GeV Dark Matter with Semiconductor Targets, JHEP 05 (2016) 046 [arXiv: 1509.01598] [INSPIRE].

[149] SuperCDMS collaboration, Projected Sensitivity of the SuperCDMS SNOLAB experiment, Phys. Rev. D 95 (2017) 082002 [arXiv:1610.00006] [INSPIRE].

[150] SENSEI collaboration, Single-electron and single-photon sensitivity with a silicon Skipper CCD, Phys. Rev. Lett. 119 (2017) 131802 [arXiv:1706. 00028] [InSPIRE].

[151] Y. Hochberg, M. Pyle, Y. Zhao and K.M. Zurek, Detecting Superlight Dark Matter with Fermi-Degenerate Materials, JHEP 08 (2016) 057 [arXiv:1512.04533] [InSPIRE].

[152] Y. Hochberg, Y. Kahn, M. Lisanti, C.G. Tully and K.M. Zurek, Directional detection of dark matter with two-dimensional targets, Phys. Lett. B 772 (2017) 239 [arXiv:1606.08849] [INSPIRE].

[153] R. Essig, J. Mardon and T. Volansky, Direct Detection of Sub-GeV Dark Matter, Phys. Rev. D 85 (2012) 076007 [arXiv: 1108.5383] [inSPIRE]. 
[154] R. Essig, A. Manalaysay, J. Mardon, P. Sorensen and T. Volansky, First Direct Detection Limits on sub-GeV Dark Matter from XENON10, Phys. Rev. Lett. 109 (2012) 021301 [arXiv:1206.2644] [INSPIRE].

[155] S.K. Lee, M. Lisanti, S. Mishra-Sharma and B.R. Safdi, Modulation Effects in Dark Matter-Electron Scattering Experiments, Phys. Rev. D 92 (2015) 083517 [arXiv:1508.07361] [INSPIRE].

[156] P.W. Graham, D.E. Kaplan, S. Rajendran and M.T. Walters, Semiconductor Probes of Light Dark Matter, Phys. Dark Univ. 1 (2012) 32 [arXiv:1203.2531] [INSPIRE].

[157] S. Derenzo, R. Essig, A. Massari, A. Soto and T.-T. Yu, Direct Detection of sub-GeV Dark Matter with Scintillating Targets, Phys. Rev. D 96 (2017) 016026 [arXiv:1607.01009] [INSPIRE].

[158] Y. Hochberg, Y. Zhao and K.M. Zurek, Superconducting Detectors for Superlight Dark Matter, Phys. Rev. Lett. 116 (2016) 011301 [arXiv:1504.07237] [INSPIRE].

[159] XENON10 collaboration, A search for light dark matter in XENON10 data, Phys. Rev. Lett. 107 (2011) 051301 [Erratum ibid. 110 (2013) 249901] [arXiv: 1104.3088] [INSPIRE].

[160] LUX collaboration, First direct detection constraint on mirror dark matter kinetic mixing using LUX 2013 data, Phys. Rev. D 101 (2020) 012003 [arXiv: 1908.03479] [INSPIRE].

[161] XENON collaboration, Light Dark Matter Search with Ionization Signals in XENON1T, Phys. Rev. Lett. 123 (2019) 251801 [arXiv: 1907.11485] [InSPIRE].

[162] SENSEI collaboration, SENSEI: Direct-Detection Constraints on Sub-GeV Dark Matter from a Shallow Underground Run Using a Prototype Skipper-CCD, Phys. Rev. Lett. 122 (2019) 161801 [arXiv: 1901.10478] [INSPIRE].

[163] S. Betts et al., Development of a Relic Neutrino Detection Experiment at PTOLEMY: Princeton Tritium Observatory for Light, Early-Universe, Massive-Neutrino Yield, in Community Summer Study 2013: Snowmass on the Mississippi, (2013) [arXiv:1307.4738] [INSPIRE].

[164] Y. Hochberg, I. Charaev, S.-W. Nam, V. Verma, M. Colangelo and K.K. Berggren, Detecting Sub-GeV Dark Matter with Superconducting Nanowires, Phys. Rev. Lett. 123 (2019) 151802 [arXiv: 1903.05101] [INSPIRE].

[165] S. Knapen, T. Lin, M. Pyle and K.M. Zurek, Detection of Light Dark Matter With Optical Phonons in Polar Materials, Phys. Lett. B 785 (2018) 386 [arXiv:1712.06598] [InSPIRE].

[166] S. Griffin, S. Knapen, T. Lin and K.M. Zurek, Directional Detection of Light Dark Matter with Polar Materials, Phys. Rev. D 98 (2018) 115034 [arXiv:1807.10291] [inSPIRE].

[167] XENON collaboration, Excess electronic recoil events in XENON1T, Phys. Rev. D 102 (2020) 072004 [arXiv:2006.09721] [INSPIRE].

[168] L. Zu, G.-W. Yuan, L. Feng and Y.-Z. Fan, Mirror Dark Matter and Electronic Recoil Events in XENON1T, Nucl. Phys. B 965 (2021) 115369 [arXiv:2006.14577] [INSPIRE].

[169] N. Ashcroft and N. Mermin, Solid State Physics, Saunders College, Philadelphia (1976). 